\title{
MANCHESTER
}

1824

\section{An Indirect Measurement of the Width of the W Boson at the DØ Experiment}

\author{
Paul Telford \\ 2006 \\ Particle Physics Group \\ School of Physics and Astronomy
}

A thesis submitted to The University of Manchester for the degree of Doctor of Philosophy in the Faculty of Engineering and Physical Sciences 


\section{Contents}

2.1 The Standard Model . . . . . . . . . . . . . . . . . . . . 21

2.1.1 Fundamental Particles . . . . . . . . . . . . . . 22

2.1.2 Quantum Field Theories ............... 24

2.1.3 The Electroweak Interaction . . . . . . . . . . . . 25

2.1 .4 The Strong Interaction . . . . . . . . . . . . . . . . . . . 29

2.2 Current Directions in Particle Physics . . . . . . . . . . . . . 30

2.2.1 Recent and Future Experiments . . . . . . . . . . . . 30

2.2.2 Current Avenues of Research . . . . . . . . . . . . . . 33

3 Experimental Apparatus 38

3.1 The Fermilab Tevatron . . . . . . . . . . . . . . . . . 39

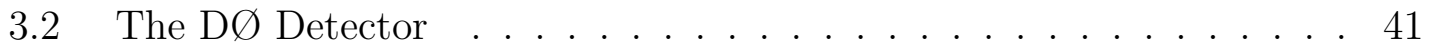

3.2.1 The D $\varnothing$ Co-ordinate System . . . . . . . . . . . . . 42 
3.2.2 The Central Tracking System . . . . . . . . . . . . . . 43

3.2.3 The Calorimeter . . . . . . . . . . . . . . . . 47

3.2.4 The Muon Detectors ................ . 51

3.2.5 The Luminosity Monitors . . . . . . . . . . . . . . . 54

3.3 The D $\varnothing$ Trigger System . . . . . . . . . . . . . . . 54

3.3.1 The Level 1 Trigger . . . . . . . . . . . . . . 55

3.3.2 The Level 2 Trigger . . . . . . . . . . . . . . 55

3.3.3 The Level 3 Trigger . . . . . . . . . . . . . . . 56

3.4 Event Reconstruction at D . . . . . . . . . . . . . . . 56

3.4.1 Central Track Reconstruction ............. 57

3.4.2 Vertex and Beamspot Reconstruction . . . . . . . . . . 57

3.4.3 Calorimeter Object Reconstruction . . . . . . . . . . . 58

3.4.4 Muon Reconstruction ............... 58

3.4.5 Detection of Neutrinos . . . . . . . . . . . . 59

4 Simulation of Events using Monte Carlo Generators $\quad 61$

4.1 Event Generators . . . . . . . . . . . . . . . . 62

4.2 Detector Simulation . . . . . . . . . . . . . . 63

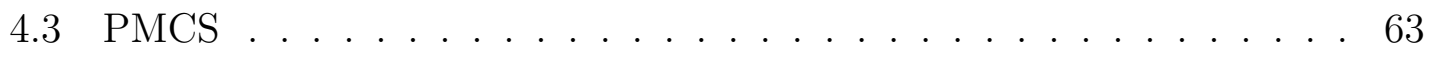

4.3.1 Track Resolution ... . . . . . . . . . . . . 64

4.3.2 Addition of Tracking Efficiencies . . . . . . . . . . . . . 65

4.3.3 Muon Resolution and Efficiencies . . . . . . . . . . . . 67 
4.3.4 Electron, Photon and Jet Resolution . . . . . . . . . . . . 68

4.3.5 Missing Energy Resolution . . . . . . . . . . . . . . 70

4.3.6 Simulation of Triggers . . . . . . . . . . . . . . 72

5 Electroweak Physics at the Tevatron $\quad 74$

5.1 Production and Decay Mechanisms . . . . . . . . . . . 75

5.1.1 Invariant Mass Distribution of the Z Boson . . . . . . . . . . . 76

5.1 .2 Particle Widths . . . . . . . . . . . . . . 77

5.1.3 Transverse Mass Distribution of the W Boson . . . . . . . . . 78

5.2 Measurements using Electroweak Bosons . . . . . . . . . . . . . . 79

5.2.1 Properties of Electroweak Bosons . . . . . . . . . . . . . 79

5.2.2 Electroweak Bosons as Calibration Tools . . . . . . . . . . . 80

5.3 Indirect Measurement of the W Boson Width . . . . . . . . . . . . . 82

6 Measurement of $\mathrm{W} \rightarrow \mu \nu$ and $\mathrm{Z} / \gamma^{*} \rightarrow \mu^{+} \mu^{-}$Cross Sections $\quad 83$

6.1 Event Selections . . . . . . . . . . . . . . . . 84

6.1.1 $\boldsymbol{Z} / \boldsymbol{\gamma}^{*} \rightarrow \boldsymbol{\mu}^{+} \boldsymbol{\mu}^{-}$Event Selection . . . . . . . . . . . 85

6.1.2 $\mathrm{W} \rightarrow \mu \nu$ Event Selection . . . . . . . . . . . . . 87

6.2 Measurement of Efficiencies . . . . . . . . . . . . . . 88

6.2.1 $\mathrm{Z} / \boldsymbol{\gamma}^{*} \rightarrow \boldsymbol{\mu}^{+} \boldsymbol{\mu}^{-}$Efficiencies .............. . . 89

$6.2 .2 \quad \mathbf{W} \rightarrow \boldsymbol{\mu \nu}$ Efficiencies . . . . . . . . . . . . . . 91

6.3 Estimation of Background . . . . . . . . . . . . . . . . 91 
6.3.1 Backgrounds to the $\mathrm{Z} / \gamma^{*} \rightarrow \mu^{+} \mu^{-}$Events . . . . . . . . . . . 92

6.3.2 Backgrounds to $\boldsymbol{W} \rightarrow \boldsymbol{\mu \nu}$ Events . . . . . . . . . . . . 94

6.4 Integrated Luminosity . . . . . . . . . . . . . . . . . 97

6.5 Summary of Results . . . . . . . . . . . . . . . . . . . . 98

7 Parton Distribution Functions 100

7.1 Theoretical Background . . . . . . . . . . . . . . . 100

7.2 The CTEQ6 PDF Sets . . . . . . . . . . . . . . . . . 102

7.3 Uncertainties on PDFs . . . . . . . . . . . . . . . . 104

7.4 Uncertainties on Observables . . . . . . . . . . . . . . 105

8 Studies for the PMCS Simulation 109

8.1 Modelling Track Resolution _. . . . . . . . . . . . . . . . . . 109

8.1.1 Studies using using $\boldsymbol{Z} \rightarrow \boldsymbol{\mu}^{+} \boldsymbol{\mu}^{-}$Events . . . . . . . . . . 110

8.1.2 Studies Using $\mathbf{J} / \boldsymbol{\Psi} \rightarrow \boldsymbol{\mu}^{+} \boldsymbol{\mu}^{-}$Events . . . . . . . . . . . 114

8.1.3 Summary of the Tuned Parameters . . . . . . . . . . . 119

8.2 Modelling of Efficiencies . . . . . . . . . . . . . . . . . . 120

8.2.1 Tracking Efficiencies . . . . . . . . . . . . . 121

8.2.2 Muon Efficiencies . . . . . . . . . . . . . . . 121

8.2.3 Trigger efficiencies ................ . . 123

8.2.4 Uncertainty on Observables . . . . . . . . . . . . . . 128

8.3 Applications of PMCS . . . . . . . . . . . . . . . . . . . . . . 128 
8.3.1 Efficiency of Z/ $\gamma^{*} \rightarrow \mu^{+} \mu^{-}$Event Selection . . . . . . . . . . . 129

8.3.2 Other Analyses using PMCS .............. 144

9 Indirect Measurement of the Width of the W Boson 148

9.1 The Drell-Yan Correction . . . . . . . . . . . . . . . . . . 148

9.1.1 Derivation of the Drell-Yan Correction. . . . . . . . . . . . . . 149

9.1.2 Calculation of the Drell Yan Correction . . . . . . . . . . . . . 149

9.2 Measurement of $\boldsymbol{R}$. . . . . . . . . . . . . . . . . . 150

9.2.1 Correlated Uncertainties ................ 151

9.2.2 Determination of $R$. . . . . . . . . . . . . . . 157

9.3 Width of the W Boson from $R$. . . . . . . . . . . . . . 158

9.4 Discussion .......................... 160

10 Conclusions

A Normalising Backgrounds

A.1 Normalising to the Signal . . . . . . . . . . . . . . . . . 173

A.2 Normalising to the Total Number of Events . . . . . . . . . . . . . . 174

B Definition of the Octant Boundaries

B.1 The Central Region (The wamus) . . . . . . . . . . . 175

B.2 The Forward Region (The FAmus) . . . . . . . . . . . . . . . 176

C Old Form of the Drell Yan Correction

Word count: 35,000 words 


\section{List of Figures}

2.1 Feynman diagrams showing the process $\mathrm{e}^{+} \mathrm{e}^{-} \rightarrow \mu^{+} \mu^{-}$. The left hand diagram shows this at tree level, the right hand diagram shows this with one of the first order corrections. . . . . . . . . . . . . . . . . . 27

2.2 The mass of the $\mathrm{W}$ boson, $M_{W}$, plotted against the mass of the top quark, $M_{t}$. The yellow band shows the allowed range of values for the Standard Model Higgs boson. The green ovoid shows the constraints placed upon $M_{W}$ and $M_{t}$ by direct measurement. The red ellipse shows the constraints placed upon these values by indirect measurements [10] . . . . . . . . . . 37

3.1 Drawing of the Fermilab accelerator chain. . . . . . . . . . . . . . 39

3.2 Cross section of the $\mathrm{D} \varnothing$ detector . . . . . . . . . . . . . 41

3.3 The $\mathrm{D} \varnothing$ detector tracking system . . . . . . . . . . . . . . . 43

3.4 The SMT . . . . . . . . . . . . . . . . . . . . . . . . . 45

3.5 Diagram illustrating the mounting of the fibres on the carbon cylinders within the CFT. The radial doublet structure can be clearly seen. . . . . . . . . . . . . . . . . 47

3.6 A segmented view of a quarter of the calorimeter. . . . . . . . . . . 50

3.7 The muon system $[23] . \ldots \ldots \ldots$. . . . . . . . . . . . . . 52 
4.1 Diagram of tag and probe method used to evaluate tracking efficiency. 66

5.1 Diagram of $\mathrm{Z}$ boson production from quark-antiquark fusion. . . . . . 75

5.2 Invariant mass distribution of events passing the $\mathrm{Z} \rightarrow \mu^{+} \mu^{-}$event selection [35]. . . . . . . . . . . . . . . . . . . . . . 77

6.1 Isolation rate, in single muon events, as a function of the $p_{t}$ of the muon. The different bands represent different cuts on $\not_{T}$ and $m_{T}$ [42]. 95

7.1 Distribution of $\frac{1}{x}$ and $Q$ values used in global fit used to produce the CTEQ5 PDFs $[51] . \ldots \ldots 102$

7.2 Fractional difference between each of the 40 error sets and the central PDF for the $\mathrm{Z} \rightarrow \mu^{+} \mu^{-}$cross section (top plot) and $R_{\sigma}$. The 'up' error sets are shaded blue and the 'down' error sets are shaded magenta. . 106

$8.1 \chi^{2}$ comparison of $\mathrm{Z} \rightarrow \mu^{+} \mu^{-}$peak between data and PMCS with varying values of $A$ in the top plot and $C$ in the bottom plot . . . . . . 113

$8.2 \chi^{2}$ comparison of $\mathrm{Z} \rightarrow \mu^{+} \mu^{-}$peak between data, with the $\mathrm{W} \rightarrow \mu \nu$ track quality cuts applied, and PMCS with varying values of $A$ in the top plot and $C$ in the bottom plot . . . . . . . . . . . 115

8.3 $\mathrm{J} / \Psi \rightarrow \mu^{+} \mu^{-}$peak fitted with Gaussian plus first order Polynomial. . 116

8.4 Variation of by the fitted mean of the $\mathrm{J} / \Psi \rightarrow \mu^{+} \mu^{-}$peak in PMCS with a) $B$ and b) $\frac{1}{C}$. . . . . . . . . . . . . . . 117

8.5 Variation of by the fitted width of the $\mathrm{J} / \Psi \rightarrow \mu^{+} \mu^{-}$peak in PMCS with a) $B$ and b) $\frac{1}{C}$. . . . . . . . . . . . . . . . 118 
8.6 Cross-check to ensure that the tracking efficiency is correctly added into PMCS. This is shown, as a function of $\eta_{c f t}$, for tracks in the region $-10<z<10 \mathrm{~cm}$. The line are the input efficiencies measured from data and the points with error bars is the output efficiency measured from PMCS. . . . . . . . . . . . . . . . . . . . . . . . 122

8.7 The distribution of the muon $\eta_{A}$ for the three different trigger combinations. The data is shown as the points with error bars. The PMCS prediction is shown as blue histograms. . . . . . . . . . . 127

8.8 The spread in values of $R$ as evaluated in Section 9.2.1. . . . . . . . 129

8.9 Di-muon invariant mass distribution for all three data taking periods combined. The data is the points with error bars. The blue histogram is the PMCS prediction with yellow bands denoting the syst-stat uncertainty. The contribution from backgrounds is shown as the filled red histogram. . . . . . . . . . . . . . . . . . . 131

8.10 The distribution of the muon $p_{t}$ with all selection cuts except the $p_{t}$ cut on the muon with the lower amount of $p_{t}$ in the event. The data is shown as the points with error bars. The PMCS prediction is shown as blue histograms with yellow bands for the syst-stat uncertainty. The upper histogram shows this for the muon in the event with the higher $p_{t}$. The lower plot shows this for the muon in the event with the lower $p_{t}$. . . . . . . . . . . . . . . . . . . . . . . . . . . . . . . . . 132

8.11 The distribution of the muon $\eta_{A}$. The data is shown as the points with error bars. The PMCS prediction is shown as blue histograms with yellow bands for the syst-stat uncertainty. The upper histogram shows this for the first data taking period, the lower for the last data taking period. . . . . . . . . . . . . . . . . 134 
8.12 The distribution of the muon $\phi_{A}$. The data is shown as the points with error bars. The PMCs prediction is shown as blue histograms with yellow bands for the syst-stat uncertainty. The upper histogram shows this for the first data taking period, the lower for the last data taking period. . . . . . . . . . . . . . . . 135

8.13 The distribution of the $\mathrm{Z}$ boson $p_{t}$. The data is shown as the points with error bars. The PMCs prediction is shown as blue histograms with yellow bands for the syst-stat uncertainty. The upper histogram shows this on a standard scale, the lower on a logarithmic scale. . . . 136

8.14 The distribution of the $\mathrm{Z}$ boson $p_{t}$. The data is shown as the points with error bars. The PMCs prediction is shown as blue histograms with yellow bands for the syst-stat uncertainty. The upper histogram shows this on a standard scale, the lower on a logarithmic scale. . . . 137

8.15 Ratio of number of events in data and PMCS, as a function of $z$. The PMCS sample is generated using a single Gaussian, with width of $26.7 \mathrm{~cm}$, and no corrections applied. The total number of events in PMCS was normalised to the total number of events in data. . . . . 139

8.16 Distribution of the $z$ vertex of the tracks passing the events selection. The top plot shows this on a standard scale, the bottom on a log scale. The data is shown as the points with error bars. The PMCS predictions are shown using a black histogram for the central value and a blue histogram for the Gaussian, width $28 \mathrm{~cm}$. . . . . . . . . . . . . . . . . . 140

8.17 Comparison between of the transverse mass distribution of $W \gamma$ pairs in data (points) and PMCS(black line). The contribution from background processes is represented by the grey histogram [64] . . . . . . 145

8.18 Comparison of $\eta$ distribution in data (red points) and PMCs (black line) for events attributed to diffractive $\mathrm{Z} \rightarrow \mu^{+} \mu^{-}$production. . . . . 146 
8.19 Comparison between data and parameterised Monte Carlo. The blue circles are data, the red line is $\mathrm{Z} / \gamma^{*} \rightarrow \mu^{+} \mu^{-}$Monte Carlo. The signal expected to be observed for different Planck scales are shown [68]. . . 147

9.1 The Standard Model Prediction for the width of the $\mathrm{W}$ boson. The experimental measurements from D $\varnothing$ and CDF Run II are shown as points with error bars. The PDG average of all other experimental results is shown as a point with an error bar. . . . . . . . . . . 162 


\section{List of Tables}

2.1 List of fermions in the Standard Model, each with mass and electric charge. The left hand column indicates the generation $(G)$. All numbers are obtained from $[2] \ldots \ldots \ldots$

2.2 List of fundamental gauge mediating bosons in the Standard Model. All numbers are obtained from Reference $[2] \ldots \ldots$. . . . . . . . 24

4.1 Parameters used in local muon $p_{t}$ resolution. . . . . . . . . . . 67

4.2 Summary of parameters used to smear $E_{T} \ldots \ldots \ldots \ldots$

5.1 Branching fractions of the decays of the $\mathrm{W}$ boson $[2] \ldots \ldots 75$

5.2 Branching fractions of the decays of the $\mathrm{Z}$ boson $[2] \ldots \ldots 76$

6.1 Summary of efficiencies in the $\mathrm{Z} / \gamma^{*} \rightarrow \mu^{+} \mu^{-}$analysis [35]. $\ldots \ldots 90$

6.2 Summary of efficiencies in the $\mathrm{W} \rightarrow \mu \nu$ analysis [44]. . . . . . 91

6.3 Summary of backgrounds to the $\mathrm{Z} / \gamma^{*} \rightarrow \mu^{+} \mu^{-}$analysis. . . . . . . 94

6.4 Summary of backgrounds to the $\mathrm{W} \rightarrow \mu \nu$ analysis [42] $\ldots \ldots . .97$

6.5 Summary of numbers used to produce the cross sections. . . . . . . 99 
7.1 PDF Uncertainty on the $\mathrm{Z} / \gamma^{*} \rightarrow \mu^{+} \mu^{-}$cross section and $R_{\sigma}$ using the prescriptions described above. . . . . . . . . . . . . . 108

8.1 Parameters used in track resolution. . . . . . . . . . . . . . . 119

8.2 Co-ordinates used to input efficiencies into PMCS. L2 denotes all Level 2 and Level 1 wires efficiencies. The 'muon' efficiency is the combined medium, L1scint, L1wires and L2M3 efficiency used in the $\mathrm{W} \rightarrow \mu \nu$ analysis. . . . . . . . . . . . . . . 120

8.3 $\epsilon_{\mathrm{MC}}$ as evaluated for the three data taking periods of the $\mathrm{Z} / \gamma^{*} \rightarrow \mu^{+} \mu^{-}$ analysis. The statistical uncertainty is negligible. The systematic uncertainty is evaluated in detail in Section 8.3.1 . . . . . . . . . 130

8.4 Summary of systematic uncertainties on $\epsilon_{\mathrm{MC}} \ldots \ldots$. . . . . . . . . . 141

8.5 Breakdown of the acceptance measured using HERWIG and PYTHIA. The numbers are consecutive so that the bottom hole efficiency is for those events that pass the cuts in $x_{A}$ and $y_{A}$. The cuts are defined in Section 6.1. . . . . . . . . . . . . . . . . . . . . . . . . 142

8.6 Trigger efficiencies measured using the independent trigger method and using PMCs. . . . . . . . . . . . . . . . . . . . . 143

9.1 Values of the cross sections used to calculate the Drell Yan correction. 150

9.2 Summary of PDF Uncertainties using three different prescriptions. . . 152

9.3 Summary of syst-stat Uncertainties with correlations properly accounted for and ignored. . . . . . . . . . . . . . . . 154

9.4 Summary of the numbers used to calculate $R$. . . . . . . . . . 158

9.5 Summary of the uncertainties due to the statistical limitations on the number of candidate events. . . . . . . . . . . . . . 158 
9.6 Summary of the uncertainties on the backgrounds. . . . . . . . . . . 159

9.7 Summary of the uncertainties due to uncertainties on the acceptances. 159

B.1 Octant Boundaries in the Central Region. The table lists the distance, in $\phi$, from the edge of a given region to the centre of the octant boundary.176 


\begin{abstract}
This thesis presents an indirect measurement of the width of the $\mathrm{W}$ boson using data collected at the $\mathrm{D} \varnothing$ experiment, a multipurpose particle detector utilising the Fermilab Tevatron. The $\mathrm{W}$ width was determined from the ratio of $\mathrm{W} \rightarrow \mu \nu$ to $\mathrm{Z} \rightarrow \mu^{+} \mu^{-}$cross sections to be

$$
\Gamma_{W}=2168 \pm 22(\text { stat }) \pm 62(\text { syst })_{-16}^{+24}(\text { pdf }) \pm 4(\text { other }) \mathrm{MeV}
$$

in good agreement with the Standard Model prediction and other experimental measurements.

In addition there is a description of how work made towards this measurement has been used to improve the parameterised detector simulation, a vital tool in the obtention of physics results from signals observed in the detector, and in estimating the uncertainty due to choice of PDF, which is of interest for all measurements made at hadron colliders.
\end{abstract}


No portion of the work referred to in this thesis has been submitted in support of an application for another degree or qualification of this or any other university or other institute of learning.

Copyright in text of this thesis rests with the author. Copies (by any process) either in full, or of extracts, may be made only in accordance with instructions given by the Author and lodged in the John Rylands University Library of Manchester. Details may be obtained from the librarian. This page must form part of any such copies made. Further copies (by any process) of copies made in accordance with such instructions may not be made without the permission (in writing) of the Author.

The ownership of any intellectual property rights which may be described in this thesis is vested in the University of Manchester, subject to any prior agreement to the contrary, and may not be made available for use by third parties without the written permission of the University, which will prescribe the terms and conditions of any such agreement.

Further information on the conditions under which disclosures and exploitation may take place is available from the Head of the School of Physics and Astronomy.

This work was supported financially by the Particle Physics and Astronomy Research Council (PPARC) between October 2002 and September 2005. 


\section{Acknowledgments}

The collaborative nature of particle physics means that I owe the completion of this to many people, too many to mention here. First I would like to thank my supervisor, Terry Wyatt, for his enthusiasm and support. It would be impossible to forget to thank Stefan Sōldner Rembold who helped nurse the thesis to its finish.

The result described in this thesis literally wouldn't have been possible without the other members of the crack WZ to muons team at DØ. To Emily Nurse for being a pleasure to work with, even in the wee small hours, and in general providing a model of efficiency. To Gavin Hesketh for helping to get me started in the first place and offering famus advice at the end. To Frédéric Déliot and Boris Tuchming for providing the energy that was missing from the $\mathrm{W} \rightarrow \mu \nu$ analysis. And to Dmitri, Ia, Junjie, Michiel, Marco, Sarah and all the other members of the electroweak group for their helpful advice and suggestions. I'd like to thank the members of the HEP group back in Manchester, in particular Fred Loebinger for pushing me in the right direction when the bosons were down and to Sabah for his agricultural assistance.

I'd also like to thank the people who helped me survive my time at Fermilab. From the village people, Amber, Dave, Kyle, Lydia, Raymond, Sinead, Stephen, Tim, for the nights at the losers. To the downtown folk, Matt, James and Marcus for operating the best hotel east of the Mississippi and Ben, Dustin, Marilyn, Martin, Nicola, Simon, Tamsin and everyone else, for reminding me that there are other bright lights apart from Naperville. 
Of course there were people to brighten the cloudy Manchester days as well. Thanks to Carl, Irina, Marta, Mitch and the myriad of Sarahs, I do still remember what you look like. Finally I'd like to thank my parents and the rest of my family for their constant and unconditional support. 


\section{Chapter 1}

\section{Introduction}

This thesis presents a measurement of the width of the $\mathrm{W}$ boson from the ratio of the $\mathrm{Z} \rightarrow \mu^{+} \mu^{-}$to $\mathrm{W} \rightarrow \mu \nu$ cross sections. The $\mathrm{W}$ boson is one of the fundamental particles of matter and understanding its properties, such as its width, can help in understanding the fundamental theory that dictates the behaviour of all particles. The analysis was performed using data collected at $\mathrm{D} \varnothing$, a large multipurpose detector at the most powerful running particle accelerator in the world, the Fermilab Tevatron.

The first three chapters provide a broad background to the measurement made in this thesis. Chapter 2 commences with an overview of the best theoretical description of particle physics at present, the Standard Model. It concludes by describing some of the recent experimental results and examining the direction of future investigation. Chapter 3 provides a description of the apparatus used to collect and reconstruct the data utilised by this thesis. Chapter 4 provides a description of the Monte Carlo simulations used to compare theoretical predictions, such as those described in Chapter 2, with what is seen in a real detector, such as that described in Chapter 3.

The next four chapters describe all the elements needed for the measurement of 
the $\mathrm{W}$ width. After describing the general field of electroweak physics at the Tevatron, Chapter 5 details the technique used to extract the $\mathrm{W}$ width from the ratio of the $\mathrm{W} \rightarrow \mu \nu$ and $\mathrm{Z} \rightarrow \mu^{+} \mu^{-}$cross sections. The determination of the $\mathrm{W} \rightarrow \mu \nu$ and $\mathrm{Z} / \gamma^{*} \rightarrow \mu^{+} \mu^{-}$cross sections is described in Chapter 6 . The next two chapters consist of studies performed to enable the central measurement to be made. Chapter 7, describes work performed to obtain a systematic technique to evaluate the uncertainty due to choice of PDF, one of the largest sources of systematic on the central measurement. The penultimate chapter, Chapter 8 , describes work on developing the parameterised detector simulation, PMCS, that is used to calculate the efficiency of the $\mathrm{W} \rightarrow \mu \nu$ and $\mathrm{Z} / \gamma^{*} \rightarrow \mu^{+} \mu^{-}$analyses.

The thesis culminates in Chapter 9 in a measurement of the width of the $\mathrm{W}$ boson from the ratio of the $\mathrm{W} \rightarrow \mu \nu$ to $\mathrm{Z} \rightarrow \mu^{+} \mu^{-}$cross sections. Section 9.1 describes the theoretical correction used to obtain the $\mathrm{Z} \rightarrow \mu^{+} \mu^{-}$cross section correction from the $\mathrm{Z} / \gamma^{*} \rightarrow \mu^{+} \mu^{-}$cross section. This is followed by Section 9.2 which calculates the ratio of the $\mathrm{W} \rightarrow \mu \nu$ to the $\mathrm{Z} \rightarrow \mu^{+} \mu^{-}$cross section, concentrating on particular on correlations between the two. This ratio is used to calculate the width of the W boson in Section 9.3 which is compared to the Standard Model prediction and other experimental measurements in Section 9.4. 


\section{Chapter 2}

\section{Overview of Particle Physics}

This chapter is intended to introduce some of the broader concepts in theoretical and experimental particle physics. A more detailed theoretical background to the measurement made in this thesis is provided in Chapter 5 once the description of the experimental equipment and Monte Carlo simulations have been given.

This chapter is divided into two main sections. The first section briefly outlines the theory, the Standard Model, used to describe fundamental particles and their interactions. The interested reader is referred to works such as [1] for more details. The second section gives a precis of recent and future experiments, concentrating on those searching for theories more fundamental than the Standard Model.

\subsection{The Standard Model}

The Standard Model has come to be accepted as the best description of fundamental particle physics, providing good agreement with present experimental results. It contains some arbitrary elements. For example, the exact number and masses of the fundamental particles are inserted by hand. However, as yet, no better theory 
exists. Other theories have been proposed which postulate some solutions to these problems, but no conclusive evidence to support any of these theories has been found.

This section describes all the fundamental particles of the Standard Model and the interactions between them. These interactions were thought to consist of four fundamental forces; the gravitational, the electromagnetic and the strong and the weak nuclear forces. Although the electromagnetic and weak forces appeared to be separate phenomena, they have been recognised to be different aspects of a unified, electroweak, force. The Standard Model makes no attempt to describe the gravitational interaction as it is too weak over the distances probed in present and near future experiments. The fundamental particles are listed in Section 2.1.1, the electroweak interaction is described in Section 2.1.3 and the strong interaction described in Section 2.1.4.

The success of the Standard Model lies not in cataloging these particles and interactions, but in providing a rigorous mathematical framework to describe them. Particles are considered to be excited states of quantum fields and Quantum Field Theories (QFT) are used to describe their interactions as detailed in Section 2.1.2. Each of the fundamental forces is mediated by a field and interactions between particles are associated with excited states of these fields. These excited states are themselves associated with particles, known as gauge bosons.

\subsubsection{Fundamental Particles}

In the Standard Model matter is made up of point-like fermions and bosons. The fermions can be divided into two families, namely leptons and quarks. There are three generations of fermions, each generation containing a pair of fermions from each family. These are listed in Table 2.1.

For each of the particles in Table 2.1 there exists a corresponding antiparticle. 


\begin{tabular}{|c|c|c|c|c|c|c|}
\hline \multirow{2}{*}{ G } & \multicolumn{3}{|c|}{ Leptons } & \multicolumn{3}{c|}{ Quarks } \\
\cline { 2 - 7 } & Particle & Charge & Mass $(\mathrm{MeV})$ & Particle & Charge & Mass $(\mathrm{MeV})$ \\
\hline \multirow{2}{*}{ I } & electron $(e)$ & $-e$ & 0.511 & up $(u)$ & $\frac{2}{3} e$ & $1.5-4$ \\
& $e$ neutrino $\left(\nu_{e}\right)$ & 0 & $<3 \times 10^{-6}$ & down $(d)$ & $-\frac{1}{3} e$ & $4-8$ \\
\hline \multirow{2}{*}{ II } & muon $(\mu)$ & $-e$ & 105.7 & charm $(c)$ & $\frac{2}{3} e$ & $1150-1350$ \\
& $\mu$ neutrino $\left(\nu_{\mu}\right)$ & 0 & $<0.19$ & strange $(s)$ & $-\frac{1}{3} e$ & $30-50$ \\
\hline \multirow{2}{*}{ III } & tau $(\tau)$ & $-e$ & 1777.0 & top $(t)$ & $\frac{2}{3} e$ & $1.74 \times 10^{5}$ \\
& $\tau$ neutrino $\left(\nu_{\tau}\right)$ & 0 & $<18.2$ & bottom $(b)$ & $-\frac{1}{3} e$ & $4100-4900$ \\
\hline
\end{tabular}

Table 2.1: List of fermions in the Standard Model, each with mass and electric charge. The left hand column indicates the generation $(G)$. All numbers are obtained from [2].

Some of the properties of the antiparticles, such as mass and spin, are identical to those of the particle. Other properties, such as charge and magnetic moment, are of equal magnitude but opposite sign.

There exists a number of common features between the generations. Each has an electron type lepton, with charge $-e$, an electrically neutral neutrino, and two quarks, one with electric charge of $\frac{2}{3} e$ and one with $-\frac{1}{3} e$. Here $e$ is the amount of electric charge of the electron. There are many other properties, including colour charge and parity, in which the generations show their commonality.

The principle difference between the generations arises from the differing masses of the particles. The trend is that the masses of particles increase with the generations. The question of why is one of the unsolved questions in particle physics at present. The masses of the quarks, with the exception of the top quark, are not known well, because they are only ever observed in bound states. This necessitates the use of models to extract the bare quark masses, with an associated uncertainty. The models used to extract the masses are those used by the PDG [2]. 


\begin{tabular}{|c|c|c|c|c|}
\hline Boson & Charge $(e)$ & Mass $(\mathrm{GeV})$ & Force & Couples to \\
\hline gluon $(g)$ & 0 & 0 & Strong & All quarks \\
photon $(\gamma)$ & 0 & 0 & Electroweak & $e$-type leptons and quarks \\
$W$ & 1 & $80.425 \pm 0.038$ & Electroweak & All leptons and quarks \\
$Z$ & 0 & $91.1876 \pm 0.0021$ & Electroweak & All leptons and quarks \\
\hline
\end{tabular}

Table 2.2: List of fundamental gauge mediating bosons in the Standard Model. All numbers are obtained from Reference [2].

Table 2.2 lists the bosons associated with the fundamental forces. The gluon mediates the strong force and only couples to quarks and gluons. The unified electroweak force is carried by the photon, which couples to all electrically charged particles, and the $\mathrm{W}$ and $\mathrm{Z}$ bosons, which couple to all fermions and each other.

\subsubsection{Quantum Field Theories}

The Standard Model describes particles in terms of excited states of quantum fields. The coupling of different particles to each other arise naturally when symmetry requirements are imposed on the fields.

These symmetry requirements dictate that the field must be unchanged under some gauge transformation. The exact nature of the symmetry is defined by the particular group of transformations. However there is no reason why this transformation has to be the same over all space time coordinates, hence this requirement is known as local gauge invariance. In the same way that requiring that a system is symmetrical under rotations produces the conservation of angular momentum, the imposition of gauge invariance also introduces quantities, denoted charge, conserved by the field.

The transformations considered here are based upon a series of unitary, spacetime dependent, matrices acting on the fermion and boson fields. The simplest 
set of matrices are denoted $\mathrm{U}(1)$ and consist of a group of unitary one-dimensional matrices. In general, the matrices required are more complex than those of the $\mathrm{U}(1)$ group, and $\mathrm{SU}(\mathrm{n})$ groups are used. Special Unitary, SU(n), groups have the additional requirement that the determinant of the matrix must be unity. They consist of a set of $n \times n$ matrices, with $n^{2}-1$ generators, where the generators are a set of orthogonal matrices.

In general groups with $n$ greater than one are non-commutative, which means that different results are obtained depending on the order that transformations are performed. This has the result that the gauge fields can interact with themselves, producing coupling between the gauge bosons.

The Standard Model is represented by a combination of three different groups, $\mathrm{SU}(3) \otimes \mathrm{SU}(2) \otimes \mathrm{U}(1)$. The $\mathrm{SU}(3)$ group corresponds to the strong interaction, discussed in more detail in Section 2.1.4, and the $\mathrm{SU}(2) \otimes \mathrm{U}(1)$, corresponds to the unified electromagnetic and weak interactions, or electroweak interaction, discussed in more detail in Section 2.1.3. Again this section provides only a cursory overview of the theory. An interested reader is directed to [3] for more details.

\subsubsection{The Electroweak Interaction}

The first quantum field theory to be devised was the U(1) theory used to describe the interactions between an electron and a photon. This theory, known as Quantum ElectroDynamics (QED), has since been extended to describe all fundamental electromagnetic interactions. The imposition of the condition that the theory is invariant under these $\mathrm{U}(1)$ transformations introduces the photon field and a conserved physical quantity, electric charge. QED provides a mathematical description of the interactions between particles possessing this charge and the photon field. 


\section{Feynman Diagrams and Perturbation Theory}

To give an illustration of how quantum field theory is used, consider the process $\mathrm{e}^{+} \mathrm{e}^{-} \rightarrow \mu^{+} \mu^{-}$, which can be modelled using QED alone at low energies. The simplest way that this process can proceed is shown in the left hand diagram of Figure 2.1. These diagrams are known as Feynman diagrams and are widely used to illustrate fundamental processes. The horizontal axis in these diagrams represents time and the vertical axis represents spatial separation. Figure 2.1(a) shows an electron and a positron approaching each other, annihilating to produce a virtual photon $\left(\gamma^{*}\right)$ which itself decays to a muon antimuon pair. All fundamental processes can be illustrated using Feynman diagrams such as these.

Virtual particles exist only for short periods of time, short enough that although they have well defined quantum numbers, such as electric charge, they violate energy and momentum conservation. For instance in Figure 2.1 the invariant mass, in the centre of mass frame, of the colliding system is non-zero. This implies that the exchanged photon has a non zero mass, whereas a real photon is massless. This arises from the Heisenberg Uncertainty Principle:

$$
\Delta E \Delta t>1
$$

where $\Delta E$ is the uncertainty of the energy of the state and $\Delta t$ is related to the lifetime of the state [4]. Note that throughout this thesis natural units are used, where Planck's constant, $\hbar$, and the speed of light, $c$, are set to unity.

Feynman diagrams are more than just a means of providing clarity in the way that an interaction proceeds; they are an invaluable aid in calculating observables such as cross sections. Each particle has a field associated with it and each coupling between the particles has some associated factor. The Feynman diagram can be used as an accounting tool to tally all required fields and couplings to calculate 


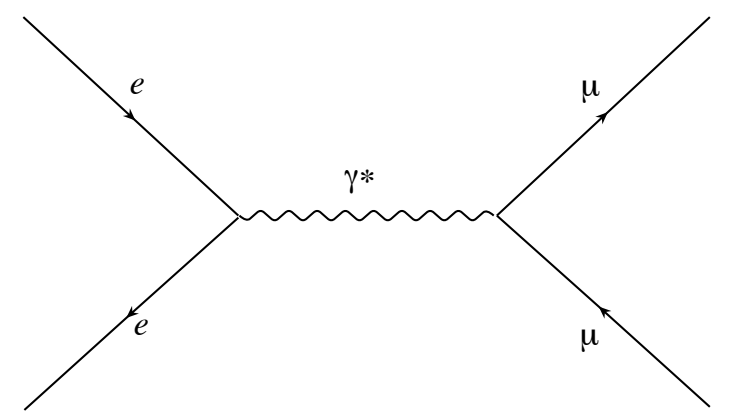

(a)

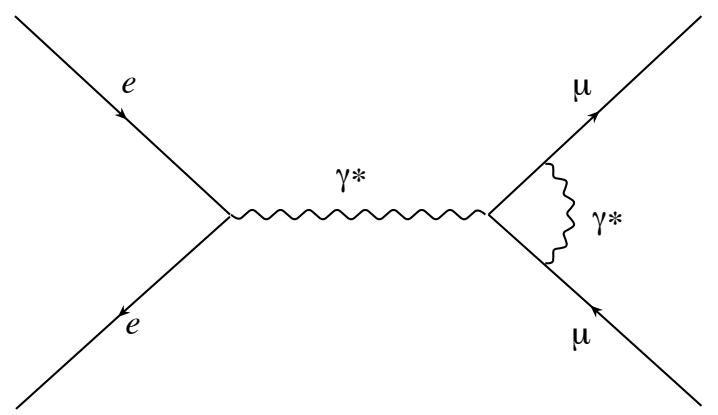

(b)

Figure 2.1: Feynman diagrams showing the process $\mathrm{e}^{+} \mathrm{e}^{-} \rightarrow \mu^{+} \mu^{-}$. The left hand diagram shows this at tree level, the right hand diagram shows this with one of the first order corrections.

the amplitude for a given process. From the amplitude the cross section can be determined.

In addition, there are radiative corrections to every process in QFT. Radiative corrections arise due to the presence of Feynman diagrams with additional field lines. Consider Figure 2.1(b) which only differs from Figure 2.1(a) in that there is an internal virtual photon loop added. Alternatively one of the final state muons might radiate a real photon with insufficient energy to be detected. To an observer who can only detect the final states, these two processes are indistinguishable from that shown in Figure 2.1(a). Similarly more and more loops can be added, to provide more and more complicated ways of obtaining the final state. A truly comprehensive calculation would have to include an infinite number of these diagrams.

There is a technique that is used to surmount this problem and that is to treat the calculation as a perturbation series expanded in powers of the coupling constant, $\alpha_{E W}$ in this case. This is possible because every vertex introduces a new factor of $\alpha_{E W}$. Therefore the first, leading order (LO), term in the expansion includes only the tree level diagram, the next to leading order (NLO) term includes only one 
loop corrections and so on. The term tree level is applied to the diagram with no radiative corrections.

As $\alpha_{E W}$ is much smaller than one, higher order terms can be neglected. The point at which higher order terms can be neglected depends on the accuracy desired. The total amplitude is determined by summing the amplitudes of all diagrams, up to the level of accuracy required. This sum is squared and integrated over available phase space to produce the cross section.

\section{The Unified Electroweak Interaction}

The weak interaction, responsible for phenomena such as $\beta$ decay, was less easy to model. Glashow, Salam and Weinberg were able to provide a model by uniting the electromagnetic and weak interactions into a single, unified theory, which has been denoted electroweak theory.

It consists of two groups, $\mathrm{SU}(2) \times \mathrm{U}(1)$, which are associated with the conservation of weak isospin and hypercharge respectively. There are three generators associated with the $\mathrm{SU}(2)$ group that correspond to the $W^{+}, W^{-}$and the $W^{0}$ fields. The generator associated with the $\mathrm{U}(1)$ group corresponds to the $B^{0}$ field.

The bosons associated with the $\mathrm{W}^{ \pm}$fields have been experimentally observed, but no boson directly associated with the $\mathrm{B}^{0}$ or $\mathrm{W}^{0}$ fields are seen. This is because the $\mathrm{W}^{0}$ and $\mathrm{B}^{0}$ fields interfere with each other. The bosons experimentally observed are associated with two orthogonal combination of these states, the $Z$ boson, which couples only to weak charge, and the photon, which couples only to electric charge.

The fact that all the transmitters of the weak interaction are massive limits the range of the interaction at energies much less than the masses of these bosons, making the interaction appear weak. At high energies the strength of the electric and weak interaction are comparable. 


\section{The Higgs Mechanism}

Despite the successes of the electroweak model, it has one significant problem; the gauge bosons are predicted to be massless. A mechanism is required to provide mass to the gauge bosons without destroying the gauge invariance of the theory. This process is known as electroweak symmetry breaking.

The Standard Model does this by introducing a new field, the Higgs field. This field has a potential which is non zero in the vacuum. It is said to have a non zero vev (vacuum expectation value). The interaction with this field leads to the acquisition of mass by the particles of the Standard Model, such as the W and Z bosons [5].

\subsubsection{The Strong Interaction}

As the electromagnetic force is modelled by QED, so the strong force is modelled by Quantum ChromoDynamics (QCD). QCD is an SU(3) theory based upon the conserved quantity, colour. All quarks have one colour, either red, green or blue. All leptons are colourless and so do not interact via the strong force.

The imposition of the condition that the theory is invariant under the colour $\mathrm{SU}(3)$ transformations, as well as introducing the colour charge, gives rise to the gluon field. As there are eight generators there are eight types of gluon field, each with different combinations of colour charge. The fact that gluons have colour charge means that they couple to themselves.

The main feature of the strong force is the dependence of the strong coupling constant, $\alpha_{\text {strong }}$, on energy. Whereas the electromagnetic coupling effectively become weaker as the energy of an interaction decreases, $\alpha_{\text {strong }}$ effectively increases as energy decreases, rising above unity at low energies. This makes the perturb-

ation techniques employed above impossible to use for low energy QCD. Another consequence is that the strong force increases with distance, resulting in the absence 
of any coloured states.

Quarks are only observed in colour neutral bound states, known as hadrons. In an analogous way that electrically neutral states may be obtained by adding an equal amount of positive and negative charge, colour neutral states may be obtained by adding an equal amount of red, green and blue charge. There are two types of hadrons that have been observed at the present; baryons consisting of three quarks, one of each colour and mesons consisting of a quark and an antiquark where the colour of the quark mirrors that of the antiquark. Other more complex bound states have been proposed, but have not been confirmed to exist.

The compound nature of hadrons, such as protons, complicates the calculation of observables, such as cross sections. All calculations are performed in terms of the fundamental constitiuents of the hadrons, known as partons. The obtention of any observable involves summing over the different partons in the hadron.

\subsection{Current Directions in Particle Physics}

This section describes the current experiments in particle physics and the phenomena they are probing. The first section will give a brief description of experiments from the recent past, the present and the near future. Unless otherwise stated all results are obtained from [2].

\subsubsection{Recent and Future Experiments}

The reason why high energy physics is interesting is summed up by this equation:

$$
\Delta E \Delta t>1
$$


This equation implies that the higher the energy scale $(E)$ used the smaller the timescales $(t)$ and distances probed. Therefore to look at more fundamental scales higher energies have to be obtained. In the main these high energies have been obtained using particle accelerators. The next chapter, Chapter 3, gives a more detailed explanation of the workings of one particular accelerator, the Tevatron.

Over the course of the last century, as accelerators have been able to produce particles with higher and higher energies, smaller distance scales have been resolved revealing that the atom, once considered to be indivisible, consists of electrons, protons and neutrons and subsequently that these protons and neutrons consist of quarks and gluons.

This section does not seek to provide an exhaustive history of particle physics experiments, but merely concentrates on the experiments that have produced results that are mentioned in this thesis. The first experiments are the UA1 and UA2 experiments in the 1980s where electroweak bosons were first observed directly [6]. The UA experiments were on the CERN SppsS that collided beams of protons and antiprotons together to produce a $\mathrm{p} \overline{\mathrm{p}}$ centre of mass energy of up to $900 \mathrm{GeV}$. They detected and reconstructed enough events attributed to $\mathrm{W}$ and $\mathrm{Z}$ bosons to be able to prove their existence and to make measurements of some of their properties, such as mass.

The problem with $\mathrm{p} \overline{\mathrm{p}}$ accelerators is that as it is the constituent partons colliding it is impossible to control the energy of the interaction. This problem can be solved by colliding electrons and positrons where all the accelerated energy is involved in the collision ${ }^{1}$.

There were two large $\mathrm{e}^{+} \mathrm{e}^{-}$colliders in the 1990s, the CERN LEP collider [7] and the Stanford Linear Accelerator [8]. Both operated at a centre of mass energy at the

\footnotetext{
${ }^{1}$ The emission of radiation from the colliding particles means that this statement is not quite true. However the effect is not large when compared to the spread of centre of mass energies at a $\mathrm{p} \overline{\mathrm{p}}$ collider.
} 
mass of the $\mathrm{Z}$ boson, acting as $\mathrm{Z}$ factories. This allowed huge samples of $\mathrm{Z}$ bosons to be produced, resulting in a precise understanding of the properties of the $\mathrm{Z}$ boson [9].

The LEP accelerator also operated at higher centre of mass energies allowing the study of W pair production [10] and a search for the Higgs boson to be made [11]. Since $\mathrm{W}$ bosons had to be produced in pairs and not singly, on resonance, the sample of $\mathrm{W}$ bosons was much smaller than the sample of $\mathrm{Z}$ bosons. Even so large enough samples were produced that allowed many useful measurements to be made. There was no conclusive evidence for new physics from these experiments, either in direct searches or in tests of the Standard Model. The large amount of data and the cleanness of this data resulted in a large number of precision tests of the Standard Model.

Although the $\mathrm{e}^{+} \mathrm{e}^{-}$colliders had many successes, the centre of mass energy available was restricted by radiation from the accelerated beams. To probe higher energies hadron colliders are now being run and constructed. The p $\bar{p}$ collider running at the moment is the Tevatron, which has been running since the 1980s $[12,13]$. The first running period (Run I), with a centre of mass energy of $1.8 \mathrm{TeV}$, lasting until 1995, during which the top quark was first observed $[14,15]$ and enough W bosons were produced to allow a measurement of the mass of the $\mathrm{W}$ boson which was a great improvement on those made at UA1 and UA2 [16].

Since then the accelerator and experiments have been upgraded for a new run (Run II) with a higher centre of mass energy (1.96 TeV) and greater rate and intensity of collisions. The intensity that the beams are collided is related to a quantity known as the luminosity. As this is the experimental environment from which the result described in this thesis is obtained, the accelerator and one of the experiments is described in more detail in Chapter 3.

The next accelerator due to start working will be the CERN LHC [17] which will collide two beams of protons at a centre of mass energy of $14 \mathrm{TeV}$ and higher lumin- 
osities than the Tevatron. These high energies and high luminosities should be able to probe areas of new physics unreachable at previous generations of accelerators, in particular it should allow greater understanding of electroweak symmetry breaking to be obtained.

These are just a few of the experiments concentrating on the high energy frontier. There are many other experiments that instead of probing the highest energies measure a certain quantity or set of quantities to high precision. The range of measurements and energy scales complement each other, testing different facets of the Standard Model.

\subsubsection{Current Avenues of Research}

The Standard Model has been extremely successful, providing excellent agreement between theoretical calculations and data. This agreement varies from very high energies, such as the mass of the top quark as measured directly and predicted using other parameters of the Standard Model, to very low energies, such as the corrections to the magnetic moment of the electron.

Notwithstanding the many successes of the Standard Model there are still concerns associated with it [3]. The first concern is principally aesthetic, with there being a number of parameters determined empirically and, seemingly arbitrarily. For example there are three generations not two or four. It is argued that a theory which cannot explain why the parameters take certain different values cannot be truly fundamental, that the order of the generational structure indicates hidden structure in an analogous way to the periodic table of the elements points to their atomic structure. These arguments may be true, but are insufficient in themselves to undermine the Standard Model. There are however several more indications that the Standard Model is wanting.

Not all the particles of the Standard Model have been observed, with no experi- 
mental evidence for the Higgs boson, the excited state of the Higgs field. Direct searches at present preclude a Higgs boson with a mass less than $114.1 \mathrm{GeV}$ (at 95\% confidence level), which still leaves possible a Standard Model Higgs boson. The increased energy and luminosity of the LHC should either detect the presence or exclude the existence of the Standard Model Higgs boson [18].

Even if the Higgs boson is discovered, there are still questions about the Standard Model. One of these is the hierarchy problem, which relates to the mass of the Higgs boson, $M_{H}$. This is constrained by other parameters of the Standard Model to be around the electroweak scale, around $100 \mathrm{GeV}$. Virtual corrections to $M_{H}$ arising from loops of Standard Model particles, should introduce corrections to $M_{H}$ up to the scale at which the Standard Model remains valid. The fact that this is not the case has various possible explanations.

One is that this is just a case of fine-tuning, that the corrections just happen to cancel out, which is felt by many to be aesthetically displeasing. Another is that this is an indication of new, Beyond the Standard Model (BSM), physics. The perennially favoured theory to explain the hierarchy problem is known as SuperSymmetry (SUSY). SUSY postulates that every fermion has a bosonic partner and every boson a fermionic one. The hierarchy problem is trivially solved as the virtual corrections contributed by fermions and bosons now exactly cancel. The fact that these partners are not seen as yet indicates that if SUSY is a true symmetry it must be broken in some way, yet to be understood.

Another feature that favours SUSY is the behaviour of the running of the effective coupling constants. The coupling of two particles depends on the energy of the interaction, with the strong and weak coupling constants becoming weaker as the energy increases and the electromagnetic coupling constant becoming stronger. If SUSY is true these coupling constants run in such a way that they all meet at a single point, which advocates of SUSY claim is an indication that SUSY provides a fundamental theory [19]. There are other aspects of the Standard Model that are 
wanting, these are listed below.

The Standard Model was formulated with the assumption that neutrinos are massless. The results from neutrino oscillation experiments prove that this is not the case [20]. The implications of this result have yet to be fully understood and incorporated into the model.

The Standard Model does not successfully incorporate gravity. There is a proposed theory of quantum gravity, but this has yet to be formulated and the proposed gauge boson associated with this theory has yet to be observed. As gravity is not significant at the distances probed by the present generation of experiments this does not affect the interpretation of these results. However gravity remains one of the fundamental forces, and as such a complete model should include it.

The Standard Model only attempts to model a small proportion of the contents of the Universe. Astronomical observations indicate that most of the Universe is made up of Dark Matter and Dark Energy, neither of which is included in the Standard Model. Dark Matter is matter that cannot be directly observed by us and is required to explain the rotation of distant galaxies. This mass distribution indicates that the matter must be sub-relativistic, excluding light particles such as neutrinos. The best candidate for this dark matter are some yet to be discovered BSM particle, possibly from SUSY. Dark Energy is a new phenomenon, invoked to explain the increasing rate of expansion of the Universe, and has yet to be fully incorporated into any model of particle physics, but which will need incorporating into any complete theory.

Therefore new models are proposed to reconcile some or all of these concerns with the many successes of the Standard Model. These theories make phenomenological predictions about what could be observed in experiments. These signals are searched for as described in the next section. 


\section{Looking for Physics Beyond the Standard Model}

There are two forms in which BSM physics can be observed, both of which are looked for. The first is as an excess, in a particular experimental signal, over what is predicted by the Standard Model. One such example, as described in Section 8.3.2, is the search for heavy $\mathrm{Z}$ bosons, which would show as a excess over the Standard Model prediction in the di-muon mass spectrum.

The second is by overconstraining the model, making a greater number of precise measurements than Standard Model parameters. One example can be taken from Figure 2.2 [10]. It shows the relationship between the masses of the $\mathrm{W}$ boson, $M_{W}$, the top quark, $M_{t}$, and the Standard Model Higgs boson, $M_{H}$. It is possible to infer $M_{H}$ if other Standard Model parameters, including $M_{W}$ and $M_{t}$, are known. This is done through the contribution the Higgs boson makes to the radiative corrections in the measurement of $m_{t}$ and $M_{W}$. Therefore if and when the Higgs boson is discovered the a direct measurement of $M_{H}$ will overconstrain the parameters of the Standard Model, testing its validity.

Another example of an indirect measurement is enclosed in this figure, in that $M_{W}$ and $M_{t}$ are determined not only from direct measurements, but also indirectly. Indirect measurements of quantities are produced from other experimental results by using relationships from the Standard Model. The measurement made in this thesis is an example of an indirect measurement, producing the value of the $\mathrm{W}$ width from the ratio of the $\mathrm{W} \rightarrow \mu \nu$ to $\mathrm{Z} \rightarrow \mu^{+} \mu^{-}$cross sections. The requirement that the direct and indirect measurements of $M_{W}$ and $M_{t}$ must agree also acts as a test of the Standard Model. 


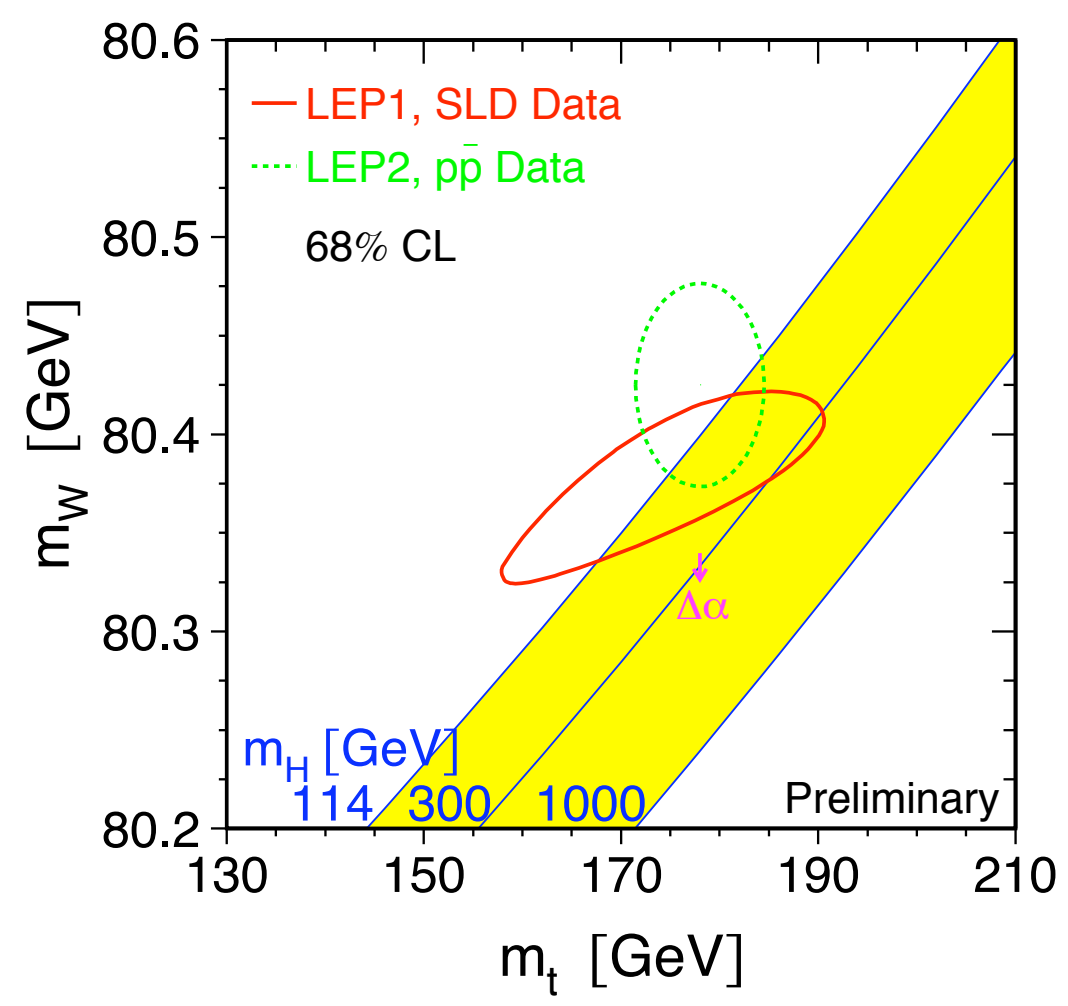

Figure 2.2: The mass of the $\mathrm{W}$ boson, $M_{W}$, plotted against the mass of the top quark, $M_{t}$. The yellow band shows the allowed range of values for the Standard Model Higgs boson. The green ovoid shows the constraints placed upon $M_{W}$ and $M_{t}$ by direct measurement. The red ellipse shows the constraints placed upon these values by indirect measurements [10]. 


\section{Chapter 3}

\section{Experimental Apparatus}

The high energies required to probe the fundamental constituents and interactions of matter are obtained using particle accelerators, such as the Fermilab Tevatron. These machines obtain these high energies by accelerating a beam of charged particles, using electromagnetic fields. This energy is released, allowing new particles to be produced, by colliding this beam with either a fixed target or with another beam.

The Tevatron is a large, circular accelerator used to accelerate a beam of protons and a beam of antiprotons, each to $0.98 \mathrm{TeV}$. There are two places on the ring where the beams are focused together to produce collisions. At one of these points lies the $\mathrm{D} \emptyset$ detector, whilst at another one is the CDF detector. Both of these are large multipurpose detectors, with many sub-systems to allow the study of many kinds of particles.

The remainder of this chapter gives a brief outline of the machinery used to accelerate and collide the particles and to study the products of these collisions. This starts with a summary of the Tevatron in Section 3.1 and concludes with a description of the $\mathrm{D} \varnothing$ detector in Section 3.2. 


\subsection{The Fermilab Tevatron}

The Tevatron collides a beam of protons and a beam of antiprotons with a centre of mass energy of $1.96 \mathrm{GeV}$, making it the highest energy facility in the world until the LHC commences operation.

The acceleration of the particles collided in the Tevatron involves several stages of which it is the final stage. Figure 3.1 shows a schematic view of the Fermilab accelerator chain. A detailed description of the construction and operation of the accelerator chain can be found in [12] and [13].

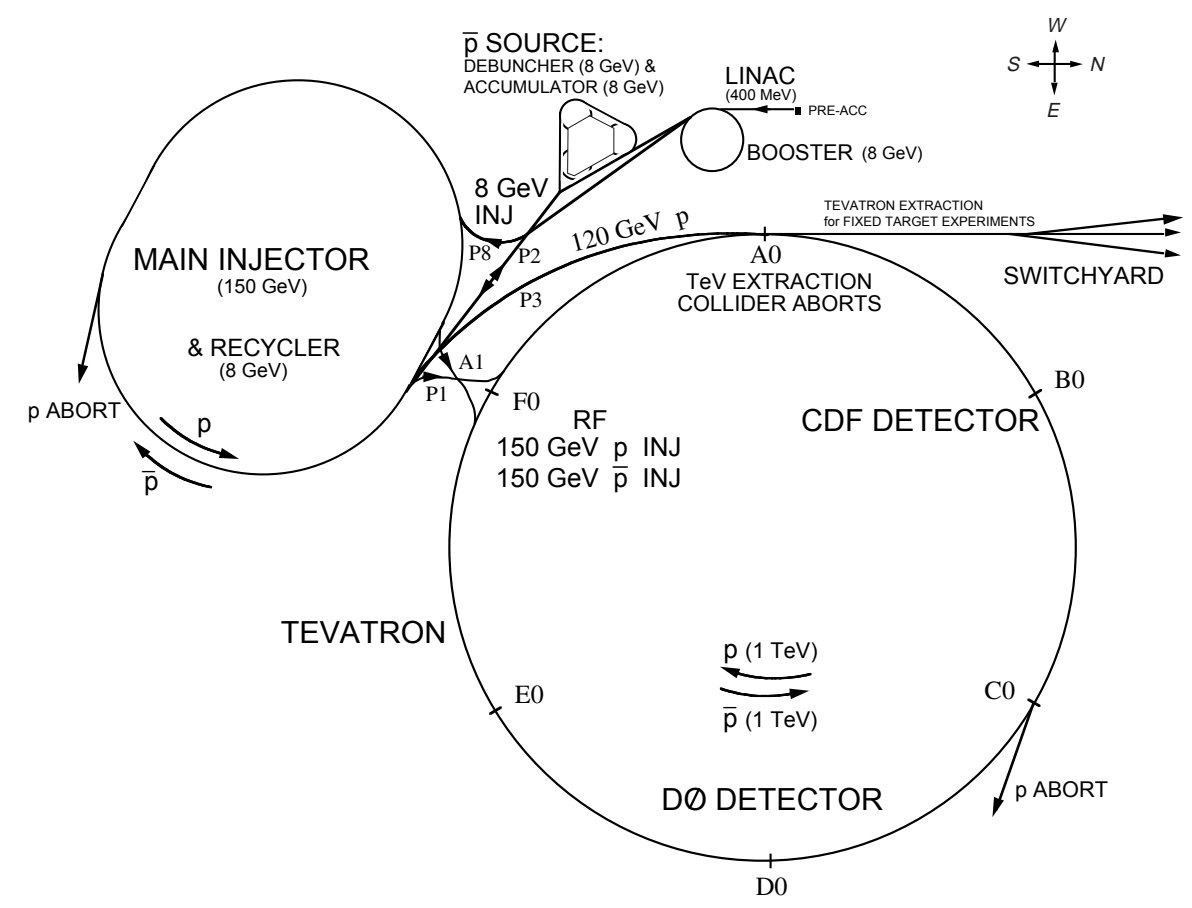

Figure 3.1: Drawing of the Fermilab accelerator chain.

The acceleration of the protons begins with a beam of $\mathrm{H}^{-}$ions inside a CockcroftWalton accelerator, which accelerates them to $750 \mathrm{KeV}$. The ions are passed to a linear accelerator, the Linac, which accelerates them to $400 \mathrm{MeV}$. The electrons are stripped off by passing the $H^{-}$beam through a graphite foil, leaving a beam 
of protons. The protons are accelerated to $8 \mathrm{GeV}$ by the booster synchrotron ring and from there are transferred to the Main Injector, a larger synchrotron ring, which accelerates them to $150 \mathrm{GeV}$. In the Main Injector the protons are grouped into bunches before being transferred to the Tevatron which accelerates them to $0.98 \mathrm{TeV}$.

The antiprotons are produced by directing $120 \mathrm{GeV}$ protons from the Main Injector into a copper-nickel target. One of the products of this procedure are antiprotons. The antiprotons are collected and transferred to the debuncher where the beam is cooled and accelerated to $8 \mathrm{GeV}$ before being passed to the accumulator, a storage ring. From the accumulator they are transferred to the recycler, an accelerator in the same tunnel as the Main Injector. When a sufficient quantity of antiprotons has been produced, they are gathered into bunches and passed into the Main Injector. In the Main Injector the antiprotons are accelerated up to $150 \mathrm{GeV}$, before being passed into the Tevatron where they circulate in a direction counter to that of the protons.

The protons and antiprotons do not pass round the Tevatron in a continuous stream. Both the protons and antiprotons are gathered together into 36 bunches, which are themselves gathered into 3 superbunches, each containing 12 bunches. The superbunches are separated by $2 \mu$ s and the bunches within the superbunch by 396 ns.

Although the beams travel round the same accelerator they are kept apart most of the time, only being focused together in two places. Around each of these two points are built large multipurpose detectors, CDF and D $\varnothing$, which are used to study the product of interactions between the beams. The collisions are not confined to a single point, but occur with an approximately Gaussian distribution. The width of this Gaussian is small transverse to the beams (about 30 microns), but in the direction parallel to the beams it is quite extensive, having a width of about $25 \mathrm{~cm}$. The region in which the collisions occur is known as the beamspot. 


\subsection{The DØ Detector}

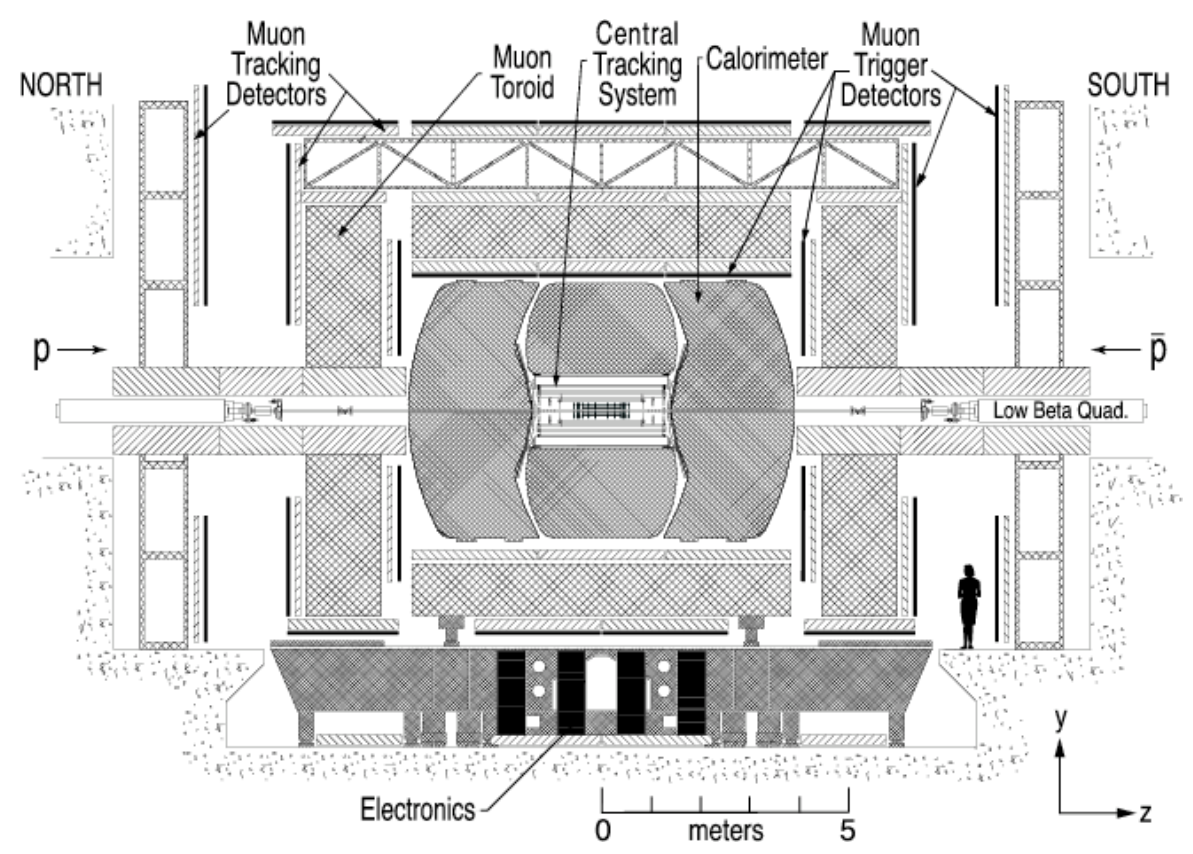

Figure 3.2: Cross section of the $\mathrm{D} \varnothing$ detector

The $\mathrm{D} \varnothing$ detector, shown in cross section in Figure 3.2, was designed to allow the study of the wide range of particles produced in high energy p $\bar{p}$ collisions. A detailed description of the construction of the detector can be found in [21], from which all figures and numbers are taken unless otherwise stated. Many of the particles of interest decay very quickly and have to be reconstructed from their decay products. The particles that live long enough to be observed can be grouped into six distinct categories: photons, electrons, muons, neutrinos and charged and neutral hadrons. As these different particles interact in different ways the detector contains several complementary sub-systems, arranged in a series of layers from the beampipe. The major sub-systems are:

- A central tracking system, as described in Section 3.2.2.

- Electromagnetic and hadronic calorimeters, as described in Section 3.2.3. 
- The muon system, as described in Section 3.2.4.

- Luminosity monitors, as described in Section 3.2.5.

\subsubsection{The D $\varnothing$ Co-ordinate System}

It is convenient to define the co-ordinate system employed at $\mathrm{D} \emptyset$. This is a righthanded cylindrical system, with the proton beam defining the positive $z$ direction. The origin of the co-ordinate system is at the centre of the $\mathrm{D} \emptyset$ detector, which roughly corresponds to the centre of the interaction region.

Symmetries in both the detector design and in the physics processes studied mean that $\phi=\tan ^{-1}\left(\frac{y}{x}\right)$ and $\theta=\tan ^{-1}\left(\frac{r}{z}\right)$, are often more natural coordinates to use. Because it is the partons within the proton that interact, and these have a range of momenta, the centre of mass system is in general boosted in the $z$ direction. Therefore $\theta$ is often replaced by a co-ordinate that is not so sensitive to boosts in the $z$ direction. The co-ordinate used is rapidity, $y=\frac{1}{2} \ln \left(\frac{E+p_{z}}{E-p_{z}}\right)$, or pseudo-rapidity, $\eta=-\ln \left(\tan \left(\frac{\theta}{2}\right)\right)$, which in the relativistic limit are equal. Although the absolute value of the co-ordinate changes under these boosts, its relative value with respect to another co-ordinate remains unchanged. This results in the angular sizes of physics objects, such as jets of hadrons, being uniform in terms of rapidity, allowing algorithms, such as those used to find jets, written in terms of rapidity, to be used throughout the detector. The rapidity distribution of the $\mathrm{Z}$ can also be used to provide information on the parton structure of the proton [22].

The $\eta$ co-ordinate is defined in two ways: "detector $\eta$ " relates to the position of the particle at a given layer of the detector and is measured with respect to $(0,0,0)$; "physics $\eta$ " relates to the direction of travel and is measured with respect to where the particle originated from. In the same way $\theta$ can be defined in terms of direction of travel or position within the detector. 
The fact that, in general, the momentum components of the colliding partons parallel to the beampipe is unknown makes it impossible to use the conservation of momentum to provide more information on an event. However the momentum component of the beams transverse to the beampipe, $p_{t}$, is approximately zero and therefore conservation of transverse momentum can be used. One use is to infer the presence of undetected particles, such as neutrinos, by large missing transverse energy, $E_{T}{ }^{1}$, defined as the imbalance in the transverse energy where a high $p_{t}$ particle escapes the detector undetected.

\subsubsection{The Central Tracking System}

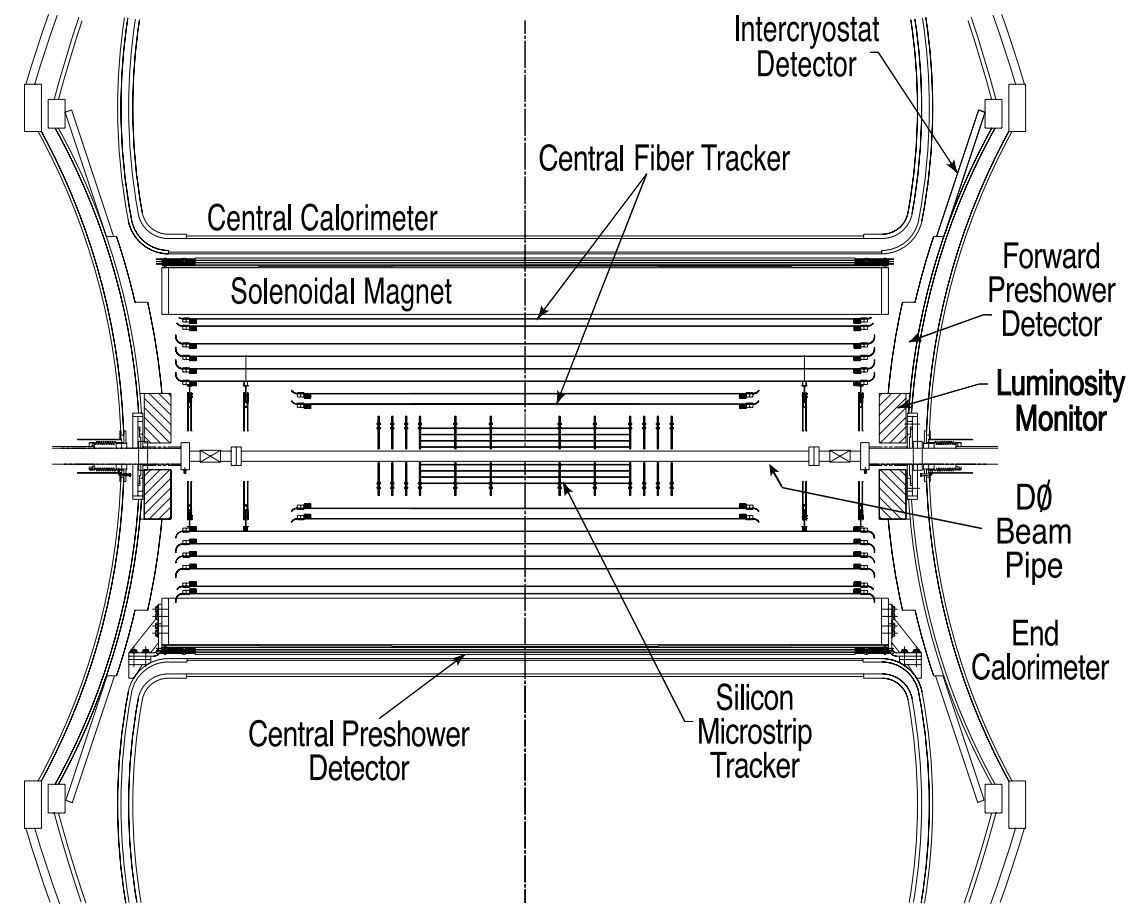

Figure 3.3: The DØ detector tracking system

The central tracking system, shown in Figure 3.3, is designed to be able to

\footnotetext{
${ }^{1}$ For ultra relativistic particles the transverse energy and transverse momentum can be treated as being the same.
} 
reconstruct the trajectories of charged particles from where they are produced within the beampipe (the vertex) to the edge of the tracking volume. Close to the beampipe is the Silicon Microstrip Tracker (SMT) which is used to accurately determine where the track originates from. This allows particles with relatively long lifetimes such as $\tau$ leptons and hadrons containing $b$ and $c$ quarks to be identified as their decay products will originate away from the beamspot at a secondary vertex. Beyond the SMT is the Central Fibre Tracker (CFT) which allows a measurement of the tracks over a more extended radius.

The whole tracking system is enclosed in a $2 \mathrm{~T}$ solenoid magnet, aligned along the beamline, which bends the path of all charged particles in the $r-\phi$ plane. The radius of curvature allows the $p_{t}$ of a particle to be measured and the direction of curvature allows the charge of a particle, $q$, to be determined.

\section{The SMT}

The active material, that is the material used to detect particles, in the SMT is a series of wafers of silicon. When a charged energetic particle passes through silicon it ionises it, creating electron hole pairs. Charged particles with energies of order $\mathrm{KeV}$ ionise the material directly. Particles with much higher energies, such as those considered in this thesis, not only ionise the silicon, but transmit many $\mathrm{KeV}$ of energy to the ionised electrons. These electrons then subsequently ionise other silicon atoms, in effect amplifying the signal. Each silicon wafer has a voltage applied across it so that the electrons will drift to one end where they can be collected. This electronic signal can then be amplified and read out.

The silicon has a relatively high density allowing the wafers that make up the SMT to be small. This allows a fine degree of granularity, giving an accurate measurement of the position of the track. The higher density of a material the greater the number of interactions between the detector and the charged particle. Each interaction 
scatters the particle through some small angle. This multiple scattering reduces the resolution of the tracking system. Therefore the SMT only consists of enough layers to constrain the starting position of the track, and the greater part of the path of a charged particle is measured using the less dense CFT.

The SMT, shown in Figure 3.4, is designed to provide tracking and vertexing over nearly the entire angular range of the calorimeters and muon system. The design is influenced by the large spread of the beamspot in $z$ and the fact that the best resolution is obtained when the path of a charged particle is perpendicular to the active material in the detector. This is because when a charged particle strikes the wafer at an angle the charge is deposited over a wider area of the wafer, blurring the measurement of position.

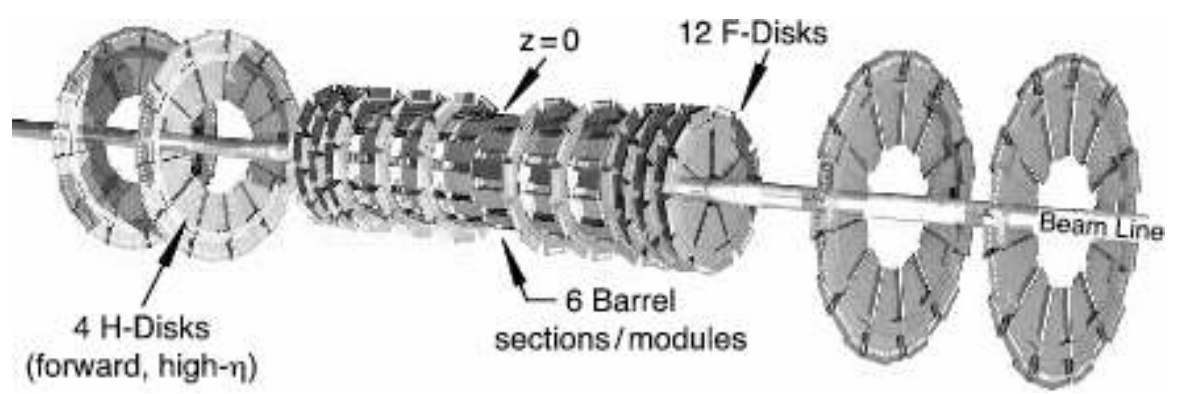

Figure 3.4: The SMT

These factors motivated the design which consists of six disc-barrel modules, with the barrels giving better resolution for tracks perpendicular to the beampipe and the disks giving better resolution for tracks parallel to the beampipe, allowing good resolution up to high $\eta$. In addition there are a further three discs at each end of the central section of the SMT and two further, more coarsely segmented disks lying beyond these. These more coarsely segmented outer discs are denoted H-Disks to distinguish them from the standard F-Disks.

Each barrel consists of 4-four layers which are constructed out of a series of silicon 
modules. The two layers closest to the beampipe are made up of 12 modules and the outer two layers consist of 24 modules. Each of the F-Disks consist of 12 wedge shaped modules and each of the H-Disks consist of 48 wedge shaped modules. These modules are constructed out of ' $\mathrm{n}$ ' type silicon with surface strips of ' $\mathrm{p}^{+}$' silicon. In addition, in some double sided modules, the opposite surface is implanted with strips of ' $\mathrm{n}^{+}$' silicon. These strips are angled with respect to each other to produce a three dimensional, stereo, position measurement.

In the barrel all the modules have strips aligned with the beam axis to allow a measurement of the $r-\phi$ position. In addition all the second and fourth layers are double sided with the strips of $\mathrm{n}^{+}$silicon at an angle of two degrees to the beam axis. In the four central barrels the first and third layers are also double sided with $\mathrm{n}^{+}$strips perpendicular to the beam axis. This second layer allows a measurement in the $r-z$ plane to be made as well.

All the wedge shaped modules in the F-Disks are double sided with the $\mathrm{p}^{+}$strips at angle of 15 degrees to the radial axis and the $\mathrm{n}^{+}$strips at an angle of -15 degrees to the radial axis, producing a stereo angle of thirty degrees. The H-Disks have a layer of 24 single sided modules mounted on each face. The strips are aligned to be at angle of 7.5 degrees to the radial on one face and -7.5 degrees on the other, producing a stereo angle of 15 degrees.

\section{The CFT}

The active material in the CFT consists of a series of scintillating fibres. When a charged energetic particle passes through a fibre the material is excited. This occurs in two stages, in an analogous way to the deposition of energy in the SMT. Firstly the energetic particle ionises the material of the CFT, then the ionised electrons excite

other molecules of the fibres. This excited state rapidly decays by the emission of a photon. 
The fibres transmit these photons to their ends where they are transfered via clear fibres to Visible Light Photon Counters (VLPCs). These are solid state devices that convert the photons to electronic signals via electron-hole pair creation. These signals are amplified and read out. The fibres of the CFT are mounted on eight concentric carbon cylinders. Each cylinder has two layers of fibres mounted on it. Each layer consists of a radial doublet of fibres, as shown in Figure 3.5. On each cylinder one of the doublet layers has the fibres aligned along the beam axis and another at an angle of three degrees to the beam axis to allow a stereo measurement.

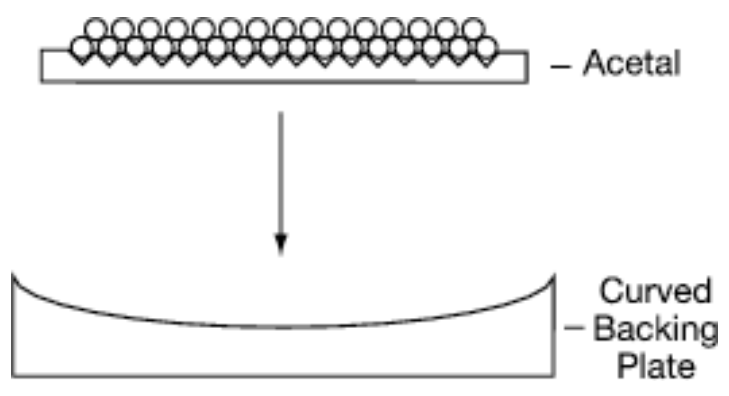

Figure 3.5: Diagram illustrating the mounting of the fibres on the carbon cylinders within the CFT. The radial doublet structure can be clearly seen.

The outer six cylinders are 2.52 meters long, but the inner two are curtailed to only 1.66 metres long to accommodate the H-discs of the SMT, as can be seen in Figure 3.3. The CFT allows a measurement of tracks up to $\eta$ of approximately 1.7.

\subsubsection{The Calorimeter}

The calorimeter is designed to measure the energy and position of most particles produced in $\mathrm{p} \overline{\mathrm{p}}$ collisions. To achieve this objective incoming particles are induced to deposit energy via the emission of other particles, a phenomenon known as showering. The properties of showers induced by particles that interact only via the electro- 
magnetic interaction, electrons and photons, are different from those that interact principally via nuclear forces, jets.

Jets arise because quarks and gluons produced in fundamental interactions cannot exist in colour singlet states. These quarks and gluons, along with some of the remnants of the proton and antiproton combine together to produce a series of hadrons travelling in the same direction as the original quark or gluon. The width and depth of the hadronic showers produced by jets can be used to distinguish them from electromagnetic showers of electrons and photons.

\section{Principles of Calorimetry}

High energy electrons, of a few hundred $\mathrm{MeV}$ or more, predominantly lose energy via the process of bremsstrahlung. High energy photons, in the vicinity of an external electromagnetic field, will convert to a an electron positron pair (pair production). The photon emitted by bremsstrahlung will undergo pair production and the electron positron pair will emit bremsstrahlung, creating a shower of particles. This will continue until the electrons and photons cease to have enough energy to carry on showering. These low energy particles will ionise the active material of the calorimeter and this charge can be detected. The amount of charge is proportional to the number of particles in the shower which is proportional to the energy of the incoming particle. The constant of proportionality has to be empirically determined using electronic signals of known strength and standard physics signals such as $\mathrm{Z} \rightarrow e^{+} e^{-}$.

Charged hadrons do emit bremsstrahlung, but, as the rate of emission is roughly inversely proportional to the mass of the particle squared, they lose energy to bremsstrahlung a lot more slowly than electrons. Neutral hadrons, such as neutrons, do not emit bremsstrahlung at all. The predominant manner in which hadrons lose energy is through ionisation and via nuclear interactions. Hadronic showers are created when a high energy hadron interacts with one of the nuclei in the calorimeter 
to produce a series of hadrons. These secondary particles will then produce more particles, in an analogous way to an electromagnetic shower, until all particles cease to have enough energy to produce any new particles. These low energy particles will then deposit their energy via ionisation, which can be collected and recorded. One important feature to note about hadronic showers is that the characteristic length of a hadronic shower is about an order of magnitude larger than that of an electromagnetic shower.

Apart from neutrinos there is one type of particle that will traverse the calorimeter, muons. Muons are too massive to emit much bremsstrahlung and do not interact via the strong force. Therefore they undergo neither an electromagnetic nor a hadronic shower. The only manner in which they interact is through ionising the liquid argon as they pass through it. A muon will deposit roughly $2.5 \mathrm{GeV}$ in the $\mathrm{D} \varnothing$ calorimeter through ionisation. Because, in the energy ranges that they are produced in $\mathrm{D} \varnothing$, muons only produce the minimum amount of ionisation they are known as minimum ionising particles (MIPs).

\section{The Pre-Shower Detectors}

The first section of the calorimeter encountered traveling out from the central tracking system are the pre-shower detectors. These combine the principles of tracking and calorimetry to enhance the ability of the $\mathrm{D} \varnothing$ detector to discriminate between different types of particles. The two components of the pre-shower are a heavy material to induce showering, especially of electrons and photons, and scintillators to detect the products of these showers. In the central region $(|\eta|<1.2)$ the solenoid magnet acts to induce showering, so only a single layer of lead is added along with

three layers of scintillators. In the forward region $(1.4<|\eta|<2.5)$, where there is no solenoid to induce showering, there are three layers of lead to induce showering and three layers of scintillators. 


\section{The Calorimeter}

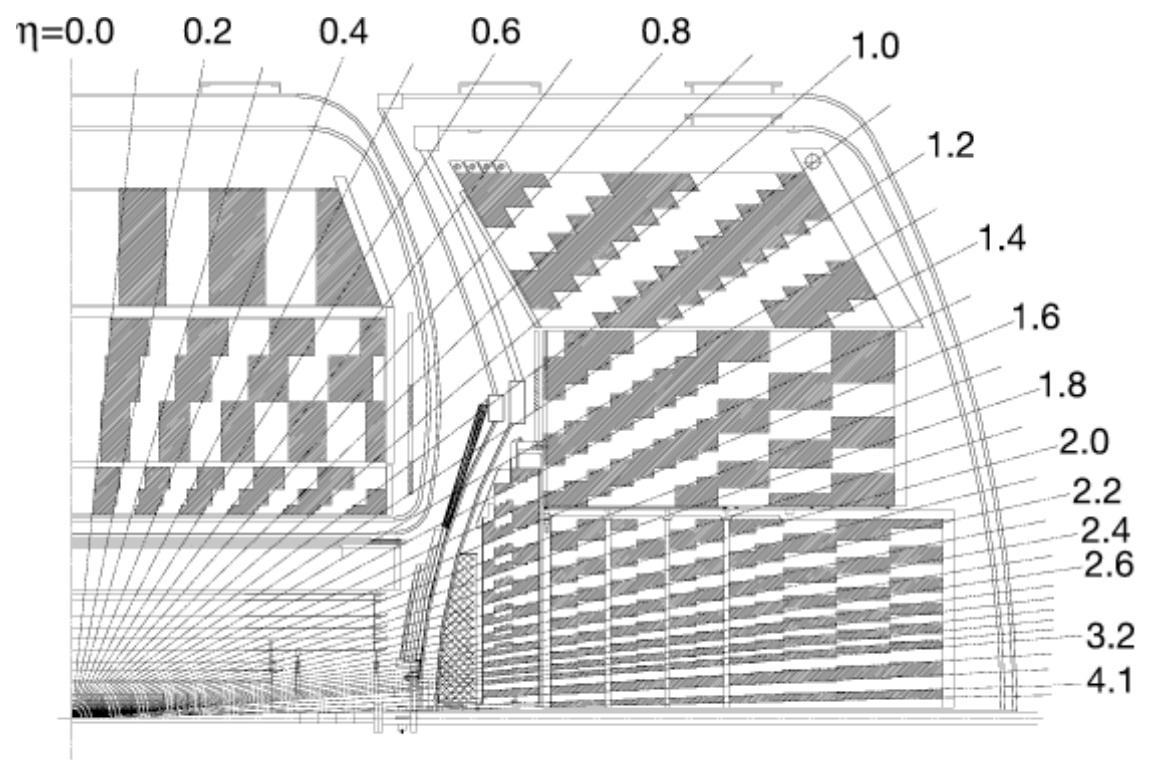

Figure 3.6: A segmented view of a quarter of the calorimeter.

The DØ calorimeter, which lies beyond the pre-shower detectors, is a sampling

calorimeter. A sampling calorimeter consists of two types of material, an absorber to induce the showers and an active material that is used to collect the ionised charge produced in the showers. The calorimeter is split into three modules, a cylindrical central calorimeter, and two disc shaped end calorimeters. Within each of these modules the calorimeter is segmented into cells, which themselves are arranged into layers. This arrangement can be seen in Figure 3.6, which shows a cross section of a quarter of the calorimeter. Each cell is constituted of layers of absorber, in most of the calorimeter uranium, and layers of active material, liquid argon. The ionised charge from the argon is detected using copper plates located within the cell.

The first four layers in the calorimeter contain most electromagnetic showers and so are collectively described as the electromagnetic calorimeter. The absorbing material in these layers consists of 3-4 mm layers of uranium. The segmentation 
of the cells in $\eta \times \phi$ is $0.1 \times 0.1 \mathrm{rad}$ with the exception of the third layer, where most the energy from electromagnetic showers is expected to be deposited, where the segmentation is $0.05 \times 0.05 \mathrm{rad}$.

The next three layers constitute the fine hadronic calorimeter, where most of the energy from hadronic showers is deposited. The absorbing material consists of thicker, $6 \mathrm{~mm}$, layers of uranium. Any particles that pass through the fine hadronic calorimeter enter the coarse hadronic calorimeter. It is one layer thick in the central region and up to three layers thick in the forward region. The absorbing material consists of $46.5 \mathrm{~mm}$ plates of copper or stainless steel. The segmentation of the cells in the hadronic calorimeter in $\eta \times \phi$ is $0.1 \times 0.1 \mathrm{rad}$.

\subsubsection{The Muon Detectors}

In general the only particles, except neutrinos, that can pass through the calorimeter without losing all their energy through showering are muons, which emit little bremsstrahlung and do not interact via the strong force.

The muon system, shown in Figure 3.7, consists of three subsystems

- Drift chambers

- Scintillation counters

- A $1.8 \mathrm{~T}$ toroidal magnet.

The drift chambers and scintillation counters are arranged into three layers which, traveling from the beampipe, are denoted the $A, B$ and $C$ layers. Between the $\mathrm{A}$ and $\mathrm{B}$ layers is the toroidal magnet, which allows a measurement of the $p_{t}$ of the muon to be made. Each layer consists of a plane of scintillation counters and a plane of drift chambers. The scintillation counters provide a fast signal that 


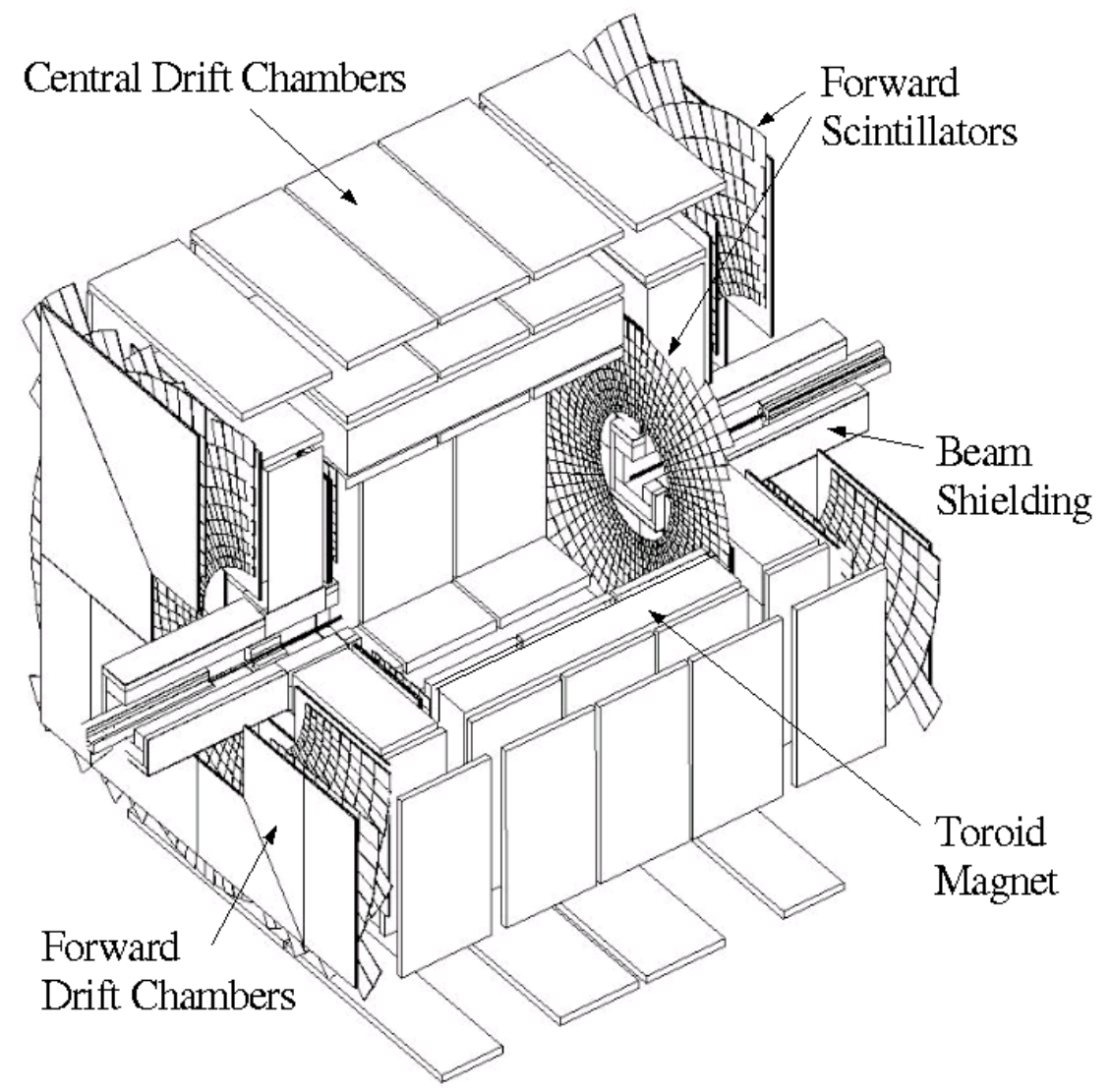

Figure 3.7: The muon system [23].

can be associated with a given bunch crossing. This is useful in triggering and in discriminating against cosmic ray backgrounds. The drift counters provide a more accurate measurement of position.

The muon system is split into two regions, the forward system (the FAMUs) and the central system (the WAmus). The Wamus, lying within $|\eta|<1$, consists of planes in $x$ and $y$. The FAMUs, lying with $1<|\eta|<2$ consists of planes in $z$. There is also a region, at the bottom of the detector, where the coverage of the muon system is compromised by the calorimeter supports and electronics as can be seen in Figure 3.2. This region lies within $|\eta|<1.25$ and between $4.25<\phi<5.15$. 
The drift chambers are made up of a series of cells, each filled with a gas that is ionised if a charged particle passes through it. The electrons are collected by an anode wire that passes along the centre of the cell. The time that the signal reaches either end of the wire allows a measurement of the position along the wire where the particle passed. The distance that the particle passes from the wire can be calculated using the time taken for the electrons to reach the wire. The cells used in the WAMUS and the FAMUS are different due to the presence of a higher amount of background in the forward region.

The cells in the wamus are Proportional Drift Tubes (PDTs). Each PDT is an aluminium cell of cross section $6 \times 10 \mathrm{~cm}^{2}$ and of varying lengths, up to $6 \mathrm{~m}$. Each is filled with a gas that is $84 \%$ argon, $8 \%$ methane and $8 \%$ carbon tetra-fluoride. The drift distance resolution is around a millimetre. The resolution along the wire depends on where the muon passes through the chamber. If the muon passes close to the centre of the cell, the resolution is around $10 \mathrm{~cm}$, if the muon passes close to the end of the cell, the signal has to travel further and dispersion causes the resolution to degrade to around $50 \mathrm{~cm}$. Typical modules are 24 chambers wide and contain 72 or 96 PDTs. The A layer consists of 4 decks of PDTs except the bottom layer which has three decks. The other layers each consist of three decks of PDTs.

The cells in the FAmus are Mini Drift Tubes (MDTs). Each MDT is an aluminium cell of cross section $9.4 \times 9.4 \mathrm{~mm}^{2}$ and of varying lengths, up to $5.83 \mathrm{~m}$. Each is filled with a gas that is $90 \% \mathrm{CF}_{4}$ and $10 \%$ methane. The resolution of the distance of the muon to the anode is $0.7 \mathrm{~mm}$, with no attempt to determine the distance along the wire. All three layers consist off three decks of MDTs. Within these decks the MDTs are grouped into octants, as can be seen in Figure 3.7.

Photons produced in the scintillator are collected using photo-multiplier tubes, PMTs, which convert the light to electronic signals. This signal is digitised to produce an accurate measurement of the time that the muon passes through the system, with a resolution of around 2 ns, and the position. There are tiers of 
scintillators in all three layers of the FAMUS and in the A and C layers of the wAMUS.

\subsubsection{The Luminosity Monitors}

The luminosity of any given data sample is obtained by normalising to the total $\mathrm{p} \overline{\mathrm{p}}$ inelastic cross section. The total inelastic cross section is dominated by soft interactions, where little energy is transferred between colliding partons and no high $p_{t}$ particles are observed in the final states. Hard interactions, in addition to high $p_{t}$ particles, contain a soft underlying event produced by the proton and antiproton breaking up and interacting softly with each other. Therefore all events produce low $p_{t}$ particles, exiting the interaction region close to the beampipe.

To detect these particles the two luminosity monitors are situated in the far forward region of the detector, at $z= \pm 140 \mathrm{~cm}$, covering $2.4<|\eta|<4.4$. Each monitor consists of 24 wedges of scintillator. The frequency of hits in the scintillator can be used to calculate the luminosity, as described in more detail in Section 6.4.

\subsection{The DØ Trigger System}

In order to produce enough of the rare events that we are interested in, the total rate at which $\mathrm{p} \overline{\mathrm{p}}$ collisions occur is too high to be able to store all produced events. Therefore decisions must be made in real time about which events are interesting enough to be stored. This is done using a three tier event trigger system, which takes events at a rate of $1.8 \mathrm{MHz}$ and writes events to tape at a rate of around $50 \mathrm{~Hz}$. Each tier combines information from the subdetector systems to select some fraction of the events passed to it from the previous tier. 


\subsubsection{The Level 1 Trigger}

The first tier of the trigger system is based upon a specialised fast readout system to reduce the event rate from approximately $1.8 \mathrm{MHz}$ to approximately $2 \mathrm{KHz}$. It takes approximate information from all subdetectors, except the SMT, which it uses to make a decision about whether an event is passed. This trigger can either be based upon a single trigger object or upon some combination of these objects.

The fibre tracker uses hits in the axial fibres to provide a track trigger. Hits in each section are matched to a set of pre-defined hit maps, associated to tracks of different curvatures. The calorimeter trigger is based upon an approximate energy measurement of a tower of cells in the calorimeter. There is a separate trigger using just the electromagnetic calorimeter used in electron triggers. There are four programmable thresholds for each of these triggers.

The Level 1 muon trigger can use information from both the scintillator counters and the drift chambers (wires). The basic Level 1 requirement for muons is known as the tight scintillator requirement and requires that there are hits in both the A and $\mathrm{BC}$ layers of the muon system. This condition can be tightened by the addition of the loose wire requirement, which also requires that there is a hit in the drift chambers associated to these scintillator hits.

The fast-z trigger requirement requires a coincidence of hits in the luminosity monitors. This coincidence is associated with the remnants of protons that have been involved with an inelastic collision.

\subsubsection{The Level 2 Trigger}

The second tier of the trigger uses a similar fast readout to the first stage to reduce the event rate to approximately $1 \mathrm{KHz}$. The track trigger combines information from the SMT and the CFT to give an improved measurement of the $p_{t}$ of a track 
as well as information on the vertex position of the track. The calorimeter triggers are split into processors designed to find jets, electromagnetic objects (electrons and

photons) and missing energy. The muon triggers combine information from the drift chambers and the scintillators to obtain a track fit. A decision is made based upon the $p_{t}$ of this track and on the quality of the track fit. Loose muon objects require hits either inside or outside the toroid, medium objects require hits both within and without the toroid.

\subsubsection{The Level 3 Trigger}

The third layer of the trigger reduces the event rate to around $50 \mathrm{~Hz}$ at which stage it is stored to tape. Level 3 uses the full event readout, which it processes using a simplified version of the $\mathrm{D} \varnothing$ reconstruction code.

\subsection{Event Reconstruction at DØ}

If an event meets the trigger requirements the information from the detectors is read out as digitised information onto storage tapes. The reconstruction of the trajectories and energies of particles from this information proceeds in three stages.

- Hit finding; the digitised information is mapped to hits at definite positions in the detector at definite energies.

- Track and cluster forming; hits are assembled into either tracks in the central tracker or clusters in the calorimeter.

- Creation of physics objects; the tracks and clusters are grouped together to form physics objects, such as particles or jets. 


\subsubsection{Central Track Reconstruction}

The tracks of charged particles through the central tracker are reconstructed from hits in the CFT and SMT. The first stage is to form a hypothesis for a track from three hits in either the SMT or the CFT. This hypothesis is then extended outward and an expected crossing region formed. If a hit is discovered in this region it is added to the hypothesis, else a miss is attributed to it. A hypothesis is scrapped if the number of misses with respect to the number of hits is too high. The remaining hypotheses are ordered according to the number of hits, misses and the $\chi^{2}$ of the track fit. The hits associated with the track are divided into two categories; those that are associated only with that track and those that are shared with other tracks. If the ratio of the former to the latter is below a certain value then the track is stored.

The track is stored in the form of 5 parameters that define its trajectory. These parameters are

- $\frac{q}{p_{T}}$ : evaluated from the curvature of the track.

- $\phi_{0}$ : the angle between the track and the $x$ axis at the point of closest approach.

- $\eta_{0}$ : the 'physics' rapidity of the track measured at its origin.

- $z_{0}$ : the distance along the beam axis of the track at the point of closest approach.

- dca: the distance of closest approach of the track to the beam spot position in the $r \phi$ plane.

\subsubsection{Vertex and Beamspot Reconstruction}

The vertex finding algorithm utilises all reconstructed tracks in an event to determine the position of the inelastic $\mathrm{p} \overline{\mathrm{p}}$ collision. The first step is to fit all the tracks to a 
common vertex. If the value of the $\chi^{2}$ per degree of freedom is greater than ten the track making the greatest contribution to the $\chi^{2}$ is removed from the fit. This process is repeated until either the $\chi^{2}$ is less than ten or there is only one track remaining. The remaining tracks are then collected together and the process repeated on them.

The exact position of the beamspot is not a constant and so it is to be determined on a run by run basis to account for this variation over time. A run is a discrete period of detector operations lasting a few hours. The position of the beamspot is obtained by taking a linear average of all the vertices within a run.

\subsubsection{Calorimeter Object Reconstruction}

There are two types of calorimeter objects, electromagnetic and hadronic, distinguished by the distribution of energy within the calorimeter. Electromagnetic objects, electrons and photons, deposit energy mainly in the electromagnetic calorimeter. Hadronic objects deposit energy mainly in the hadronic calorimeter. Each kind of object is formed by grouping together energy deposits in neighbouring cells. A jet is produced by clustering several calorimeter objects together.

\subsubsection{Muon Reconstruction}

For the purposes of reconstructing the path of the muon, the muon system is divided into two regions. These are the $\mathrm{A}$ layer and the $\mathrm{B}$ and $\mathrm{C}$ layers (the $\mathrm{BC}$ layer). The first stage is to construct segments in each of these regions. A segment is formed from a straight line fit from two or more hits in the drift chambers. After this has been done any nearby hits in the scintillators can be added.

A track is formed by combining segments from the two regions. The amount that the muon track has been bent can be used to provide a measurement of the $p_{t}$ of the muon. The segments and tracks found in this way are known as local 
quantities. Central tracks are extrapolated through the detector to the muon system and attempted to match to each local track. If this match is successful, the $p_{t}$ of the muon is taken from the central track due to the superior resolution of the tracker. Even though the muon system $p_{t}$ resolution is inferior to that of the central tracker it still has many uses such as triggering and separating signal from background.

An important local quantity relates to the quality of the local track. A local track can either be described as tight, medium, loose or fail to pass the quality requirements at all. Progressing from loose to tight quality a track must pass ever stricter quality requirements. These requirements include the presence of segments in different layers of the detector, whether the muon is matched to a track in the central detector amongst others. A detailed description of the conditions required to meet the various quality requirements can be found in [24].

\subsubsection{Detection of Neutrinos}

Neutrinos interact so weakly that they are effectively never observed at D $\varnothing$. Instead they have to be inferred from the fact that there is an imbalance in the transverse momentum. This imbalance is known as the missing transverse energy $\left(\mathbb{E}_{T}\right)$. The transverse energy is obtained using by summing up the transverse energy $\left(E_{\mathrm{T}}\right)$ in all the cells in the calorimeter. The transverse energy is defined in terms of the energy deposited in the cell $(E)$ and the polar angle of the cell with respect to the primary vertex:

$$
E_{\mathrm{T}}=E(\sin \theta)
$$

The missing transverse energy is obtained by summing the $E_{\mathrm{T}}$ of all the cells in the calorimeter. Electrons are included separately, with a $0.5 \times 0.5$ rad region in $\eta \times \phi$ in the calorimeter around an identified electron excluded from the sum of 
the calorimeter cells [25]. This leads to a slight bias in the calculation of the $\mathbb{E}_{T}$, in a direction opposite to the electron direction which needs to be accounted for when trying to extract physics from a measurement of the $E_{T}$. This correction is luminosity dependent as the energy deposited by the products of soft interactions per calorimeter cell increases with luminosity. For the samples used in this thesis an overall average correction of $1.78 \pm 0.01 \mathrm{GeV}$ per electron was sufficient [26]. A further correction has to be applied for muons, which deposit very little energy in the calorimeter. The procedure adopted is that the $p_{t}$ of the muon, as measured by the central tracking system, is included in the sum of transverse energy. 


\section{Chapter 4}

\section{Simulation of Events using Monte Carlo Generators}

The term Monte Carlo is used to cover all techniques that employ random numbers to solve numerical problems, the term originating from the famous Monegasque casino. The statistical element in quantum mechanics and the complexity of the processes studied in high energy physics invites the use of Monte Carlo techniques. The main use of these in high energy physics is to take a theoretical model and provide a prediction of what is expected to be observed in a detector. This process is split into two major components; the generation of events and the simulation of how these events will be observed by a detector. The first stage, described in Section 4.1, performed by an event generator, uses a theory, typically the Standard Model, to produce a series of events. The latter stage, described in Section 4.2, models the fact that what is observed in a detector depends on the composition and resolution of a given detector. The remainder of the chapter, Section 4.3, describes the specific simulation of the $\mathrm{D} \emptyset$ detector used in this analysis. 


\subsection{Event Generators}

The purpose of an event generator is to attempt to produce events in as much detail as could be seen using a perfect detector. This is done by dedicated programs such as PYTHIA [27] or HERWIG [28], which do not do this is a in a single step but factorise it out into a number of components, each of which can be modelled reasonably accurately.

The first step is to calculate the hard scatter, the process where two partons interact producing one or more fundamental particles. In hadron-hadron collisions this involves picking partons out of the hadron using parton distribution functions (PDFs). As the analysis described in this thesis is sensitive to the modelling of PDFs they are discussed in more detail in Chapter 7. As the hard scatter is at high energies it is typically only calculated to leading order (LO), neglecting the higher order terms ${ }^{1}$. However some of the latest generation of generators, such as MC@NLO [29], do include all terms up to NLO for a limited number of processes.

The next step is to model the initial and final state radiation from the fundamental particles in the event. This is done using a process known as parton showering. Parton showers use the assumption that all radiation can be factorised into a series of $1 \rightarrow 2$ processes. The shower starts off at the energy scale of the hard process and is evolved down to some cutoff point, when the process is stopped.

As quarks and gluons are not observed directly the final step is to gather them together into hadrons. This is done using non-perturbative models, such as the string model in PYTHIA or the cluster model in HERWIG. The final step is to take any unstable particles and decay them until all remaining particles are stable, within the timescales required.

\footnotetext{
${ }^{1}$ It is not completely true to call PYTHIA and HERWIG LO generators as the parton shower introduces an element of higher order corrections.
} 


\subsection{Detector Simulation}

In order to compare the processes produced by event generators with what is seen in the detector various effects must be taken into account. These include the effects of material and magnetic fields on the particles, noise in the detector and the position and energy resolution of the detector. At D $\varnothing$ there are two approaches to modelling these effects.

The first approach is taken by the DØgstar package [30], which shall now be referred to as DMCS. This uses the CERN package GEANT (Version 3) [31] to build up a detailed model of the detector with information on how each material will interact with a given particle. The output of this package is in the same format as the data and can be reconstructed and analysed using the same code. However this approach is time consuming and the complexity of the detector makes reproducing what is seen in data difficult.

The other approach is to parameterise the effects of the detector resolution on particles and physics objects, such as jets and muons. This parameterisation is then tuned to give agreement between the data and the simulation. This is done using the PMCS program [32], which is described in more detail in Section 4.3. The advantage of PMCS is that it is much quicker to produce events than DMCS, allowing the production of very large samples.

\subsection{PMCS}

The PMCS program is used to model the effects of the D $\varnothing$ detector on generated events. It does this by parameterising the total effect of detector resolution on physics objects. PMCS consists of several sub-programs, each simulating the effect of detector resolution on a given type of particle or physics object. The sub-packages 
model:

- The track resolution and efficiency (Section 4.3.1).

- The muon system resolution and reconstruction and trigger efficiencies (Section 4.3.3).

- The effect of the calorimeter on electrons, photons and jets (Section 4.3.4).

- The missing energy resolution (Section 4.3.5).

- A simple trigger system (Section 4.3.6).

\subsubsection{Track Resolution}

The simulation of the $p_{t}$ resolution is done in two stages using three variable parameters, denoted $A, B$ and $C[33,34]$. Firstly the generated $p_{t}$ is smeared using a Gaussian distribution with a mean at the generated $p_{t}$ and a width given by Equation 4.1 to give a partially smeared momentum, $p_{t}^{\prime}$. To obtain the final smeared momentum $\left(p_{t}(\right.$ smear $\left.)\right), p_{t}^{\prime}$ is scaled using Equation 4.2 .

$$
\begin{gathered}
\frac{\sigma_{1 / p_{t}}}{1 / p_{t}}=\sqrt{A^{2} \frac{p_{t}^{2}}{L^{4}}+\frac{B^{2}}{L \sin (\theta)}} \\
p_{t}(\text { smear })=C p_{t}^{\prime}
\end{gathered}
$$

Here $L$ is the track bending lever arm, defined as;

$$
L= \begin{cases}1, & \text { if }\left|\eta_{\text {phys }}\right|<1.62 \\ \frac{\tan \left(\theta_{\text {phys }}\right)}{\tan \left(\theta_{C F T}\right)}, & \text { otherwise. }\end{cases}
$$


The parameter $A$ describes the effect on the $p_{t}$ resolution caused by the finite resolution of individual hits in the tracker. As the tracks get straighter with increasing $p_{t}$ the fractional error on the curvature, and hence on the $p_{t}$, increases. This results in the resolution degrading as $p_{t}$ increases.

The lever arm refers to the distance, in the plane perpendicular to the magnetic field, from the vertex to the point where the track exits the tracker. The $p_{t}$ resolution becomes poorer as this distance decreases. The model used is a simplified one that accounts for the fact that at high $\theta_{C F T}$, as the $\theta_{C F T}$ of the track increases, the number of the layers of the CFT it passes through decreases.

$B$ parameterises the effect of multiple scattering on the resolution. $C$ parameterises the imperfect description in the reconstruction process of the magnetic field and the energy loss caused by passing through material. The values of $A, B$ and $C$ are obtained by optimising the agreement between the PMCS simulation and the data. This procedure is described in Section 8.1, along with the parameters obtained.

\subsubsection{Addition of Tracking Efficiencies}

The high multiplicity of particles and the rapid rate of collisions, with the associated problems of pileup and noise, make modelling of efficiencies from first principles difficult. Therefore tracking efficiencies are evaluated from the data, using the tag and probe method.

This method 'tags' an event from some well understood physics process, such as $\mathrm{Z} / \gamma^{*} \rightarrow \mu^{+} \mu^{-}$, using all the criteria except the one we are interested in. The efficiency is determined by 'probing' whether the requirement that we are interested in, such as whether there is a reconstructed track, is met. As the processes considered here deal with high $p_{t}$ muons then all the efficiencies are evaluated using $\mathrm{Z} / \gamma^{*} \rightarrow \mu^{+} \mu^{-}$events. The event is selected by requiring two muons identified by 


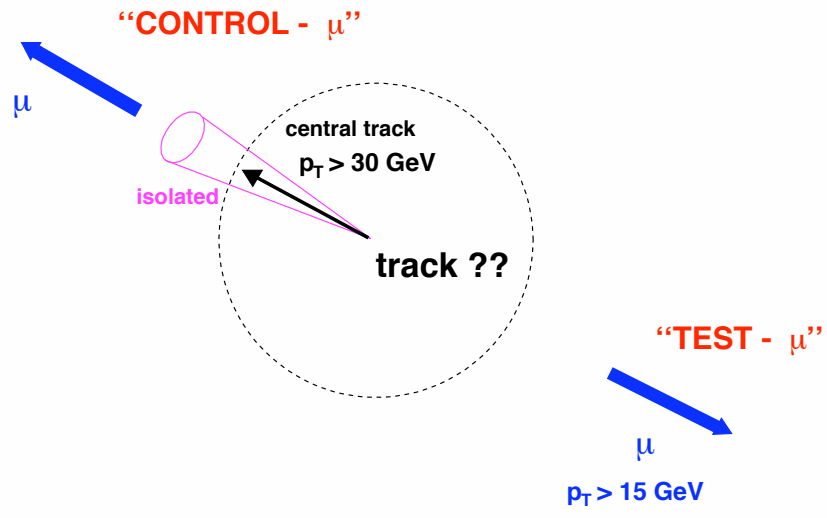

Figure 4.1: Diagram of tag and probe method used to evaluate tracking efficiency.

the muon system, one of which is matched to a track in the central detector. The efficiency is then the fraction of times that the second muon has a track matched to it. A diagram illustrating this procedure is shown in Figure 4.1. A fuller description, with detailed discussion of problems with backgrounds and a full list of the slight differences in selection cuts used to minimise these problems can be found in [35] and [36].

The efficiencies are introduced into the simulation as a function of the detector geometry in such a way as to minimise any bias. For each track two random numbers are used to determine whether the track is reconstructed or not and whether the track is detected by Level 3 of the trigger. PMCS then has a simple Boolean output, the track is either found by the event reconstruction or not and the track is detected by the trigger or not. The modelling of these efficiencies is described in more detail in Section 8.2. 


\subsubsection{Muon Resolution and Efficiencies}

\section{Local Muon System Resolution}

The local muon system $p_{t}$ resolution is studied using the variable mures [37], defined as

$$
\text { mures }=\frac{\sigma(q / p)}{q / p}=\left[\frac{q / p(\text { local })-q / p(\text { cen })}{q / p(\text { cen })}\right]=\frac{\sigma(p)}{p} .
$$

Where local refers to the quantities measured in the muon chambers and cen refers to quantities measured by the central tracking system. The functional form of the momentum resolution (in $\mathrm{GeV}$ ) was parameterised as

$$
\frac{\sigma(p)}{p}=\frac{\alpha(p-\beta)}{p} \oplus \gamma p
$$

where the parameters $\alpha, \beta$ and $\gamma$ are used to describe the effects of multiple scattering, energy loss by the muon and finite position resolution respectively ${ }^{2}$. Firstly the momentum has an amount, $\beta \mathrm{GeV}$, subtracted off to represent the energy lost by the muon before it reaches the muon system, then this momentum is smeared using a Gaussian distribution with a width given by Equation 4.5. The values of the parameters were set, separately for the WAMUS and the FAMUs by fitting to a sample of di-muon events. The values are listed in Table 4.1 [38].

\begin{tabular}{||l|l|l|l|}
\hline Region & $\alpha$ & $\beta(G e V)$ & $\gamma\left(\mathrm{GeV}^{-1} 1\right)$ \\
\hline \hline WAMUS & $0.362 \pm 0.038$ & $3.09 \pm 0.20$ & $0.0314 \pm 0.0030$ \\
FAMUS & $0.210 \pm 0.010$ & $1.79 \pm 0.16$ & $0.0058 \pm 0.0005$ \\
\hline \hline
\end{tabular}

Table 4.1: Parameters used in local muon $p_{t}$ resolution.

\footnotetext{
${ }^{2}$ The symbol $\oplus$ indicates that the two terms are added in quadrature.
} 


\section{Muon System Efficiencies}

The muon efficiencies are also measured using $\mathrm{Z} \rightarrow \mu^{+} \mu^{-}$data with the tag and probe method. The procedure used to measure the efficiencies is similar to that used to measure the tracking efficiencies, except that the event is now tagged using two tracks and one muon and the muon identification efficiency is the fraction of times that the other muon is found. Included are the muon identification efficiencies and the efficiencies for the various objects of the muon triggers, as defined in Section 3.3. These include the probability that the tight scintillator and loose wire requirements are met and that the muon is identified as being of at least medium quality at Level 2 . The modelling of these efficiencies is described in more detail in Section 8.2. PMCS produces the same Boolean output for each of these efficiencies, with this result being produced using random numbers in the same way as for the tracking efficiency.

\subsubsection{Electron, Photon and Jet Resolution}

PMCS models the effect of detector resolution separately for electromagnetic objects, photons and electrons, and hadronic objects, jets.

\section{Electromagnetic Object Resolution and Efficiencies}

Electromagnetic objects is the collective name for electrons and photons, being the particles that interact mainly with the electromagnetic calorimeter. At present the energy and angular resolution for electrons and photons are treated in the same way. This model is based upon studies of electrons, in particular $\mathrm{Z} \rightarrow e^{+} e^{-}$, so may not be completely correct for photons. However, as seen by the calorimeter, electrons and photons are similar enough to make this assumption reasonable at the level of accuracy required by this analysis.

The modelling of the electromagnetic resolution proceeds in two stages using 
parameters [26]. Firstly the generated energy is scaled, to account for imperfection in the calibration, using Equation 4.6. Here $\alpha$ and $\beta$ are variable parameters representing the energy scale and offset respectively:

$$
E^{\prime}=\alpha E_{g e n}+\beta
$$

The energy, $E^{\prime}$, is smeared using a Gaussian with width given by Equation 4.7. Here $C$ and $S$ are parameters representing the constant and sampling terms respectively:

$$
\frac{\sigma_{E^{\prime}}}{E^{\prime}}=\sqrt{C^{2}+\frac{S^{2}}{E^{\prime}}} .
$$

The parameters used are different in the central and forward calorimeters, to reflect the different construction of these sub-detectors.

The angular resolution of the calorimeter is modelled by smearing the $\eta_{\text {det }}$ and $\phi_{\text {det }}$ using Gaussians centered at the generated value of each variable and with widths, $\sigma_{\eta_{\text {det }}}$ and $\sigma_{\phi_{\text {det }}}$, obtained by optimising the agreement of the angular resolution between $\mathrm{Z} \rightarrow e^{+} e^{-}$data and the PMCS simulation. The electron identification efficiencies are measured using $\mathrm{Z} \rightarrow e^{+} e^{-}$data in an analogous way to the muon efficiencies. A full description of the choice of variables used to limit biases can be found in [39].

\section{Jet Resolution}

At present PMCs does not model any jet finding efficiency, with a jet passing through the fiducial volume of the calorimeter automatically assumed to be detected. The first step taken by PMCS is to propagate all the generated particles out to the calorimeter. These particles are then clustered into jets using the cone algorithm [40], ignoring any detector effects on the individual particle. The effects of detector resolution are modelled using the jets themselves [32]. 
The first stage is to scale the generated energy of the jet using Equation 4.8 to account for imperfect calibration and for the fact that some of the energy will be deposited in uninstrumented parts of the calorimeter:

$$
E_{j e t}^{\prime}=\alpha_{j e t} E_{j e t}^{g e n}+\beta_{j e t}
$$

Then this energy is smeared using a Gaussian with width given by Equation 4.9 and mean at the scaled energy. Here $C_{j e t}, S_{j e t}$ and $N_{j e t}$ are variable parameters representing the constant, sampling and noise terms respectively.

$$
\frac{\sigma_{E}}{E_{j e t}}=\sqrt{C_{j e t}^{2}+\frac{S_{j e t}^{2}}{E}+\frac{N_{j e t}^{2}}{E^{2}}} .
$$

Although the form of the parameterisation is similar to that used for electrons the values of the parameters are, in general, different. This is due to the different distribution within the calorimeter of the energy deposited by jets and electrons. The constants are determined separately for the different $\eta$ ranges, to reflect the variation of the detector. For a detailed description of the parameterisation see [41].

\subsubsection{Missing Energy Resolution}

PMCs has two approaches to modelling the missing energy resolution, the first uses the jets in an event. This model performs a vector sum of the jets before and after the smearing is performed. The smeared sum is subtracted from the unsmeared sum and, after accounting for leptons, the missing energy is obtained.

The second model, used only in electroweak analyses including the analysis dis-

cussed in this thesis, does not use the final state jets to evaluate the $\mathbb{E}_{T}$. The method assumes that the only hard jets produced are recoiling from the produced electroweak boson. The $E_{T}$ resolution is parameterised using the recoil of the pro- 


\begin{tabular}{||l|l|}
\hline Parameter & Value with Uncertainty \\
\hline$\alpha$ & $0.60 \pm 0.02$ \\
$\mathrm{~S}\left(\mathrm{GeV}^{\frac{1}{4}}\right)$ & $0.80 \pm 0.20$ \\
$\mathrm{C}$ & $0.05 \pm 0.01$ \\
$\mathrm{U}(\mathrm{GeV})$ & $3.02 \pm 0.04$ \\
\hline
\end{tabular}

Table 4.2: Summary of parameters used to smear $\mathbb{E}_{T}$

duced boson, the smeared lepton $p_{t}$, the neutrino $p_{t}$ and a term that models the soft underlying event. The resolution of the recoil jet is evaluated in a similar manner to other jets, using a two stage process. Firstly the energy of the jet is scaled by the hadronic energy scale, $\alpha_{\text {had }}$ :

$$
E_{\mathrm{T}_{\text {recoil }}^{\prime}}^{\prime}=\alpha_{\text {had }} E_{\text {Trecoil }} .
$$

This energy is then smeared using a Gaussian with a width given by equation 4.11 and a mean at the scaled energy.

$$
\frac{\sigma_{E_{\mathrm{T}_{\text {recoil }}^{\prime}}^{\prime}}}{E_{\mathrm{T}_{\text {recoil }}^{\prime}}}=\sqrt{C_{\text {had }}^{2}+\frac{S_{\text {had }}^{2}}{E_{\mathrm{T}_{\text {recoil }}^{\prime}}^{\prime}}},
$$

where $C_{\text {had }}$ and $S_{\text {had }}$ are the constant and sampling terms from the hadronic calorimeter.

The underlying event is simulated using a vector with random direction and a magnitude randomly distributed according to a Gaussian of mean zero and width $U$. The parameters are obtained, for a given data taking period, by optimising the agreement between $\mathrm{Z} \rightarrow e^{+} e^{-}$data from that data taking period and a PMCS simulation of $\mathrm{Z} \rightarrow e^{+} e^{-}$events [39]. These parameters are summarised in Table 4.2.

The original model assumed that muons pass through the calorimeter without interacting. This led to a discrepancy between the transverse mass distribution 
in $\mathrm{W} \rightarrow \mu \nu$ events in data and PMCS. To correct this discrepancy a model for energy deposition by a minimally ionising particle (mip) such as a muon was added to the simulation. This model uses a simple model of the calorimeter, containing information on the thickness and material composition of the calorimeter at a given point, to model the energy deposition as a function of $\eta$ and $\phi[42]$.

To get the transverse component of this the energy is divided by $\cosh (\eta)$. The quantity is then multiplied by a parameter, $k$, which represents a 'mip' energy scale. The value of $k$ was determined be $0.86 \pm 0.21$ by optimising the agreement between data and PMCS using $\mathrm{W} \rightarrow \mu \nu$ data [42].

The smeared $\mathbf{E}_{\mathbf{T}}$ is then calculated using the following vector sum

$$
\mathbf{E}_{\mathbf{T}}=-\mathbf{p}_{\mathbf{t}}{ }^{\mu}-\mathbf{E}_{\mathbf{T}}{ }^{\text {recoil }}-\mathbf{E}_{\mathbf{T}}{ }^{\text {ue }}-\mathbf{E}_{\mathbf{T}}{ }^{\text {mip }}
$$

where $\mathbf{p}_{\mathbf{t}}{ }^{\mu}$ is the smeared $p_{t}$ of the muon, $\mathbf{E}_{\mathbf{T}}{ }^{\text {recoil }}$ is the smeared $E_{\mathrm{T}}$ of the recoil jet, $\mathbf{E}_{\mathbf{T}}{ }^{\text {ue }}$ is the smeared $E_{\mathrm{T}}$ of the underlying event and $\mathbf{E}_{\mathbf{T}}{ }^{\text {mip }}$ is the transverse energy deposited any muons in the calorimeter.

\subsubsection{Simulation of Triggers}

Each trigger at $\mathrm{D} \varnothing$ is made up of a series of trigger objects, such as the Level 1 muon scintillator trigger and the Level 3 track trigger. Whether an overall trigger requirement is met is determined by whether the trigger objects making up the trigger requirement are met. Whether these objects are found is determined by the pertinent subprogram, i.e. whether the track trigger requirement is met is determined by the tracking subprogram. The role of the trigger subprogram is merely to collate these objects and determine whether overall trigger requirements are met. The only triggers at present available are the di-muon and single muon triggers used in the $\mathrm{Z} \rightarrow \mu^{+} \mu^{-}$and $\mathrm{W} \rightarrow \mu \nu$ analyses. A detailed description of the implementation of 
these triggers can be found in Section 8.2. 


\section{Chapter 5}

\section{Electroweak Physics at the Tevatron}

This chapter gives a more detailed description of the area of particle physics relevant to this thesis; that is electroweak physics at the Tevatron. In this thesis, electroweak physics refers to processes that involve two of the electroweak bosons, the $\mathrm{W}$ and the $\mathrm{Z}$.

The chapter commences with a description of how the electroweak bosons are produced at the Tevatron and how they decay. It digresses to discuss certain properties of the bosons, such as invariant mass and width, before continuing with a description of measurements that can be performed using electroweak bosons. The chapter concludes with a discussion of the measurement made in the thesis, that of the indirect measurement of the width of the $\mathrm{W}$ boson. 


\subsection{Production and Decay Mechanisms}

At the Tevatron electroweak bosons are predominantly produced by quark-antiquark fusion. The bosons rapidly decay to a pair of fermions. The decay channels are listed in Table 5.1 and 5.2. An example of the production and decay of a $\mathrm{Z}$ boson is shown in Figure 5.1.

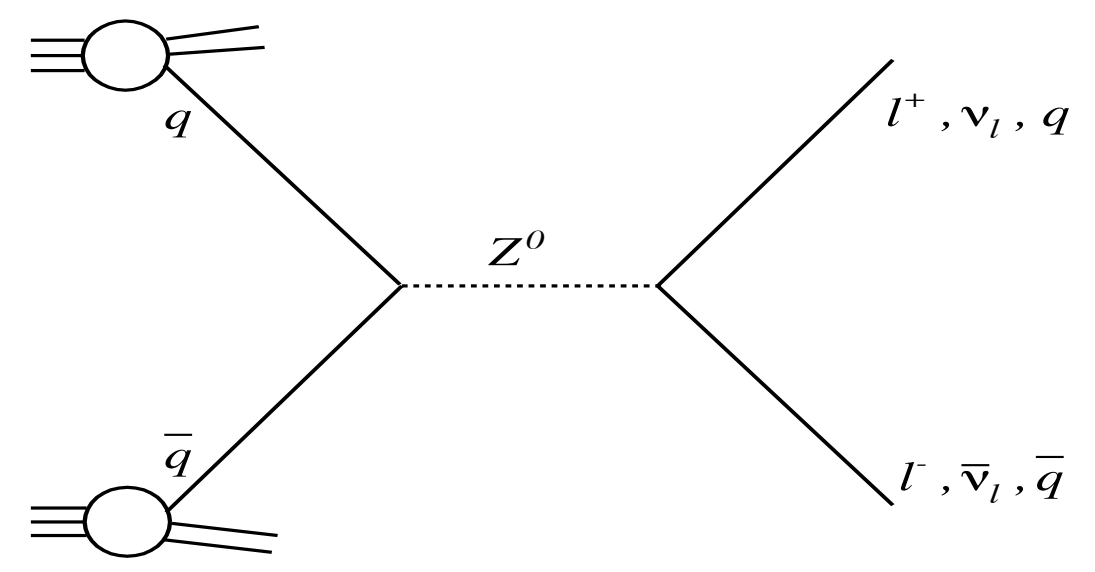

Figure 5.1: Diagram of $\mathrm{Z}$ boson production from quark-antiquark fusion.

\begin{tabular}{|c|c|}
\hline Decay Channel & Branching Fraction (\%) \\
\hline$e \nu_{e}$ & $10.72 \pm 0.16$ \\
$\mu \nu_{\mu}$ & $10.57 \pm 0.22$ \\
$\tau \nu_{\tau}$ & $10.74 \pm 0.27$ \\
hadrons & $67.96 \pm 0.35$ \\
\hline
\end{tabular}

Table 5.1: Branching fractions of the decays of the $\mathrm{W}$ boson [2].

For both electroweak bosons the predominant decay channel is that to hadrons, but due to the large backgrounds from purely QCD processes it is hard to isolate these events. There are fewer background processes that produce high $p_{t}$ leptons, so it is easier to select a clean sample of electroweak bosons in these channels. A further advantage of using leptons is that it is much easier to make measurements 


\begin{tabular}{|c|c|}
\hline Decay Channel & Branching Fraction $(\%)$ \\
\hline$e^{+} e^{-}$ & $3.363 \pm 0.004$ \\
$\mu^{+} \mu^{-}$ & $3.366 \pm 0.007$ \\
$\tau^{+} \tau^{-}$ & $3.370 \pm 0.008$ \\
invisible & $20.00 \pm 0.06$ \\
hadrons & $69.91 \pm 0.06$ \\
\hline
\end{tabular}

Table 5.2: Branching fractions of the decays of the $\mathrm{Z}$ boson [2]

of their properties, such as $p_{t}$ or angular distributions. This makes it easier to study the kinematics of electroweak boson production and decay.

\subsubsection{Invariant Mass Distribution of the $\mathrm{Z}$ Boson}

The simplest example of one of these kinematic distributions is the mass of the $\mathrm{Z}$ boson, which is calculated from the momenta of the decay products as follows

$$
M_{Z}=\sqrt{\left(p_{1}+p_{2}\right)^{2}-\left(p_{x_{1}}+p_{x_{2}}\right)^{2}-\left(p_{y_{1}}+p_{y_{2}}\right)^{2}-\left(p_{z_{1}}+p_{z_{2}}\right)^{2}},
$$

where $p$ are the total momenta and $p_{x}, p_{y}$ and $p_{z}$ the components in the $x, y$ and $z$ directions respectively. The subscript numbers refer to the two different leptons. This assumes that the two leptons are massless, and can be re-expressed as

$$
M_{Z}=\sqrt{2 p_{1} p_{2}\left(1-\cos \phi_{12}\right)}
$$

where $\phi_{12}$ is the angular separation of the two muons.

The invariant mass distribution for events passing the $\mathrm{Z} \rightarrow \mu^{+} \mu^{-}$event selection at $\mathrm{D} \varnothing$, as described in Section 6.1.1, is shown in Figure 5.2. This distribution is not a $\delta$ function at the mass of the $\mathrm{Z}$ for various reasons. The first stems from Equation 2.1 


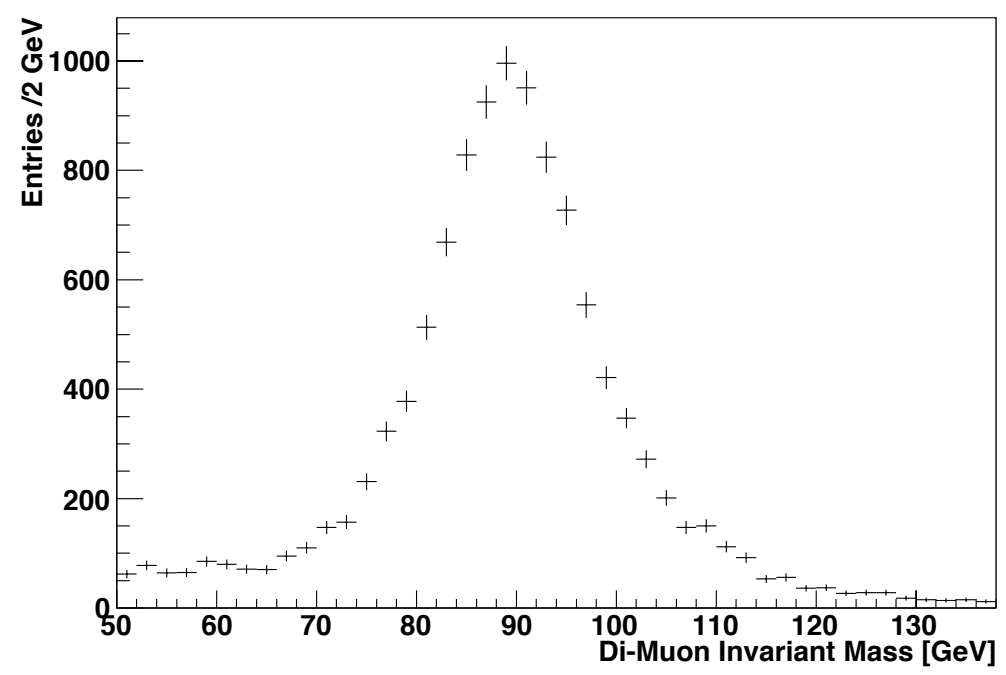

Figure 5.2: Invariant mass distribution of events passing the $\mathrm{Z} \rightarrow \mu^{+} \mu^{-}$event selection [35].

because, as the $\mathrm{Z}$ boson is a short lived state, it is produced with a range of masses. A second, much larger effect at $\mathrm{D} \varnothing$, is that the Energy and momentum resolution of the detector is not perfect, broadening the observed mass distribution. The last is that there is also some interference from the process $p \bar{p} \rightarrow \gamma^{*} \rightarrow \mu^{+} \mu^{-}$, which has a flatter invariant mass distribution. The photon contribution can be seen in the low mass 'shoulder' in Figure 5.2.

\subsubsection{Particle Widths}

The differential cross section, with respect to the centre of mass energy of the state, for the production of a resonance has the form of 


$$
\frac{d \sigma}{d M} \propto \frac{1}{(E-M)^{2}+(\Gamma / 2)^{2}}
$$

where $E$ is the centre of mass energy of the state, $M$ the mass of the resonance and $\Gamma$ is the width of the resonance [1]. The width relates to the lifetime of the resonant state $(\tau)$ according to Equation 2.1 with the width corresponding to $\Delta E$ and the lifetime to $\Delta t$. Therefore the width can be used as another way of expressing the lifetime of a particle, with long lived states, like the $J / \Psi$ having narrow widths and short lived states having large widths.

Most unstable particles have several different decay channels that contribute to their lifetime. These decay channels can be thought of in terms of partial widths. Partial widths are an alternative way of expressing the branching fraction to a given decay channel, with branching ratios $(B R)$ expressed as

$$
B R=\frac{\Gamma_{i}}{\Gamma}
$$

Therefore knowledge of the partial width and the branching fraction of a decay channel can allow a measurement of the overall width.

\subsubsection{Transverse Mass Distribution of the W Boson}

As one of the leptonic decay products of the $\mathrm{W}$ boson is always a neutrino it is not possible to reconstruct the invariant mass of the $\mathrm{W}$ in the same way as the $\mathrm{Z}$. The procedure that has to be adopted is to measure the distribution of the transverse mass, $M_{T}$, of the candidate events. $M_{T}$ is obtained using Equation 5.5:

$$
M_{T}=\sqrt{\left(E_{T}+p_{t}\right)^{2}-\left(E_{T x}+p_{x}\right)^{2}-\left(E_{T y}+p_{y}\right)^{2}}
$$

where $p_{x}$ and $p_{y}$ are the components of the muon momentum in the $x$ and $y$ directions 
and $E_{T x}$ and $E_{T y}$ are the components of the $E_{T}$ in the $x$ and $y$ directions. If one assumes that the two leptons are massless this can be re-expressed as

$$
M_{T}=\sqrt{2 p_{t} E_{T}\left(1-\cos \phi_{12}\right)},
$$

where $\phi_{12}$ is the angular separation of the lepton and the missing energy. It can be seen that this equation is analogous to Equation 5.2, with transverse momentum substituted for total momentum. As the transverse momentum is always less than or equal to the total momentum this results in the transverse mass being being smaller than the invariant mass, resulting in a Jacobian peak, with a tail to low $m_{t}$. .

\subsection{Measurements using Electroweak Bosons}

There are two categories of studies being performed using electroweak bosons at the Tevatron. The first is to make measurements of the properties of the bosons themselves. The second is where knowledge of electroweak physics is used to probe other aspects of physics, such as detector resolution or non-perturbative QCD.

\subsubsection{Properties of Electroweak Bosons}

The first category of study is where better understanding of the properties of the boson themselves are sought. The most notable of these kind of measurements is that of the mass of the $\mathrm{W}$ boson. This can be used to constrain the range of masses allowed for the Higgs boson in the standard model, as described in Section 2.2.2.

The way that the mass of the $\mathrm{Z}$ boson was measured at the LEP experiments was to determine the position of the peak in the invariant mass distribution $[9]^{1}$. As

\footnotetext{
${ }^{1}$ Obviously the procedure was not quite this simple, with corrections needing to be applied for initial state radiation and other factors.
} 
noted above it is impossible to measure directly the invariant mass of the $\mathrm{W}$ and instead the transverse mass is used.

The procedure adopted for the measurement of the $\mathrm{W}$ mass in Run I was to compare the transverse mass distribution seen experimentally with a series of Monte Carlo samples generated with different $\mathrm{W}$ masses [43]. Although a discrete set of masses were generated a continuous distribution was obtained by interpolation. The mass of the $\mathrm{W}$ boson was determined to be the generated mass that provided the best agreement. It is also possible to do this with $p_{t}$ distribution of the lepton from W decay, which eliminates the need to understand the $E_{T}$ resolution. However, at present, this method is not as accurate as it is more sensitive to the modelling of various factors, including boson $p_{t}$.

Another measurement is that of the width of the $\mathrm{W}$ boson, which, with all the other electroweak precision measurements, can be used to over-constrain the Standard Model. The width can be determined using two methods. The first is directly by examining the transverse mass spectrum of $\mathrm{W}$ events in a similar way to the measurement of the $\mathrm{W}$ mass. The second method, used in the thesis, is to determine the width indirectly from the ratio of the $\mathrm{W}$ to $\mathrm{Z}$ production cross sections. This method is described in more detail in Section 5.3.

Similar measurements are also possible on the $\mathrm{Z}$ Boson, but the $\mathrm{Z}$ was studied to such precision at the LEP experiments [9] that it is impossible to improve on these results at the Tevatron.

\subsubsection{Electroweak Bosons as Calibration Tools}

A further use for events containing $\mathrm{W}$ and $\mathrm{Z}$ bosons is to help understand other physics processes. This utilises the fact that these processes have a clean signature, a relatively high production rate and have been extensively studied, so are well understood. There are two types of 'calibration': that where the electroweak processes 
are used to investigate detector resolution and efficiencies and that where these processes are used to improve understanding of the underlying physics of hadron-hadron colliders. Examples of how electroweak bosons are used to understand detector resolution are given in Section 8.1 and examples how they are used to determine particle identification and track reconstruction efficiencies are given in Section 8.2.

The second category is where the electroweak bosons are used to probe other physics, for instance non-perturbative QCD, those soft QCD processes to which no analytical solution is available. In particular the kinematic properties of the electroweak bosons and their decay products can be used to provide more information about the makeup of the proton.

The rapidity distribution of $\mathrm{W}$ and $\mathrm{Z}$ bosons can be used to probe the properties of the quarks inside the proton that the bosons are produced from. Specifically the differences in the rapidity distribution between the positively and negatively charged $\mathrm{W}$ bosons can be used to probe PDFs as mentioned in Chapter 7 . The $p_{t}$ distribution of the $\mathrm{Z}$ boson can be used to study the system recoiling off the $\mathrm{Z}$, which can be used to study the properties of QCD. A better model of boson $p_{t}$ would help a measurement of the mass of the $\mathrm{W}$, amongst other measurements.

Another way in which electroweak bosons can be utilised is to determine luminosity. This takes Equation 6.1 that is used to calculate the cross section, and instead of treating the cross section as an unknown, takes this value from the latest theoretical calculation and treats the luminosity as an unknown to be determined. In view of inherent problems in the present technique used to determine luminosity $\mathrm{W}$ and $\mathrm{Z}$ production rates may be used in future. The one area where this technique is already used to determine luminosity is in searches for states that have similar experimental signatures to electroweak bosons, for instance di-muon resonances. By normalising to the $\mathrm{Z} \rightarrow \mu^{+} \mu^{-}$peak not only does the luminosity cancel, but so do many of the efficiencies, reducing the sensitivity of an analysis to several factors. 


\subsection{Indirect Measurement of the W Boson Width}

The width of the $\mathrm{W}$ boson can be derived indirectly from the ratio of the $\mathrm{W} \rightarrow \mu \nu$ to $\mathrm{Z} \rightarrow \mu^{+} \mu^{-}$cross sections as follows:

$$
R \equiv \frac{\sigma(p \bar{p} \rightarrow \mathrm{W} \rightarrow \mu \nu)}{\sigma\left(p \bar{p} \rightarrow \mathrm{Z} \rightarrow \mu^{+} \mu^{-}\right)}=\frac{\sigma(p \bar{p} \rightarrow \mathrm{W}) \operatorname{Br}(\mathrm{W} \rightarrow \mu \nu)}{\sigma(p \bar{p} \rightarrow \mathrm{Z}) \operatorname{Br}\left(\mathrm{Z} \rightarrow \mu^{+} \mu^{-}\right)}
$$

where $\sigma(p \bar{p} \rightarrow \mathrm{W})\left(\equiv \sigma_{w}\right)$ and $\sigma(p \bar{p} \rightarrow \mathrm{Z})\left(\equiv \sigma_{z}\right)$ are the total production cross sections for the $\mathrm{W}$ and $\mathrm{Z}$ bosons respectively and $\operatorname{Br}()$ denotes the branching ratio to a particular process.

Re-expressing the branching ratio to leptons, in terms of partial widths the following expression can be obtained:

$$
R=\frac{\sigma_{W}}{\sigma_{Z}}\left(\frac{\Gamma_{Z}}{\Gamma_{Z \rightarrow \mu^{+} \mu^{-}}}\right)\left(\frac{\Gamma_{W \rightarrow \mu \nu}}{\Gamma_{W}}\right)
$$

The cross sections $\sigma_{W}$ and $\sigma_{Z}$ and the partial width of the $\mathrm{W}$ to a muon and a neutrino are all theoretically calculable. The ratio, $\frac{\Gamma_{Z}}{\Gamma_{Z \rightarrow \mu^{+} \mu^{-}}}$has been measured at LEP to high precision. Therefore if $R$ is measured the width of the $\mathrm{W}$ can be calculated. The remainder of this thesis describes the measurement of the width of the $\mathrm{W}$ boson using this technique. 


\section{Chapter 6}

\section{Measurement of $\mathrm{W} \rightarrow \mu \nu$ and}

\section{$\mathrm{Z} / \gamma^{*} \rightarrow \boldsymbol{\mu}^{+} \boldsymbol{\mu}^{-}$Cross Sections}

This chapter describes how the $\mathrm{W} \rightarrow \mu \nu$ and $\mathrm{Z} / \gamma^{*} \rightarrow \mu^{+} \mu^{-}$cross sections were evaluated. Both cross sections are determined using

$$
\sigma . \mathrm{Br}=\frac{N_{e v} C_{b k}}{\varepsilon\left(\int \mathcal{L} d t\right)}
$$

where $N_{e v}$ is the number of events passing the event selection, $C_{b k}$ corrects for background processes that pass the event selection, $\varepsilon$ is the efficiency of the event selection and $\int \mathcal{L} d t$ is the integrated luminosity of the data sample.

The $\mathrm{Z} / \gamma^{*} \rightarrow \mu^{+} \mu^{-}$cross section is measured as opposed to the $\mathrm{Z} \rightarrow \mu^{+} \mu^{-}$cross section as events from the photon and $\mathrm{Z}$ boson are indistinguishable. The obtention of the $\mathrm{Z} \rightarrow \mu^{+} \mu^{-}$cross section from this result is possible using a theoretical correction as described in Section 9.1. When the $\mathrm{Z} / \gamma^{*} \rightarrow \mu^{+} \mu^{-}$cross section is mentioned only events where the di-muon system has an invariant mass greater than $40 \mathrm{GeV}$ are considered. It is possible to measure the cross section for events with lower masses, but the background, in particular from b $\bar{b}$ events, becomes larger and more 
difficult to estimate. The mass cut is not chosen to be higher to minimise the effects of detector resolution.

The $\mathrm{Z} / \gamma^{*} \rightarrow \mu^{+} \mu^{-}$cross section is evaluated separately in three data taking periods due to changes in tracking efficiency and trigger configurations. These three periods are combined together for the final result. The $\mathrm{W} \rightarrow \mu \nu$ cross section is evaluated for two different trigger configurations which are then combined together for the final result.

This section gives a brief description of the analysis; it does not attempt to produce detailed justifications of event selections or accounts of why one method of estimating background is used as opposed to another. These technical details can be found in [35] and [36] for the $\mathrm{Z} / \gamma^{*} \rightarrow \mu^{+} \mu^{-}$analysis and [42] for the $\mathrm{W} \rightarrow \mu \nu$ analysis.

\subsection{Event Selections}

This section describes the requirements made on events to select reasonably pure samples of $\mathrm{Z} / \gamma^{*} \rightarrow \mu^{+} \mu^{-}$and $\mathrm{W} \rightarrow \mu \nu$ events. Both event selections start off by requiring a high $p_{t}$ muon to be detected. The $\mathrm{Z} / \gamma^{*} \rightarrow \mu^{+} \mu^{-}$event selection requires an additional high $p_{t}$ muon with opposite charge to the first. The $\mathrm{W} \rightarrow \mu \nu$ event selection requires evidence for a high $p_{t}$ neutrino in the form of $E_{T}$. Due to the larger backgrounds present for the $\mathrm{W} \rightarrow \mu \nu$ analysis, the selection requirements, for instance for muon identification, are tighter.

A muon is identified using the muon chambers and is required to be matched to a track in the central detector. All muons are required to be within the nominal acceptance of the muon detectors. This excludes the region where $\left|x_{A}\right|$ and $\left|y_{A}\right|$ are both less than $110 \mathrm{~cm}$, where the beampipe passes through to the central detector. The region where the muon system is compromised by the calorimeter supports, 
delimited by $4.25<\phi_{A}<5.15$ and $\left|\eta_{A}\right|<1.25$, is excluded.

Both the $\mathrm{Z} / \gamma^{*} \rightarrow \mu^{+} \mu^{-}$and $\mathrm{W} \rightarrow \mu \nu$ analyses require that the event fulfils one of a particular set of trigger conditions. These triggers combine various requirements from the different levels of the trigger system, as defined in Section 3.3. The di-muon trigger requires that two muons meet the tight scintillator requirement and that at least one muon is reconstructed at Level 2 as being of at least medium quality. The single muon trigger requires that at least one muon meets the loose wire requirement. A further requirement is that at least one muon is reconstructed by Level 2 as being of at least medium quality and with a minimum $p_{t}$ of $3 \mathrm{GeV}$. The final requirement of the single muon trigger is that at least one track, associated with a muon, is reconstructed by the Level 3 trigger with $p_{t}$ of at least $10 \mathrm{GeV}$. During certain periods the single muon trigger is switched off in the forward region $(|\eta|>1.5)$ to limit the amount of events recorded. This is known as the wide version of the single muon trigger, as opposed to the all version.

\subsection{1 $Z / \gamma^{*} \rightarrow \mu^{+} \mu^{-}$Event Selection}

The following additional event selection requirements are applied for the $\mathrm{Z} / \gamma^{*} \rightarrow \mu^{+} \mu^{-}$ analysis:

1. Both muons are required to have $p_{t}>15 \mathrm{GeV}$.

2. The invariant mass of the di-muon system is required to be greater than $40 \mathrm{GeV}$.

3. To reduce the background from b $\bar{b}$ events where both $b$ quarks decay semileptonically into muons, at least two out of the following four isolation criterion are required to pass:

(a) $\sum_{\text {tracks }, i} p_{t}^{i}<3.5 \mathrm{GeV}$, where $\sum_{\text {tracks }, i} p_{t}^{i}$ is the sum of the $p_{t}$ of tracks contained within a cone around the first muon direction with opening 
angle $R<0.5$, where $R^{2}=(\Delta \eta)^{2}+(\Delta \phi)^{2}$. The track associated with the selected muon, and tracks with a value of $z_{0}$ more than $2 \mathrm{~cm}$ from that of the candidate track are not considered in this sum.

(b) Same as (a) but for second muon.

(c) $\sum_{\text {cells }, i} E_{t}^{i}<2.5 \mathrm{GeV}$, where $\sum_{\text {cells }, i} E_{t}^{i}$ is the sum of the transverse energies of calorimeter cells in an annulus $0.1<R<0.4$ around the direction of the first muon.

(d) Same as (c) but for second muon.

4. To reduce the background from cosmic ray muons traversing the detector:

(a) $d c a<0.02 \mathrm{~cm}$ for tracks with hits in the SMT and $d c a<0.2 \mathrm{~cm}$ for muon tracks with no SMT hits.

(b) The angular separation, $\Delta \alpha$, between the two muons is required to be $\Delta \alpha>0.05$ radians, where $\Delta \alpha \equiv\left|\Delta \phi_{\mu \mu}+\Delta \theta_{\mu \mu}-2 \pi\right|$.

Before March 2003 the single muon trigger was heavily pre-scaled, so, to be selected, the event was required to fulfil the di-muon trigger requirements. This period is referred to as the first data taking period.

After this period then an event could be selected if it fulfilled either the di-muon or single muon trigger requirements. Only the wide version of the single muon trigger was used, to avoid the complications of evaluating the efficiencies for two versions of the single muon trigger.

There was a small amount of data collected after March 2003 with only the dimuon trigger. This is referred to as the second data taking period. The rest of the data is collected together as the third data taking period. The number of candidate events after this selection is 14,790 in an integrated luminosity of $147.7 \mathrm{pb}^{-1}$ [36]. 


\subsection{2 $\mathrm{W} \rightarrow \mu \nu$ Event Selection}

For the $\mathrm{W} \rightarrow \mu \nu$ analysis these additional selection criteria were applied

1. The muon is required to have $p_{t}>20 \mathrm{GeV}$.

2. The event is required to have $\mathbb{E}_{T}>20 \mathrm{GeV}$.

3. The transverse mass of the muon- $\mathbb{E}_{T}$ system, $m_{T}$, is required to be greater

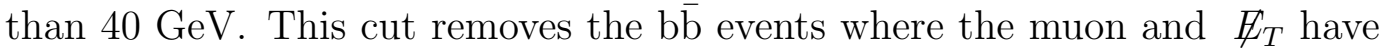
small angular separation, as the background fraction was found to be difficult to estimate for these events.

4. To remove background from $b \bar{b}$ events the following isolation cuts were applied. The isolation cuts are made upon the same variables as the $\mathrm{Z} / \gamma^{*} \rightarrow \mu^{+} \mu^{-}$ analysis, but are tighter due to the presence of larger $b \bar{b}$ backgrounds. The tightening of the cuts revealed a dependence on the instantaneous luminosity, $\mathcal{L}_{I}$, which is measured in units of $10^{30} \mathrm{~cm}^{-2} \mathrm{~s}^{-1}$. The average luminosity of the data sample was $\mathcal{L}=21.9$, with a standard deviation of 8.4. To remove the dependence of the efficiency on the luminosity, the cut is made to explicitly depend on instantaneous luminosity [42]:
(a) $\sum_{\text {cells }, i} E_{t}^{i}<\left(1.65+0.021 \mathcal{L}_{I}\right) \mathrm{GeV}$.
(b) $\sum_{\text {tracks, },} p_{t}^{i}<\left(1.1+0.014 \mathcal{L}_{I}\right) \mathrm{GeV}$

5. The central track matched to the muon is required to meet the following requirements:

(a) It must have at least one hit in the SMT associated with it.

(b) It must have a $\chi^{2}$ per degree of freedom of the track fit of less than 3.3 .

These requirements help minimise the contamination from mesons that decay in-flight. 
6. To further remove in-flight decays and cosmic rays, the $|d c a|$ of the track associated with the muon is required to be less than $110 \mu \mathrm{m}$.

7. To reduce the background from $\mathrm{Z} / \gamma^{*} \rightarrow \mu^{+} \mu^{-}$and cosmic rays, events are rejected if there is another muon in the event, passing the same quality requirements as the first muon. Events with a second central track with $p_{t}>20 \mathrm{GeV}$ and $\Delta \phi>2.1$ between the two tracks are also rejected. These are referred to as the muon veto and the track veto respectively.

8. The event is required to fulfil the single muon trigger requirements. The data is divided into two periods according to whether the single muon trigger is restricted to the wide region or not. These are referred to as the wide and all configurations respectively.

After applying these selection cuts 62,285 candidate events are obtained in a sample with an integrated luminosity of $96 \mathrm{pb}^{-1}$ [42].

A smaller data sample was used for the $\mathrm{W} \rightarrow \mu \nu$ analysis because the early data taking period, corresponding to the first data taking period of the $\mathrm{Z} / \gamma^{*} \rightarrow \mu^{+} \mu^{-}$ analysis was not used. This was because the trigger information was corrupted in such a way to make an efficiency measurement prohibitively difficult. A smaller percentage of the taken data was used due to the tighter requirements made on data quality.

\subsection{Measurement of Efficiencies}

The efficiency of both the $\mathrm{Z} / \gamma^{*} \rightarrow \mu^{+} \mu^{-}$and $\mathrm{W} \rightarrow \mu \nu$ event selection cuts were evaluated using the PMCS detector simulation as described in Section 4.3. This efficiency includes the probability for the muons in an event to lie within the geometrical and kinematical cuts made by the analyses and for the detector to identify them. The 
simulation also includes the probabilities for track and muon reconstruction and triggering efficiencies.

\subsubsection{Z $\mathrm{Z} / \gamma^{*} \rightarrow \mu^{+} \mu^{-}$Efficiencies}

Most of the efficiency is modelled by PMCS. However some quantities that the $\mathrm{Z} / \gamma^{*} \rightarrow \mu^{+} \mu^{-}$selection cuts upon, such as isolation variables are not simulated. Therefore the total efficiency for the process $\mathrm{Z} / \gamma^{*} \rightarrow \mu^{+} \mu^{-}$is given by the product of several efficiencies:

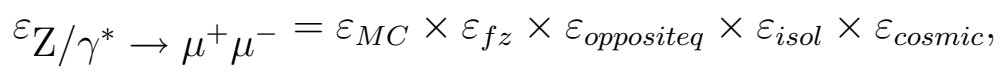

where $\varepsilon_{M C}$ is the efficiency evaluated with PMCS, including the acceptance, track reconstruction and muon identification efficiencies. The other efficiencies are the efficiency of the fast $\mathrm{z}$ requirement in the trigger $\left(\varepsilon_{f z}\right)$, the efficiency of requiring that the charges of the two muons are of the opposite sign $\left(\varepsilon_{\text {opposite }_{q}}\right)$, the efficiency of requiring that the event is isolated $\left(\varepsilon_{i s o l}\right)$ and the efficiency to pass the cosmic $\operatorname{cuts}\left(\varepsilon_{\text {cosmic }}\right)$.

A detailed description of the obtention of $\varepsilon_{M C}$ is given in Section 8.3.1. The rest of this section gives an overview of the methods used to obtain the other efficiencies. The only efficiencies treated differently in the different data taking periods are $\varepsilon_{M C}$ and $\varepsilon_{f z}$ due to the change in the trigger configurations and also to reflect the time dependence of the tracking efficiency [35].

$$
\text { - } \varepsilon_{f z}
$$

This is evaluated by looking at the fraction of $\mathrm{Z} \rightarrow e^{+} e^{-}$events, which are selected using a trigger with no fast-z requirement, that meet the fast $\mathrm{z}$ trigger requirement. In the latter two data taking periods, this requirement has been removed from the muon triggers, and this efficiency is trivially one. 


\begin{tabular}{|l|c|c|c|}
\hline Efficiency & Period 1 & Period 2 & Period 3 \\
\hline \hline$\varepsilon_{\text {isol }}$ & \multicolumn{3}{|c|}{$0.999 \pm 0.001$} \\
$\varepsilon_{\text {oppositeq }}$ & \multicolumn{3}{|c|}{$0.998 \pm 0.001$} \\
$\varepsilon_{\text {cosmic }}$ & \multicolumn{3}{|c|}{$0.988 \pm 0.006$} \\
\hline$\varepsilon_{f z}$ & $0.943 \pm 0.004$ & \multicolumn{2}{|c|}{$1.0 \pm 0.0$} \\
\hline$\varepsilon_{M C}$ & 0.239 & 0.268 & 0.322 \\
\hline \hline
\end{tabular}

Table 6.1: Summary of efficiencies in the $\mathrm{Z} / \gamma^{*} \rightarrow \mu^{+} \mu^{-}$analysis [35].

- $\varepsilon_{\text {opposite }_{q}}$ and $\varepsilon_{i s o l}$

Both of these efficiencies are obtained by examining the samples of events that pass all the event selections except the one we are interested in. The fraction of these events that are due to $\mathrm{Z} / \gamma^{*} \rightarrow \mu^{+} \mu^{-}$is then estimated to provide the efficiency.

- $\varepsilon_{\text {cosmic }}$

There are two cuts used to reject cosmic rays, the acolinearity and the dca cut. The acolinearity cut is evaluated using PMCS, but is treated separately. The efficiency of the dca cut is evaluated by plotting the dca of each of the muons against the dca of the other. In the case of cosmic rays the dca should be large and of approximately equal magnitude and of opposite sign. In the case of $\mathrm{Z} / \gamma^{*} \rightarrow \mu^{+} \mu^{-}$events the large $d c a$ arises from the track being badly reconstructed and so the $d c a$ of the two muons is less correlated. This method is used to estimate the fraction of events failing the $d$ ca cut are $\mathrm{Z} / \gamma^{*} \rightarrow \mu^{+} \mu^{-}$.

The efficiencies are summarised in Table 6.1. No uncertainties are given on $\varepsilon_{M C}$ as these are discussed in Section 8.3.1. 


\begin{tabular}{|l|c|c|}
\hline Efficiency & All & Wide \\
\hline \hline$\varepsilon_{M C}$ & 0.211 & 0.186 \\
\hline \hline
\end{tabular}

Table 6.2: Summary of efficiencies in the $\mathrm{W} \rightarrow \mu \nu$ analysis [44].

\subsection{2 $\mathrm{W} \rightarrow \mu \nu$ Efficiencies}

The total efficiency for the $\mathrm{W} \rightarrow \mu \nu$ analysis is determined using PMCs, denoted $\varepsilon_{M C}$. All of the selection variables in the analysis are modelled within PMCS. The variables included in this analysis that are not included in the $\mathrm{Z} / \gamma^{*} \rightarrow \mu^{+} \mu^{-}$analysis are added in two ways. The first is to include track quality cuts, such as those on the dca within the track reconstruction efficiency. The second, for the isolation efficiency, is to add a new variable into the simulation. It is possible to take both approaches

for the $\mathrm{W} \rightarrow \mu \nu$ analysis as each event only contains a single muon. Therefore no consideration needs to be made for correlations in the efficiencies between different muons in an event.

A detailed description of the technique used to evaluate $\varepsilon_{M C}$ can be found in [42]. The values are not exactly the same, due to several small changes in the analysis applied to obtain consistency with the $\mathrm{Z} / \gamma^{*} \rightarrow \mu^{+} \mu^{-}$analysis. One of these changes is that NLO instead of LO PDFs are used.

\subsection{Estimation of Background}

Wherever possible the backgrounds, especially QCD and instrumental backgrounds, are evaluated by studying data. The only exception are the electroweak backgrounds which produce signals that are very difficult to distinguish from candidate events. These arise from decay channels of electroweak bosons apart from the one that we are considering. For instance a $\mathrm{Z} / \gamma^{*} \rightarrow \mu^{+} \mu^{-}$event, where one of the muons remains 
undetected, is impossible to distinguish from a $\mathrm{W} \rightarrow \mu \nu$ event, therefore providing an irreducible background.

\subsubsection{Backgrounds to the $\mathrm{Z} / \gamma^{*} \rightarrow \mu^{+} \mu^{-}$Events}

There were five backgrounds that were considered to contribute to the candidate events:

- Cosmic rays traversing the detector are reconstructed as two muons originating from the beamspot. The fraction attributed to this background was denoted $f_{\text {cos }}$.

- $\mathrm{b} \overline{\mathrm{b}}$ events where both b-quarks decay semi-leptonically to muons. The fraction attributed to this background was denoted $f_{\mathrm{b}} \overline{\mathrm{b}}$. The background from $\mathrm{c} \overline{\mathrm{c}}$ events is expected to be negligible.

- $\mathrm{Z} / \gamma^{*} \rightarrow \tau^{+} \tau^{-}$events where both tau leptons decay to muons. The fraction attributed to this background was denoted $f_{\mathrm{Z} / \gamma^{*}} \rightarrow \tau^{+} \tau^{-}$.

- $\mathrm{W} \rightarrow \mu \nu$ events with an additional muon from an associated jet and di-boson events from WW, WZ and ZZ production, where two or more muons are produced. The fraction attributed to this background was denoted $f_{W}$.

These were combined to give the background correction

$$
C_{b k}=\frac{\left(1-f_{b \bar{b}}\right)\left(1-f_{\cos }\right)\left(1-f_{W}\right)}{\left(1+f_{Z \rightarrow \tau^{+} \tau^{-}}\right)}
$$

There are two ways of normalising a background fraction. One is with respect to the number of signal events; these backgrounds appear in the denominator of Equation 6.3. The other is with respect to the number of candidate events; these 
events appear in the numerator of Equation 6.3. For a more detailed explanation of this see Appendix A.

The fraction of events attributed to $\mathrm{b} \overline{\mathrm{b}}$ was estimated by looking at events passing all the event selection but failing the requirement that the two muons had opposite charges. Events with poorly reconstructed tracks were considered to be due to genuine $\mathrm{Z} / \gamma^{*} \rightarrow \mu^{+} \mu^{-}$events where one of the muons has a wrongly reconstructed charge. The other events were assumed to be due to $b \bar{b}$. This number of events was scaled up by the ratio of the $\mathrm{b} \overline{\mathrm{b}}$ production cross section for opposite-sign to like-sign di-muon pairs from mixing to produce an estimate of the b $\bar{b}$ background [45].

The fraction of events attributed to cosmic rays was estimated by inspecting the difference in the times that the muons arrive at the muon chambers.

The background from $\mathrm{Z} / \gamma^{*} \rightarrow \tau^{+} \tau^{-}$events was evaluated by passing a sample of $\mathrm{Z} / \gamma^{*} \rightarrow \tau^{+} \tau^{-}$events through the same Monte Carlo simulation used to evaluate the efficiency. The fraction attributed to $\mathrm{Z} / \gamma^{*} \rightarrow \tau^{+} \tau^{-}$is the ratio of the $\mathrm{Z} / \gamma^{*} \rightarrow \tau^{+} \tau^{-}$ to $\mathrm{Z} / \gamma^{*} \rightarrow \mu^{+} \mu^{-}$efficiencies.

The backgrounds from $\mathrm{W} \rightarrow \mu \nu+$ jet events and di-boson events were estimated by examining candidate events with three muons. Those events that had three isolated muons were attributed to being di-boson background. Those events that had one or more muons failing the isolation cuts were attributed to being due to $\mathrm{Z}+$ jet events. This fraction was scaled up by the ratio of the $\mathrm{W} \rightarrow \mu \nu$ to $\mathrm{Z} / \gamma^{*} \rightarrow \mu^{+} \mu^{-}$cross sections to estimate the number of $\mathrm{W} \rightarrow \mu \nu$ and jet events passing the $\mathrm{Z} / \gamma^{*} \rightarrow \mu^{+} \mu^{-}$event selection.

The different background fractions are summarised in Table 6.3. All were assumed to be unchanged between the different data taking periods. A more detailed description of the techniques used to estimate the size of the backgrounds can be found in [35] and [36]. 


\begin{tabular}{|l|c|}
\hline Background & Value \\
\hline \hline$f_{\cos }$ & $0.002 \pm 0.002$ \\
$f_{\mathrm{b} \overline{\mathrm{b}}}$ & $0.005 \pm 0.003$ \\
$f_{Z \rightarrow \tau^{+} \tau^{-}}$ & $0.005 \pm 0.001$ \\
$f_{W}$ & $0.002 \pm 0.001$ \\
\hline \hline
\end{tabular}

Table 6.3: Summary of backgrounds to the $\mathrm{Z} / \gamma^{*} \rightarrow \mu^{+} \mu^{-}$analysis.

\subsubsection{Backgrounds to $W \rightarrow \mu \nu$ Events}

There were two categories of backgrounds for the $\mathrm{W} \rightarrow \mu \nu$ analysis; those from other decays of electroweak bosons and those from other processes. The electroweak backgrounds, $\mathrm{Z} \rightarrow \mu^{+} \mu^{-}\left(f_{Z \rightarrow \mu^{+} \mu^{-}}\right), \mathrm{W} \rightarrow \tau \nu\left(f_{W \rightarrow \tau \nu}\right)$ and $\mathrm{Z} / \gamma^{*} \rightarrow \tau^{+} \tau^{-}\left(f_{Z \rightarrow \tau^{+} \tau^{-}}\right)$, were all evaluated using the same PMCS simulation used to evaluate the efficiency of the $\mathrm{W} \rightarrow \mu \nu$ event selection. Note that the $\mathrm{W} \rightarrow \tau \nu$ background has changed from that quoted in [42] due to the incorrect modelling of the polarisation of the $\tau$ leptons

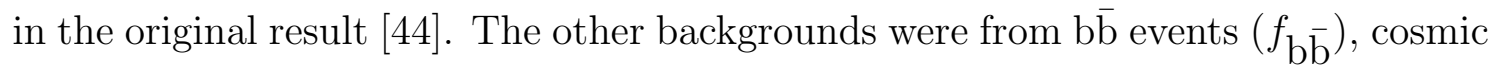
rays $\left(f_{c o s}\right)$ and in-flight decays $\left(f_{I F D}\right)$. The estimation of each of these backgrounds are described in more detail below.

All backgrounds are expressed as fractions of either the total numbers of $\mathrm{W} \rightarrow \mu \nu$ events, in the case of the electroweak backgrounds, or as fractions of the total number of candidate events, for all other background processes. Each of the backgrounds were combined to give the background correction

$$
C_{b k}=\frac{\left(1-f_{\mathrm{b} \overline{\mathrm{b}}}\right)\left(1-f_{\cos }\right)\left(1-f_{I F D}\right)}{\left(1+f_{\mathrm{Z} \rightarrow \mu^{+} \mu^{-}}\right)\left(1+f_{\mathrm{W} \rightarrow \tau \nu}\right)\left(1+f_{\mathrm{Z} / \gamma^{*} \rightarrow \tau^{+} \tau^{-}}\right.} .
$$

The b $\bar{b}$ background was evaluated using the matrix method. Because the efficiency is evaluated solely from data, c $\bar{c}$ events are also covered by this method. The matrix method uses a set of simultaneous equations to extract the number of back- 
ground and signal events in the candidate sample. Firstly all the event selection criteria, except the isolation cut which has a high rejection factor for the QCD background, are applied. This results in $N$ events of which $S$ are signal and $B$ are background:

$$
N=B+S
$$

Then the final event selection is applied resulting in $N_{2}$ events:

$$
N_{2}=\varepsilon_{b} B+\varepsilon_{s} S,
$$

where $\varepsilon_{b}$ and $\varepsilon_{s}$ are the efficiencies for the background and signal processes respectively. Knowing these efficiencies the equations can be solved to obtain an estimate of the amount of $b \bar{b}$ background in the candidate events. The difficult part is estimating $\varepsilon_{b}$, the efficiency of the isolation cuts for $\mathrm{b} \overline{\mathrm{b}}$ events.

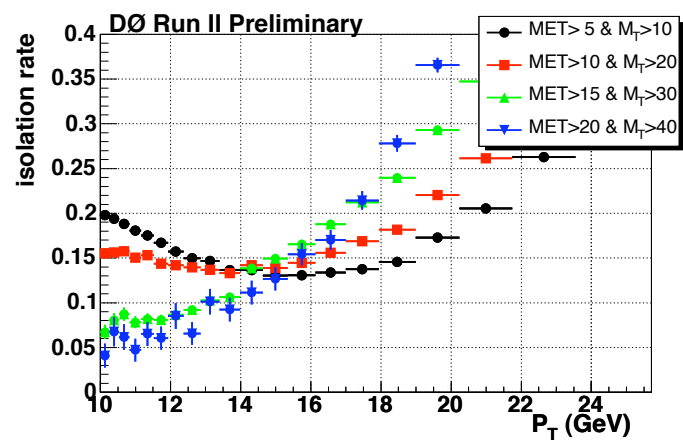

Figure 6.1: Isolation rate, in single muon events, as a function of the $p_{t}$ of the muon. The different bands represent different cuts on $\mathbb{E}_{T}$ and $m_{T}[42]$.

The efficiency for b $\bar{b}$ events is estimated using data in events with large amounts of $E_{T}$ and with a single muon with $p_{t}$ between ten and twelve GeV. Figure 6.1 shows the probability for single muons to pass the isolation cut as a function of $p_{t}$ of the muon. The different bands are for different $E_{T}$ and $m_{T}$ cuts. The efficiency 
for background events is taken from events with $E_{T}>20 \mathrm{GeV}$ and $m_{t}>40 \mathrm{GeV}$ in the low $p_{t}$ region, where the probability for a single muon to be isolated is 0.06 . In the high $p_{t}$ region this sample becomes "contaminated" with $\mathrm{W} \rightarrow \mu \nu$ events.

To extrapolate this efficiency into the high $p_{t}$ signal region, like sign di-muon events, which tend to be predominantly $\mathrm{b} \overline{\mathrm{b}}$ events, are used. The probability for the muons in these events to be isolated shows no sign of increase with $p_{t}$. The different kinematics of these events means that we cannot use them to get an absolute value, but the general behaviour is assumed to be the same.

As it is impossible to extract a definite value of the background efficiency from the signal region the value $0.03 \pm 0.03$ is taken. This is done by halving the upper bound and quoting an uncertainty spreading from zero to the upper bound.

Kaons and pions can decay to muons before showering in the calorimeter. This can produce an isolated muon in the muon chambers in association with a track in the central detector, faking a $\mathrm{W} \rightarrow \mu \nu$ event. Trying to fit a single track from the combination of the meson and the muon tracks tends to produce a larger $\chi^{2}$ in the track fit and a poorer $d c a$ and $p_{t}$ resolution. Combining this poor $p_{t}$ resolution with the fact that the muon produced in the decay tends to have lower $p_{t}$, results in a discrepancy between the $p_{t}$ as measured by the muon system and that as measured by the central tracker. Cosmic rays can pass the $\mathrm{W} \rightarrow \mu \nu$ event selection if they are reconstructed in both the muon system and the tracker. As they are not produced in the interaction point, they tend to have large values of $d c a$.

It is the $d c a$ distribution that is used to discriminate the signal events from these backgrounds. The procedure adopted is to create template $d c a$ distributions for signal and these backgrounds. The $d c a$ distribution in the candidate events is then fitted with a sum of these templates to estimate the fraction of candidates that are due to in-flight decay (IFD) and cosmic backgrounds.

The template for the signal is taken from $\mathrm{Z} \rightarrow \mu^{+} \mu^{-}$events in data which are 


\begin{tabular}{|l|c|c|}
\hline Background & All & Wide \\
\hline \hline$f_{\mathrm{Z} \rightarrow \mu^{+} \mu^{-}}$ & $0.043 \pm 0.002$ & $0.041 \pm 0.002$ \\
$f_{\mathrm{W} \rightarrow \tau \nu}$ & $0.031 \pm 0.001$ & $0.031 \pm 0.001$ \\
$f_{\mathrm{Z} / \gamma^{*} \rightarrow \tau^{+} \tau^{-}}$ & $0.0018 \pm 0.0001$ & $0.0018 \pm 0.0001$ \\
$f_{\mathrm{b} \overline{\mathrm{b}}}$ & $0.007 \pm 0.007$ & $0.008 \pm 0.008$ \\
\hline$f_{I F D}$ & \multicolumn{2}{|c|}{$0.0022 \pm 0.0012$} \\
$f_{\text {cos }}$ & 0 \\
\hline \hline
\end{tabular}

Table 6.4: Summary of backgrounds to the $\mathrm{W} \rightarrow \mu \nu$ analysis [42].

assumed to have no background from cosmic ray events ${ }^{1}$. The template distributions for the IFD and cosmic ray backgrounds are taken from samples enhanced in the relevant background. The $d c a$ distribution of the candidate events is fitted using the three templates to obtain the fraction of events attributed to signal and backgrounds.

Following this procedure the background from cosmic rays is found to be negligible and that from in-flight decays found to be $(0.0022 \pm 0.0012)$. The uncertainty on this number is a systematic error obtained by varying the sample on which the template for the IFD background is fitted from.

The different background fractions are summarised in Table 6.4. A more detailed description of the techniques used to estimate the size of the backgrounds can be found in [42].

\subsection{Integrated Luminosity}

This section gives an overview of the methods used to obtain the integrated luminosity of a data sample at DØ. A more detailed description can be found in [46]. The

\footnotetext{
${ }^{1}$ The small cosmic ray background in the $\mathrm{Z} / \gamma^{*} \rightarrow \mu^{+} \mu^{-}$analysis is eliminated by applying the tighter track quality requirements of the $\mathrm{W} \rightarrow \mu \nu$ analysis.
} 
integrated luminosity is obtained using

$$
\int \mathcal{L} d t=\frac{N_{\mathrm{p} \overline{\mathrm{p}}}}{\varepsilon_{L M} \sigma_{\mathrm{p} \overline{\mathrm{p}}}},
$$

where $N_{\mathrm{p} \overline{\mathrm{p}}}$ is the number of inelastic p $\overline{\mathrm{p}}$ collisions observed by the luminosity monitor as described in Section 3.2.5, $\varepsilon_{L M}$ is the efficiency for the luminosity monitor to observe inelastic collisions [46] and $\sigma_{\mathrm{p}} \overline{\mathrm{p}}$ is the total inelastic $\mathrm{p} \overline{\mathrm{p}}$ cross section, which has been measured at a centre of mass energy, $\sqrt{s}=1.8 \mathrm{TeV}$ by the CDF [47] and E811 [48] experiments. These results are combined and extrapolated to $\sqrt{s}=$ $1.96 \mathrm{TeV}$ to produce a result of $\sigma_{\mathrm{p} \overline{\mathrm{p}}}=60.7 \pm 2.4 \mathrm{mb}$ [49]. Combining the uncertainties on $\varepsilon_{L M}$ and $\sigma_{\mathrm{p}} \overline{\mathrm{p}}$ results in an overall uncertainty of $6.5 \%$ on the integrated luminosity.

\subsection{Summary of Results}

The cross sections are obtained using Equation 6.1. The four numbers required to produce the cross sections are summarised in Table 6.5. The cross sections from the three data taking periods, for the $\mathrm{Z} / \gamma^{*} \rightarrow \mu^{+} \mu^{-}$cross section, and the two trigger configurations, for the $\mathrm{W} \rightarrow \mu \nu$ cross section, are combined to produce a single value for each cross section. These combinations are weighted by the uncorrelated errors on each of the contributing cross sections. All statistical uncertainties, such as that on the number of candidate events, are uncorrelated. The systematic uncertainties on the efficiencies and the backgrounds are discussed in more detail in Chapters 8 and 9 . 


\begin{tabular}{|c|c|c|c|c|c|}
\hline Process & $N_{e v}$ & $C_{b k}$ & $\varepsilon$ & $\int \mathcal{L} d t\left(p b^{-1}\right)$ & Cross Section $(p b)$ \\
\hline $\mathrm{Z} / \gamma^{*} \rightarrow \mu^{+} \mu^{-}($Period 1$)$ & 2650 & 0.986 & 0.222 & 36.8 & 320 \\
\hline $\mathrm{Z} / \gamma^{*} \rightarrow \mu^{+} \mu^{-}($Period 2$)$ & 146 & 0.986 & 0.264 & 1.5 & 365 \\
\hline $\mathrm{Z} / \gamma^{*} \rightarrow \mu^{+} \mu^{-}($Period 3$)$ & 11556 & 0.986 & 0.317 & 109.4 & 329 \\
\hline $\mathrm{Z} / \gamma^{*} \rightarrow \mu^{+} \mu^{-}($Total $)$ & & & & & 328 \\
\hline $\mathrm{W} \rightarrow \mu \nu($ All $)$ & 29159 & 0.918 & 0.211 & 41.9 & 3030 \\
\hline $\mathrm{W} \rightarrow \mu \nu$ (Wide) & 33126 & 0.926 & 0.186 & 53.7 & 3070 \\
\hline $\mathrm{W} \rightarrow \mu \nu($ Total $)$ & & & & & 3050 \\
\hline
\end{tabular}

Table 6.5: Summary of numbers used to produce the cross sections. 


\section{Chapter 7}

\section{Parton Distribution Functions}

This chapter describes work toward a systematic determination of the uncertainty due to the choice of Parton Distribution Function (PDF). It starts with a general overview of the theory behind PDFs in Section 7.1, before providing a more detailed description of the PDF sets used in this analysis in Section 7.2. A brief discussion of uncertainties on PDF sets is given in Section 7.3 before the chapter is concluded in Section 7.4 with a description of techniques used to translate uncertainties on PDF sets into uncertainties on observables.

\subsection{Theoretical Background}

Although the Tevatron is used to collide protons and antiprotons, the actual interactions involve the constituent partons. PDFs describe the probability of finding a parton with a fraction, $x$, of the total momentum inside a hadron at an energy squared scale, $Q^{2}$.

PDFs rely on the ability to separate the high energy hard scatter from the low energy interactions within the hadron, known as factorisation. This involves 
introducing a cutoff in the model, the factorisation scale $\left(\mu_{F}\right)$. In simple terms, a gluon emitted with $p_{t}$ less than the factorisation scale is treated as part of the hadron, whilst one emitted with $p_{t}$ greater than the factorisation scale is treated as part of the hard scatter. In practice $\mu_{F}^{2}$ is chosen to be a value of the order of the an energy scale squared related to the hard scatter, $Q^{2}$.

Given the ability to separate the hard scatter from all the soft underlying interactions within the colliding hadrons, the problem reduces to determining the probability of resolving a parton of a given type and momentum from the proton. Although these probabilities vary with $\mu_{F}$, the way they vary is understood and can be compared between different values of $\mu_{F}$ using the DGLAP equation [50]. Therefore a single set of PDFs can be obtained from all data and used to make universal predictions.

Because the QCD interactions within hadrons are at low energies it is impossible to calculate them perturbatively. Instead of trying to derive PDFs from first principles the approach taken is to produce a parameterised dependence on $x$. The values of the parameterisation are obtained by fitting to a wide variety of experimental results. In order to obtain as reliable a fit as possible, data with a wide range of $x$ and $Q^{2}$ values are taken, as shown in Figure 7.1.

It can be seen that the data used here was predominantly from the ep collider, HERA, and fixed target deep inelastic scattering (DIS) and Drell Yan (DY) experiments, although some results from the Tevatron, such as the $\mathrm{W}$ asymmetry (as discussed in Chapter 5) and the high transverse momentum jet cross section, are also used. The different types of data also probe different aspects of the PDFs with, for instance, the $\mathrm{W}$ asymmetry probing the asymmetry between up and down quarks and the jet cross section probing the gluon distribution.

Because the data has some experimental uncertainty there is also an uncertainty associated with the parameters used. Until recently no systematic method was avail- 


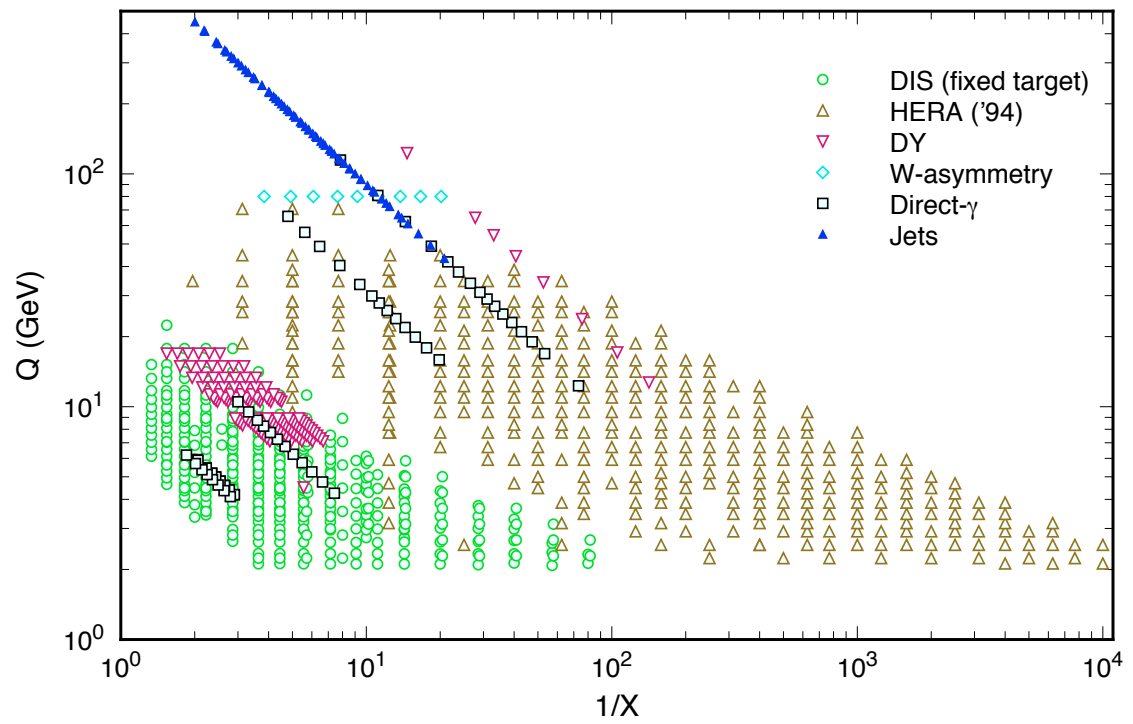

Figure 7.1: Distribution of $\frac{1}{x}$ and $Q$ values used in global fit used to produce the CTEQ5 PDFs [51].

able to assess quantitatively the propagation of this uncertainty into experimental observables, such as cross sections. The CTEQ [52] and MRST [53] collaborations have recently developed techniques to allow a quantitative assessment of these uncertainties. In order to better illustrate the evaluation of the uncertainties using these methods, a more detailed description will be given of the CTEQ6 PDF sets and the method used to evaluate the uncertainty due to the choice of PDF.

\subsection{The CTEQ6 PDF Sets}

The CTEQ6 PDF sets are chosen for more detailed description as they are utilised by this analysis. This section provides a brief introduction; an exhaustive description can be found in $[52,54]$. The same PDFs can be used for antiprotons by swapping partons for their antipartons. Other PDF sets are available for particles such as pions. 
CTEQ6 includes the following partons; the $u, d, s$ quarks, their antiquarks and the gluon. The $s$ and $\bar{s}$ distributions are assumed to be the same and equal to

$$
s(x)=\bar{s}(x)=0.2(\bar{u}(x)+\bar{d}(x)) .
$$

The remaining distributions are arranged into the valence quark distributions and the sea parton distributions as follows.

- The valence up quark distribution: $u_{v}(x) \equiv u(x)-\bar{u}(x)$

- The valence down quark distribution: $d_{v}(x) \equiv d(x)-\bar{d}(x)$

- The gluon distribution: $g(x)$

- The antiquark sea distribution: $\bar{u}(x)+\bar{d}(x)$

- The light antiquark ratio: $(\overline{u(x)} / \overline{d(x)})$

All distributions are parameterised as a function of $x$ and fitted at a factorisation scale of $\mu_{F}=1.3 \mathrm{GeV}$. As long as a degree of flexibility is allowed the exact form of the parameterisation is not important. The first four use the form

$$
x f(x)=A_{0} x^{A_{1}}(1-x)^{A_{2}} e^{A_{3} x}\left(1+e^{A_{4}} x\right)^{A_{5}},
$$

where $A_{0}$ to $A_{5}$ are fit parameters. These parameters are different for all four distributions, resulting in 24 different variables. The last distribution is parameterised

$$
\frac{\bar{d}\left(x ; \mu_{F}\right)}{\bar{u}\left(x ; \mu_{F}\right)}=A_{0} x^{A_{1}}(1-x)^{A_{2}}+\left(1+A_{3} x\right)(1-x)^{A_{4}},
$$

where $A_{0}$ to $A_{4}$ are five other tunable parameters. This leaves a total of 29 free parameters. When comparing this model to the data the fit did not converge. This 
problem was overcome by fixing some of the parameters ${ }^{1}$ leaving a total of 20 free parameters. The values of the 20 free parameters are obtained by minimising the $\chi^{2}$ of the global fit between data and the model.

\subsection{Uncertainties on PDFs}

The global fit has a degree of uncertainty attached to it due to experimental uncertainties on the input data. This results in each of the fitted parameters having an attached uncertainty. The parameters described in Section 7.2 are correlated with each other making any assessment of the uncertainty on any given parameter complicated. Therefore when dealing with uncertainties 20 new parameters are used, which can be denoted $B_{i}$, where $i$ runs from 1 to 20 . These parameters are combinations of the original parameters, chosen to be orthogonal to each other.

The procedure adopted in the CTEQ6 PDF sets is to create 40 PDF sets in addition to the central one. Each parameter has two of these additional sets associated with it, one where the parameter has been increased in such a way as to decrease the quality of the global fit by one error unit (the up error sets) and one where the parameter has been decreased in such a way as to decrease the quality of the global fit by one error unit (the down error sets).

The vague term error unit is deliberately employed because the $\chi^{2}$ per degree of freedom is greater than one. Instead of choosing to take the error sets as being where the global $\chi^{2}$ is increased by one, it is chosen to be increased by 100 . This number is arbitrary with, for instance the MRST collaboration taking this number to be 50 .

\footnotetext{
${ }^{1} \mathrm{As}$ the parameterisation is arbitrary this is a perfectly legitimate technique
} 


\subsection{Uncertainties on Observables}

This section describes techniques that use the different CTEQ error PDFs to produce an uncertainty on an observable, such as an acceptance or a cross section. To assess an uncertainty on an observable $(\Delta X)$ due to choice of PDF, the observable $(X)$ is evaluated with the central PDF set $\left(S_{0}\right)$ and the 40 error sets $\left(S_{i}\right)$. The observables chosen to illustrate these techniques are calculations of the $\mathrm{Z} \rightarrow \mu^{+} \mu^{-}$ and $\mathrm{Z} / \gamma^{*} \rightarrow \mu^{+} \mu^{-}$cross sections using the MC@NLO generator [29]. The ratio of these cross sections $\left(R_{\sigma} \equiv \mathrm{Z} \rightarrow \mu^{+} \mu^{-} / \mathrm{Z} / \gamma^{*} \rightarrow \mu^{+} \mu^{-}\right)$is also used as this is more sensitive to the prescription used to evaluate PDF uncertainty.

The $\mathrm{Z} \rightarrow \mu^{+} \mu^{-}$cross section was calculated using the central PDF set and the 40 error sets. The central value $\sigma=236.4 \pm 0.1 \mathrm{pb}$ agrees well with the full NLO calculation by Van Neervan et al, $\sigma=236.2 \mathrm{pb}[55,56]$. Figure 7.2(a) shows, for each of the 40 error sets, the fractional difference to the central value.

The original prescription, devised by the CTEQ collaboration [52], to convert these values into an uncertainty, $\Delta X$, is

$$
\Delta X=\left(\frac{1}{2} \sum_{i=1}^{20}\left[X\left(S_{i}^{+}\right)-X\left(S_{i}^{-}\right)\right]^{2}\right)^{1 / 2}
$$

This assumes that the value of the observable calculated using the 'up' and 'down' error sets is displaced by equal amounts in opposite directions from the central value. Examining Figure 7.2 this appears to be a reasonable assumption for the lower error PDFs. When looking at the higher error PDFs the displacement of the observable from the central value becomes asymmetric and there are even cases where the displacement of the observable for the 'up' and 'down' sets is in the same direction..

The CTEQ collaboration reworked the error sets to try and reduce any asymmetries, but acknowledged that the effect was real. Therefore the old prescription to evaluate the PDF uncertainty could no longer be regarded as valid and a new 

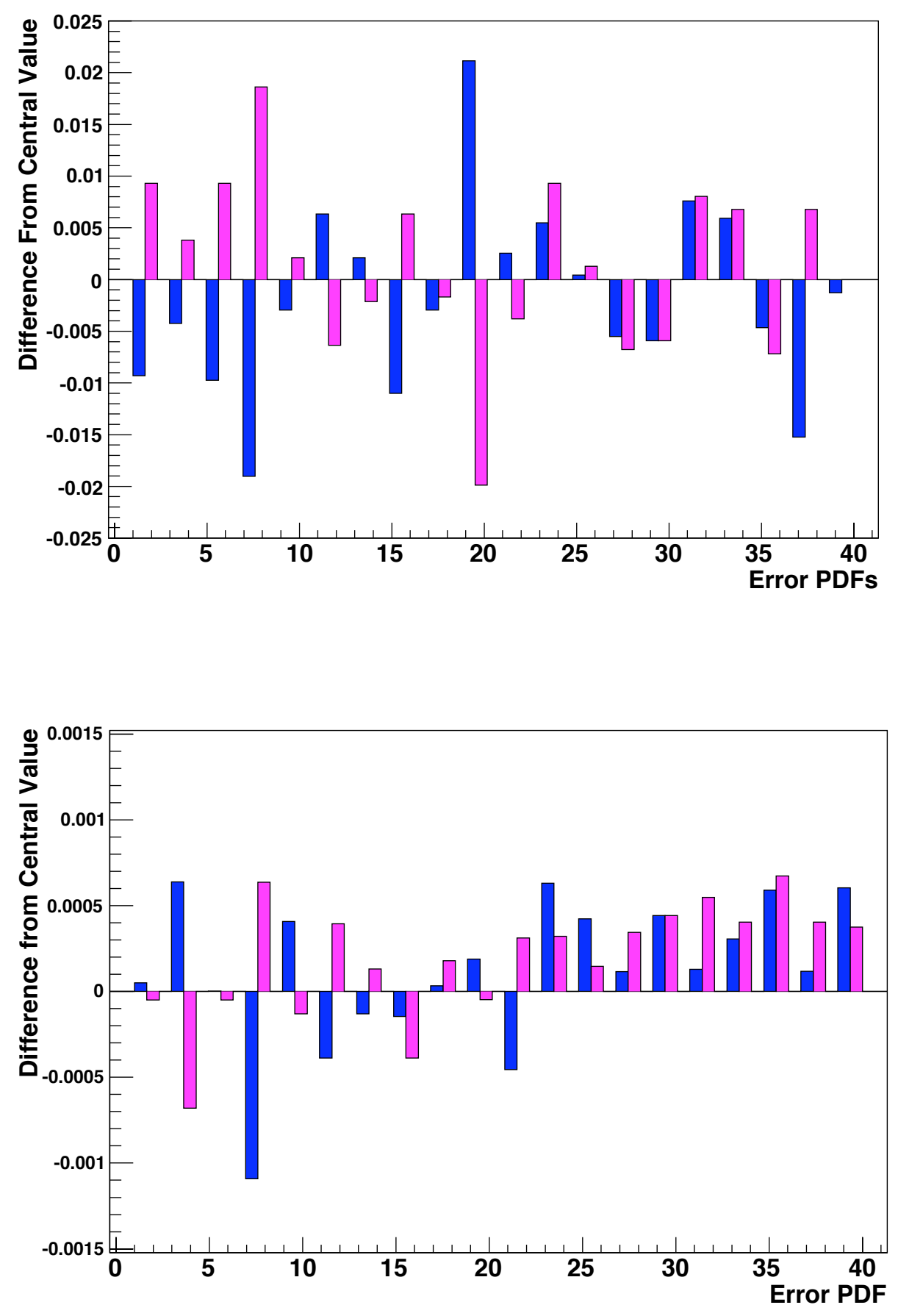

Figure 7.2: Fractional difference between each of the 40 error sets and the central PDF for the $\mathrm{Z} \rightarrow \mu^{+} \mu^{-}$cross section (top plot) and $R_{\sigma}$. The 'up' error sets are shaded blue and the 'down' error sets are shaded magenta. 
prescription was formed [54]. This new prescription assumes that, in general, the uncertainty on an observable due to choice of PDF is asymmetric. Therefore the error sets in which the observable is larger than the central value $\left(X_{+}\right)$are treated separately from those in which the error sets in which the observable is smaller than the central value $\left(X_{-}\right)$. Each of these cases are summed separately to produce the asymmetric errors $\left(\Delta X_{ \pm}\right)$.

This is described as follows:

$$
\Delta X_{ \pm}=\left(\sum_{i=1}^{20}\left[X^{ \pm}\left(S_{j}\right)-X\left(S_{0}\right)\right]^{2}\right)^{1 / 2}
$$

Note that the subscript on the $X$ denotes that it is unimportant whether we are treating the up or down error set. The important factor is whether the observable with a given error set is larger, or smaller, than the central value.

This prescription retains one implicit assumption, that the displacement of the up and the down error sets are in opposite directions. As can be seen from inspection of Figure 7.2 this is not always the case. It can be seen that there are many pairs of error sets where the up and down sets are displaced in the same direction. As the up and the down sets are not independent it would not be correct to add them both to the same sum. Therefore a new prescription has to be constructed to take this into account.

The standard $D \varnothing$ prescription is to only add the case with the larger displacement from the central value. The TeVEWWG prescription ${ }^{2}$ is, if the observable is displaced in the same direction using both the up and down sets, the average of the two is added to the appropriate sum. In practice the difference between the two approaches is minimal. Table 7.1 summarises the PDF uncertainty on the $\mathrm{Z} \rightarrow \mu^{+} \mu^{-}$cross section and its ratio to the $\mathrm{Z} / \gamma^{*} \rightarrow \mu^{+} \mu^{-}$cross section with the

\footnotetext{
${ }^{2}$ The TevEWWG is the Tevatron Electroweak Working Group, designed to co-ordinate measurements of electroweak physics between the $\mathrm{D} \emptyset$ and CDF experiments.
} 
different prescriptions.

\begin{tabular}{|l|c|c|}
\hline Prescription & $\sigma_{\mathrm{Z} / \gamma^{*} \rightarrow \mu^{+} \mu^{-}}$ & $R_{\sigma}$ \\
\hline 'old' CTEQ & $\pm 3.5 \%$ & $\pm 0.1 \%$ \\
TevEWWG & $+3.5 \% /-3.9 \%$ & $\pm 0.2 \%$ \\
D & $+3.6 \% /-3.9 \%$ & $\pm 0.2 \%$ \\
\hline
\end{tabular}

Table 7.1: PDF Uncertainty on the $\mathrm{Z} / \gamma^{*} \rightarrow \mu^{+} \mu^{-}$cross section and $R_{\sigma}$ using the prescriptions described above. 


\section{Chapter 8}

\section{Studies for the PMCS Simulation}

This chapter consists of a collection of studies performed for the PMCS simulation. Additional work was performed by the author on the simulation but, being maintenance or technical work and insusceptible to description, is not included. This other work included the writing of documentation $[57,58]$.

This chapter begins in Section 8.1 by describing work done to develop the tracking resolution parameterisation and tuning the parameters to optimise the agreement with data. It proceeds to describe the modelling of the efficiencies for tracking and muon identification and triggering in Section 8.2, before concluding in Section 8.3 with a description of some applications of the simulation, in particular in the calculation of the efficiency of the $\mathrm{Z} / \gamma^{*} \rightarrow \mu^{+} \mu^{-}$event selection.

\subsection{Modelling Track Resolution}

The modelling of the $p_{t}$ resolution of the tracker is based upon three variable parameters, as described in Section 4.3.1. 


$$
\begin{gathered}
\frac{\sigma_{1 / p_{t}}}{1 / p_{t}}=\sqrt{A^{2} \frac{p_{t}^{2}}{L^{4}}+\frac{B^{2}}{L \sin (\theta)}} \\
p_{t}(\text { smear })=C p_{t}^{\prime}
\end{gathered}
$$

The first stage involves smearing the $p_{t}$ according to a Gaussian with a width determined by Equation 8.1. One of the parameters, $A$, models the sensitivity of the resolution to measurement error on the sagitta which dominates the resolution at high $p_{t}$. Another parameter, $B$, models the sensitivity of the resolution to multiple scattering of the particles as they pass through the tracker, which dominates the resolution at low $p_{t}$. The smeared $p_{t}$ is then multiplied by a scale factor, $C$, to account for an imperfect description of the magnetic field in the reconstruction process and other factors.

The parameters were not obtained through any a priori reasoning, but by optimising agreement between the simulation and data. This process involves the use of two di-muon resonances, $\mathrm{Z} \rightarrow \mu^{+} \mu^{-}$and $\mathrm{J} / \Psi \rightarrow \mu^{+} \mu^{-}$. The $\mathrm{Z} \rightarrow \mu^{+} \mu^{-}$peak provides a sample of muons with high $p_{t}$, whilst the muons from the $\mathrm{J} / \Psi \rightarrow \mu^{+} \mu^{-}$ peak have, on average, lower $p_{t}$.

This section gives a detailed description of the tuning process. The first step involves the tuning of the value of $A$ and $C$ using high $p_{t}$ muons from $\mathrm{Z} / \gamma^{*} \rightarrow \mu^{+} \mu^{-}$

events. The second step involves the tuning of parameter, $B$, using the lower $p_{t}$ muons from $\mathrm{J} / \Psi \rightarrow \mu^{+} \mu^{-}$events. The process was iterative, so preceding the steps described below all three parameters had been roughly tuned.

\subsubsection{Studies using using $Z \rightarrow \mu^{+} \mu^{-}$Events}

A sample of $\mathrm{Z} / \gamma^{*} \rightarrow \mu^{+} \mu^{-}$events was selected using the event selection described in Section 6.1.1. These were used to tune the parameters relating to the measurement 
error, $A$, and the $p_{t}$ scale, $C$. Samples of $\mathrm{Z} / \gamma^{*} \rightarrow \mu^{+} \mu^{-}$events were generated using PMCS with varying values of $A$ and $C$, and those events that passed the event selection retained. To be consistent with other PMCs tunes a mass window cut of 65 to $105 \mathrm{GeV}$ was applied around the $\mathrm{Z} \rightarrow \mu^{+} \mu^{-}$peak [33].

The comparison was performed by normalising the total number of events in the PMCS samples to the total number of events in the data. The distribution of events, as a function of mass, was obtained for both the data and the PMCs samples. The two distributions were compared to produce a $\chi^{2}$ value, defined as follows:

$$
\chi^{2} \equiv \sum_{i} \frac{\left(N_{i}^{\text {data }}-N_{i}^{M C}\right)^{2}}{\left(\sigma_{i}^{\text {data }}\right)^{2}+\left(\sigma_{i}^{M C}\right)^{2}},
$$

where the sum is performed of the difference in the number of entries between the data, $N_{i}^{d a t a}$, and the normalised Monte Carlo simulation, $N_{i}^{M C}$, over the different mass bins, $i$. In each mass bin the difference is divided by the statistical uncertainty, which is obtained from the statistical uncertainty on the data, $\sigma_{i}^{\text {data }}$, and that on the Monte Carlo, $\sigma_{i}^{M C}$. The optimum parameterisation was the one that produces the best agreement, i.e. the lowest $\chi^{2}$ value.

The track resolution is sensitive to many factors. Tighter requirements made on the quality of the tracks improve the resolution. This implies that the same tune cannot be used for both the $\mathrm{Z} / \gamma^{*} \rightarrow \mu^{+} \mu^{-}$and $\mathrm{W} \rightarrow \mu \nu$ analyses due to the tighter track quality requirements made in the $\mathrm{W} \rightarrow \mu \nu$ analysis. The approach adopted was to produce separate tunes for the two analyses.

The tune was first performed for the $\mathrm{Z} / \gamma^{*} \rightarrow \mu^{+} \mu^{-}$analysis using all selected events. The variation of $\chi^{2}$ versus $A$ is shown in Figure 8.1(a). The optimum value of $A$ was determined, by fitting a third order polynomial to the data in Figure 8.1(a), to be 


$$
A=0.00292 \pm 0.00010 \mathrm{GeV}^{-1} \text {. }
$$

The variation of $\chi^{2}$ versus $C$ is shown in Figure 8.1(b).

The optimum value of $C$ was determined, by fitting a third order polynomial to the data in Figure 8.1(b), to be

$$
C=0.992 \pm 0.003
$$

The fit has a $\chi^{2}$ per degree of freedom of two at the optimum point. The fact that the optimum value of $\chi^{2}$ is greater than one reflects two things; no systematic uncertainty, such as that caused by the shape of backgrounds, is included in the $\chi^{2}$ calculation and the model is too simple to provide a perfect description. The systematic uncertainty from the fit was estimated by allowing the $\chi^{2}$ of the fit to increase by two and seeing how the value of the parameter varies. Other sources of systematic uncertainty, such as the shape of backgrounds, the values of other parameters, changing the function used to fit the variation of the $\chi^{2}$ and varying the range of the mass window were investigated and found to be negligible.

The tuning was repeated for the $\mathrm{W} \rightarrow \mu \nu$ analysis. This was done by applying the track quality cuts used by the $\mathrm{W} \rightarrow \mu \nu$ analysis, as described in Section 6.1.2, on the sample of $\mathrm{Z} / \gamma^{*} \rightarrow \mu^{+} \mu^{-}$events used above.

The variation of $\chi^{2}$ versus $A$ is shown in Figure 8.2(a). The optimum value of $A$ was determined, by fitting a third order polynomial to the data in Figure 8.2(a), to be

$$
A=0.00235 \pm 0.00010 \mathrm{GeV}^{-1}
$$

The variation of $\chi^{2}$ versus $C$ is shown in Figure 8.2(b). The optimum value of $C$ 

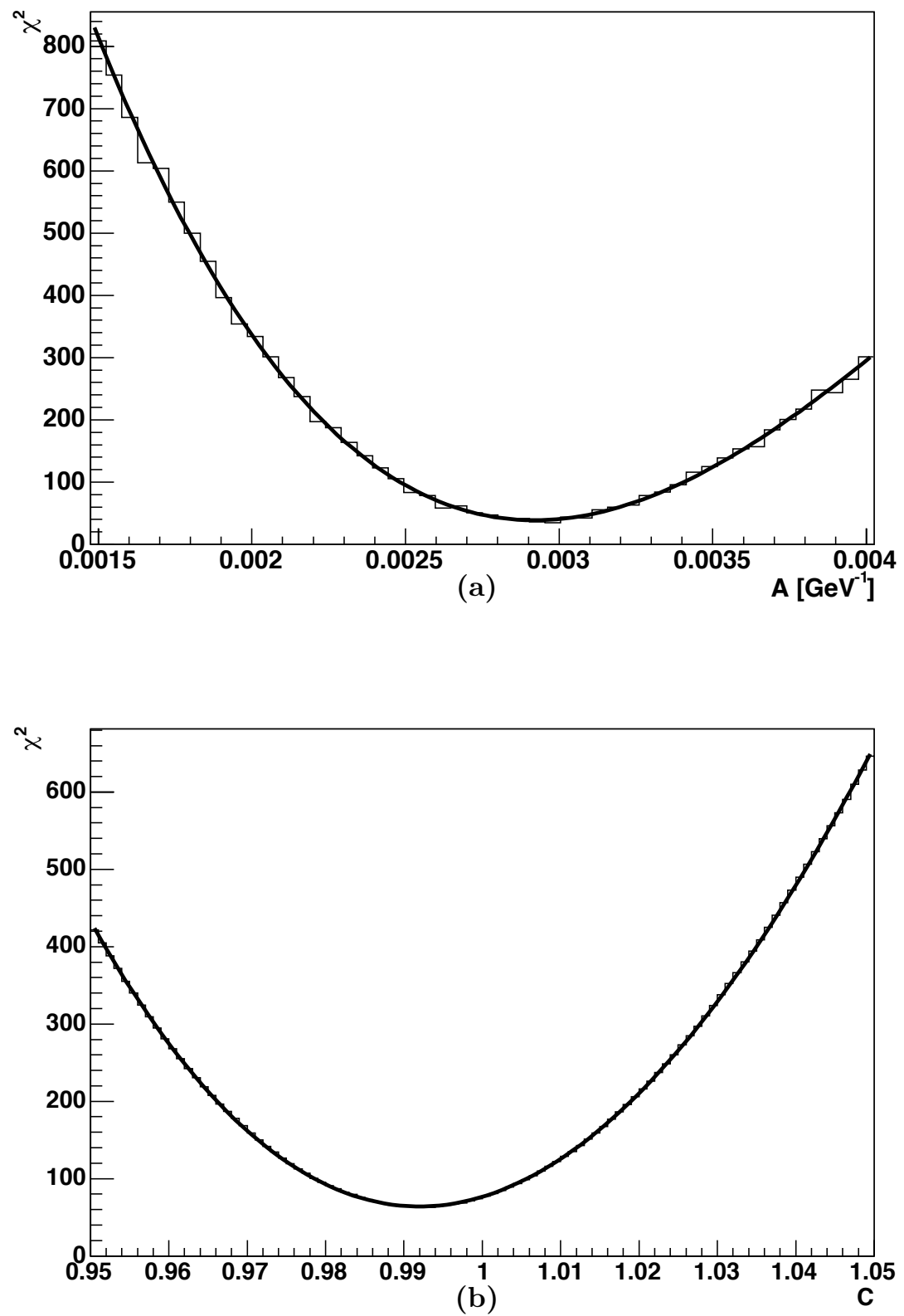

Figure 8.1: $\chi^{2}$ comparison of $\mathrm{Z} \rightarrow \mu^{+} \mu^{-}$peak between data and PMCS with varying values of $A$ in the top plot and $C$ in the bottom plot . 
was determined, by fitting a third order polynomial to the data in Figure 8.2(b), to be

$$
C=0.996 \pm 0.003
$$

The value of the $\chi^{2}$ per degree of freedom is around two at the optimum fit. Again the dominant source of systematic uncertainty arises from allowing the $\chi^{2}$ of the fit to increase by two. The other sources of uncertainty were investigated and determined to be negligible.

\subsubsection{Studies Using $\mathrm{J} / \Psi \rightarrow \mu^{+} \mu^{-}$Events}

The tuning of the value of $B$ and a cross-check of the value of $C$ was performed using $\mathrm{J} / \Psi \rightarrow \mu^{+} \mu^{-}$events. A sample of $\mathrm{J} / \Psi \rightarrow \mu^{+} \mu^{-}$was selected using the following requirements

- the presence of two muons, each matched to a central track.

- $p_{t}>1.5 \mathrm{GeV}$ for both muons.

- Both muons lie inside the acceptance of the muon system as defined in Section 6.1.1.

The peak obtained was fitted with a Gaussian to represent the signal and a first order polynomial to represent background. This peak is shown in Figure 8.3 producing a Gaussian with a mean of $3.0726 \pm 0.0009 \mathrm{GeV}$ and a width of $71.8 \pm 1.4 \mathrm{MeV}$.

A series of $\mathrm{J} / \Psi \rightarrow \mu^{+} \mu^{-}$events were generated using PMCS and PYTHIA with different values of $B$ and $C$. These were passed through the event selection described above. The mean and widths of the peaks were obtained by fitting with a single Gaussian. They were then compared with those seen in data. 

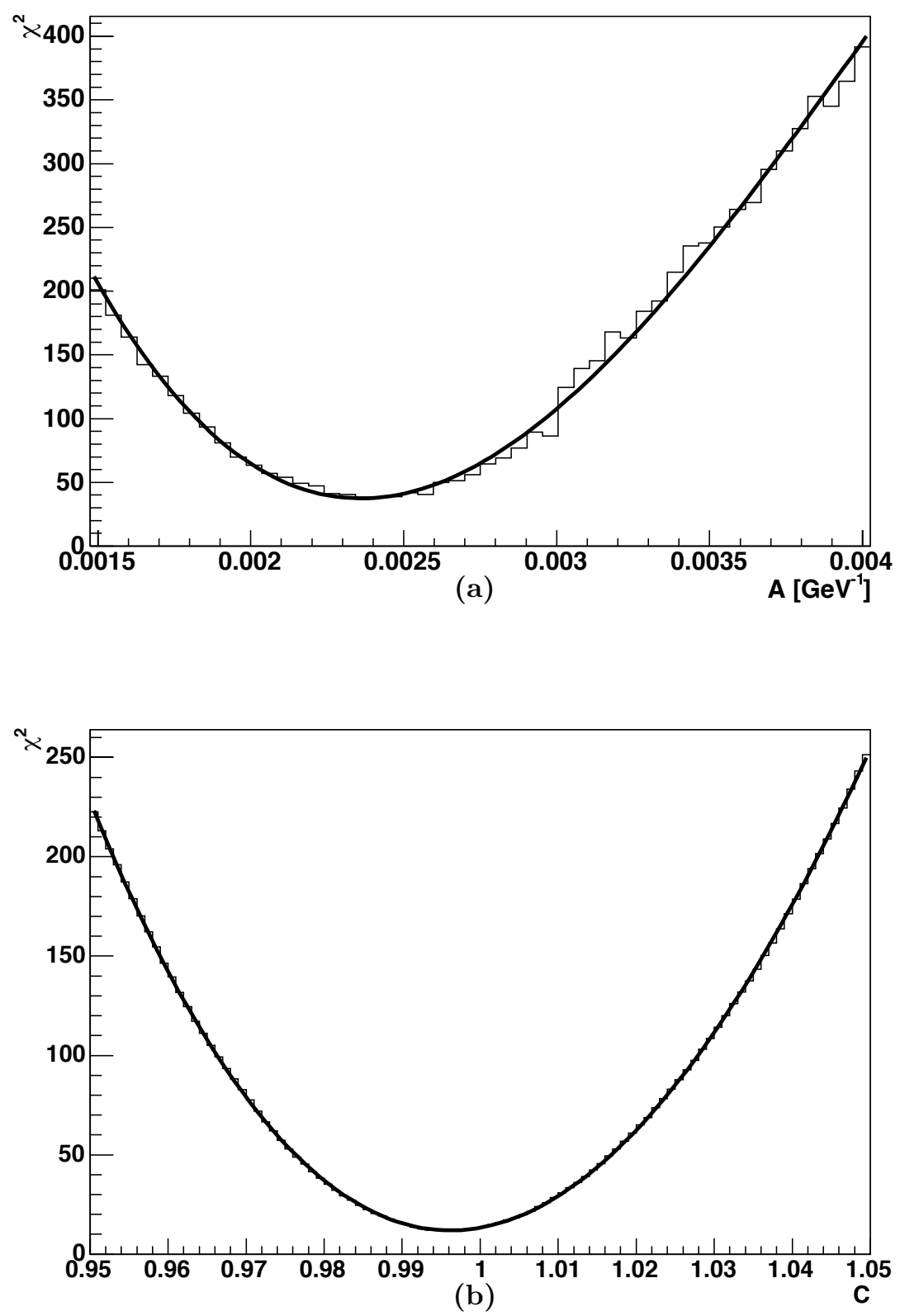

Figure 8.2: $\chi^{2}$ comparison of $\mathrm{Z} \rightarrow \mu^{+} \mu^{-}$peak between data, with the $\mathrm{W} \rightarrow \mu \nu$ track quality cuts applied, and PMCS with varying values of $A$ in the top plot and $C$ in the bottom plot . 


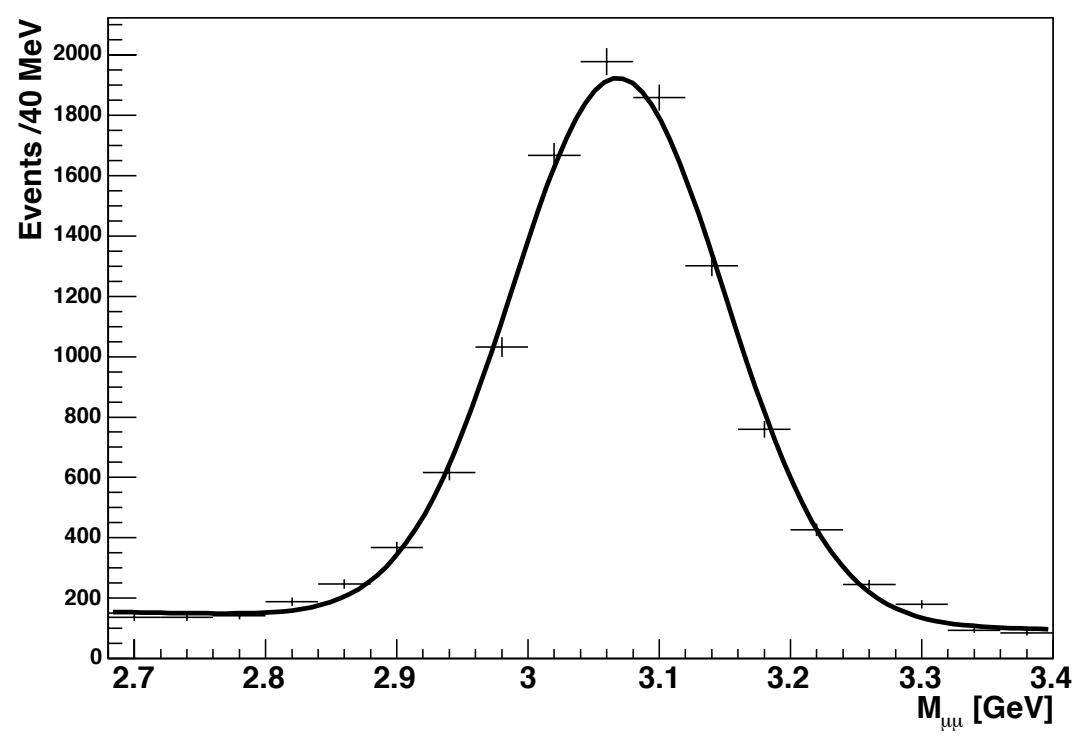

Figure 8.3: J/ $\Psi \rightarrow \mu^{+} \mu^{-}$peak fitted with Gaussian plus first order Polynomial.

The variation of the mean with $B$ is shown in Figure 8.4(a) and the variation with $C$ is shown in Figure 8.4(b). The bands represent the mean obtained from data and the uncertainty associated with this value. It is observed that the mean is relatively insensitive to the variation in $B$.

Similarly the variation of the width with $B$ is shown in Figure 8.5(a) and the variation of the width with $C$ is shown in Figure 8.5(b). It is observed that the variation of the width with $C$ is small enough to be neglected.

The procedure adopted was to fit a first order polynomial to the variation and read off where this polynomial intercepts the mean and width from data. The uncertainty is quoted as the points where this linear fit intercepts the error bars of width and mean.

By examination of Figure 8.5(a), $B$ was determined to be 

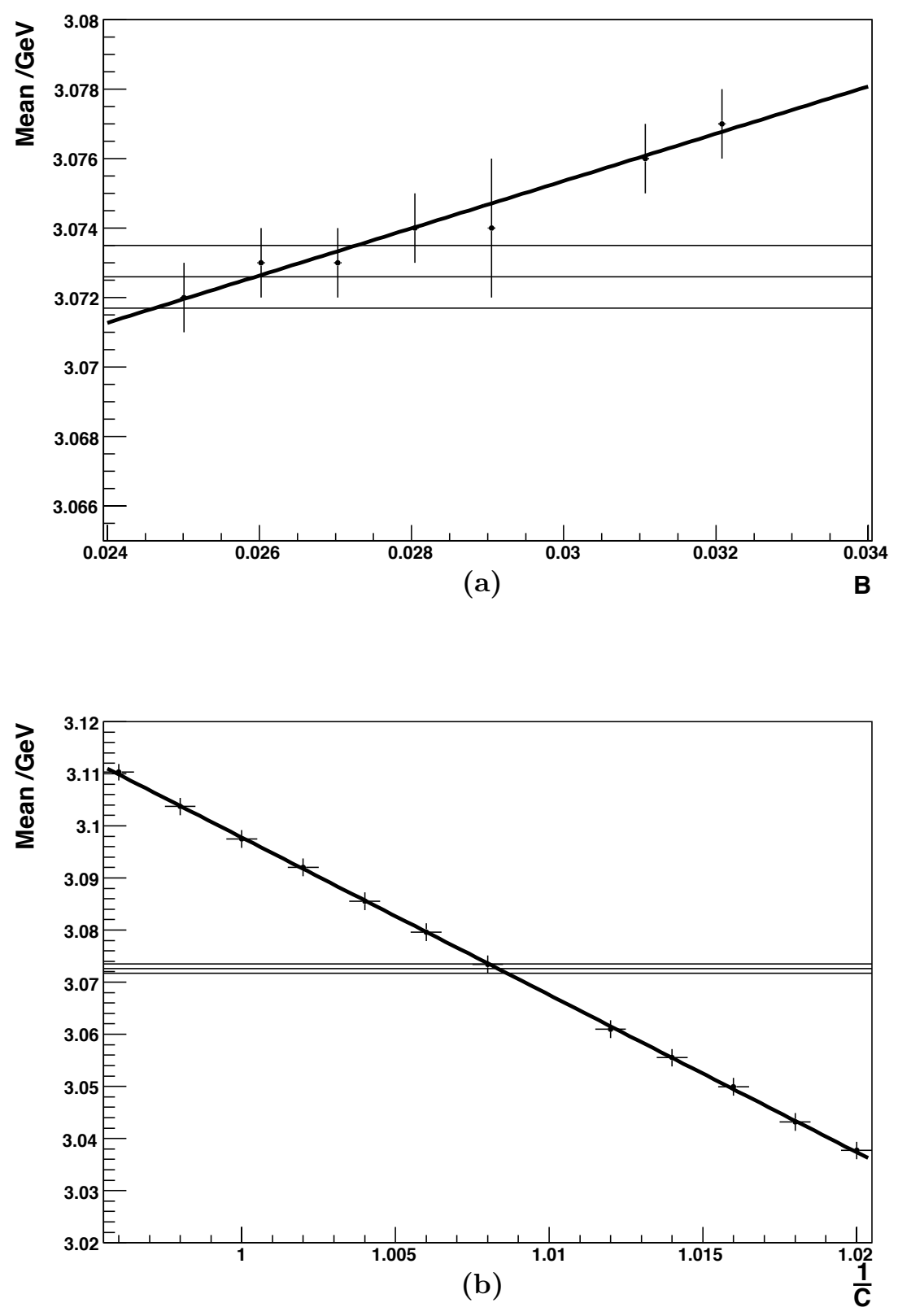

Figure 8.4: Variation of by the fitted mean of the $\mathrm{J} / \Psi \rightarrow \mu^{+} \mu^{-}$peak in PMCS with a) $B$ and b) $\frac{1}{C}$. 

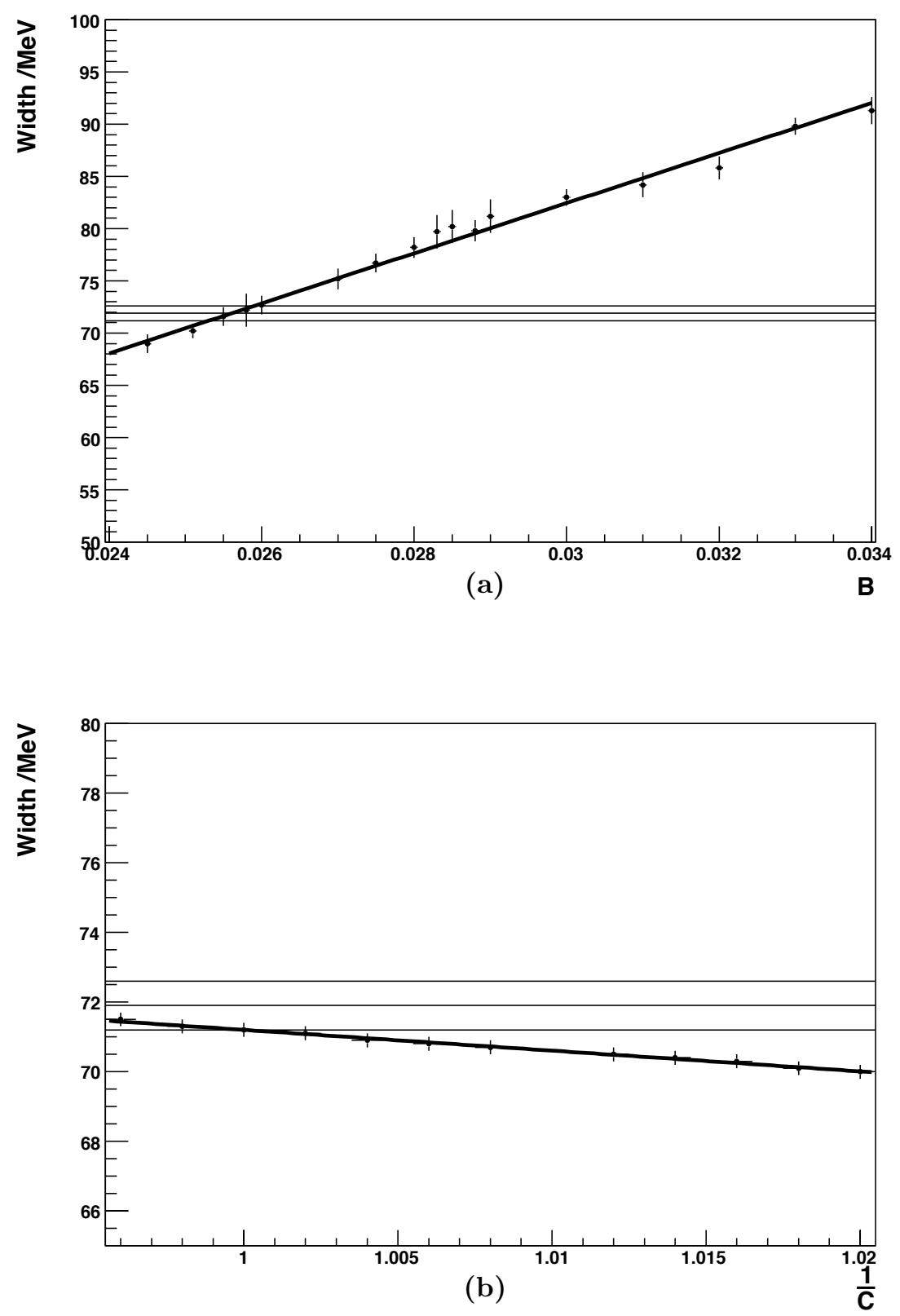

Figure 8.5: Variation of by the fitted width of the $\mathrm{J} / \Psi \rightarrow \mu^{+} \mu^{-}$peak in PMCS with a) $B$ and b) $\frac{1}{C}$. 


\begin{tabular}{|l|c|}
\hline Parameter & Value \\
\hline$A\left(\mathrm{Z} \rightarrow \mu^{+} \mu^{-}\right)$ & $0.00292 \pm 0.0010 \mathrm{GeV}^{-1}$ \\
$A(\mathrm{~W} \rightarrow \mu \nu)$ & $0.00235 \pm 0.00010 \mathrm{GeV}^{-1}$ \\
$B$ & $0.0254 \pm 0.0008$ \\
$C\left(\mathrm{Z} \rightarrow \mu^{+} \mu^{-}\right)$ & $0.992 \pm 0.003$ \\
$C(\mathrm{~W} \rightarrow \mu \nu)$ & $0.996 \pm 0.003$ \\
\hline \hline
\end{tabular}

Table 8.1: Parameters used in track resolution.

$$
B=0.0254 \pm 0.0008 \text {. }
$$

The dominant uncertainty in the determination of the value of $B$ is caused by uncertainty in the value of $A$. This is not unexpected as many $\mathrm{J} / \Psi \rightarrow \mu^{+} \mu^{-}$events are boosted in $p_{t}$ producing higher $p_{t}$ muons, increasing the sensitivity to the value of $A$. By examination of Figure 8.4(b), $C$ was determined to be 0.992 , in good agreement with the value found by tuning to the $\mathrm{Z} \rightarrow \mu^{+} \mu^{-}$peak.

The value of $B$ was not retuned for the $\mathrm{W} \rightarrow \mu \nu$ analysis. This was because the sample of $\mathrm{J} / \Psi \rightarrow \mu^{+} \mu^{-}$events did not have all the requisite variables stored to make cuts on the track quality. This is not expected to be a problem as the track quality cuts should only have a small impact on this parameter and this parameter has a negligible effect on the high $p_{t}$ physics analyses performed within this thesis.

\subsubsection{Summary of the Tuned Parameters}

The optimum values are summarised in Table 8.1. The values of $A$ and $C$ are shown with and without the tighter track quality requirements used by the $\mathrm{W} \rightarrow \mu \nu$ analysis. There is only one value of $B$, as this was assumed to be independent of these requirements. 


\begin{tabular}{|l|c|}
\hline Efficiency & Co-ordinates \\
\hline$\varepsilon_{\text {track }}$ & $\eta_{c f t}, z$ \\
$\varepsilon_{\text {L3track }}$ & $\eta_{c f t}$ \\
$\varepsilon_{\text {loose }}$ & $\eta_{A}$, in/out octant boundaries \\
$\varepsilon_{\text {L1scint }}$ & $\eta_{A}$ and $\phi_{A}$ projections \\
$\varepsilon_{L 2}$ (WAMUS) & $\eta_{A}$ and $\phi_{A}$ projections \\
$\varepsilon_{L 2}$ (FAMUS) & $\eta_{A}$ in/out octant boundaries \\
$\varepsilon_{\text {Muon }}$ (FAMUS) & $\eta_{A}$ and $\phi_{A}$ \\
\hline \hline
\end{tabular}

Table 8.2: Co-ordinates used to input efficiencies into PMCS. L2 denotes all Level 2 and Level 1 wires efficiencies. The 'muon' efficiency is the combined medium, L1scint, L1wires and L2M3 efficiency used in the $\mathrm{W} \rightarrow \mu \nu$ analysis.

As well as producing the optimum value for these parameters as used in the analysis described in this thesis this section also defines the standard procedure for tuning these parameters. This will allow a quick retuning in future.

\subsection{Modelling of Efficiencies}

This section describes the introduction of the tracking, muon identification and trigger efficiencies into PMCS, expanding on the overview given in Section 4.3. These efficiencies are measured from data, using the tag and probe method, as described in Section 4.3.2. The tag and probe method can introduce biases when efficiencies are averaged over variables to which the efficiency is sensitive. To avoid these biases the efficiency has to be measured as a function of these variables. The variables chosen are summarised in Table 8.2 [36]. The subscript on the angular co-ordinates indicates where in the detector they are calculated, with $A$ indicating that they are determined at the 'A' layer of the muon chambers and CFT indicating that they are calculated at the outer layer of the CFT. 


\subsubsection{Tracking Efficiencies}

There are two sets of tracking efficiency included into PMCS. The first is the probability for a track to be identified and reconstructed by the tracking algorithm. This was the efficiency used by the $\mathrm{Z} / \gamma^{*} \rightarrow \mu^{+} \mu^{-}$analysis. The other option requires the track to pass the quality requirements made by the $\mathrm{W} \rightarrow \mu \nu$ analysis. The user of PMCS can specify either option before starting to generate events.

The efficiencies are added as look up tables, with entries for each combination of $\eta_{c f t}$ and $z$. Look up tables store the efficiency as an array of numbers with each component of the array corresponding to the efficiency of a region of the detector. The co-ordinates are noted for each track and the appropriate efficiency 'looked up' from this array. A random number is then generated with uniform probability between zero and one. If the random number is less than the efficiency then the track is considered to have been detected, else it is considered not to have been found. This Boolean value is stored in the output, alongside all the other information on the track, such as smeared $p_{t}$.

To confirm that these efficiencies have been correctly added to the simulation a series of cross-checks were performed. In one of these a series of $\mathrm{Z} / \gamma^{*} \rightarrow \mu^{+} \mu^{-}$ events are generated and passed through the simulation. The tracking efficiency is then 'measured' in this sample in the same bins in $\eta_{c f t}$ and $z$ and compared to the input efficiency. One of these comparison plots is shown in Figure 8.2.1. This displays the input and output tracking efficiency from PMCS, which agree well.

\subsubsection{Muon Efficiencies}

There are three sets of muon identification efficiencies included into the simulation:

1. The muon is identified as of at least loose quality; 


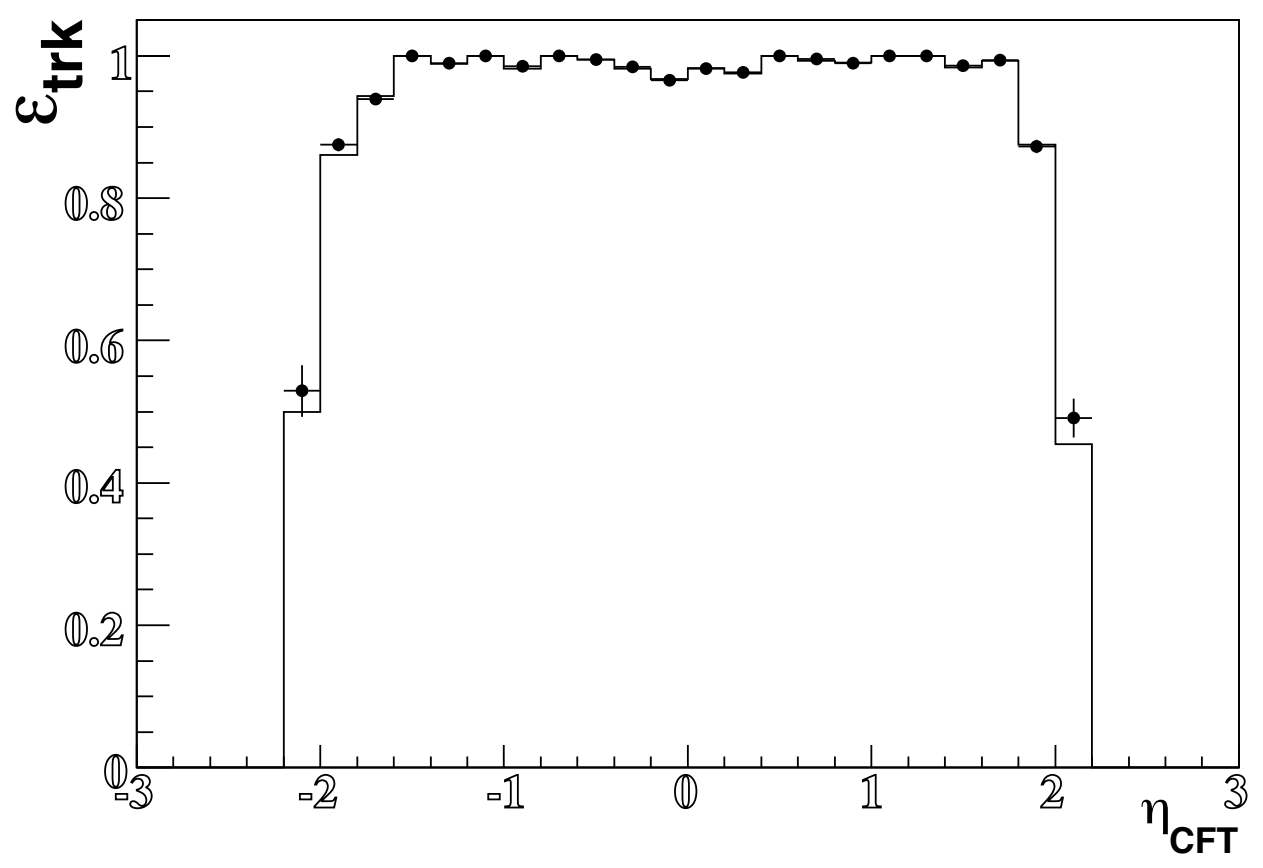

Figure 8.6: Cross-check to ensure that the tracking efficiency is correctly added into PMCS. This is shown, as a function of $\eta_{c f t}$, for tracks in the region $-10<z<10 \mathrm{~cm}$. The line are the input efficiencies measured from data and the points with error bars is the output efficiency measured from PMCS.

2. The muon is identified as of at least medium quality;

3. The muon is identified as of at least medium quality given that it is identified as of at least loose quality.

The first is used for the $\mathrm{Z} / \gamma^{*} \rightarrow \mu^{+} \mu^{-}$analysis, the second was used in studies for the $\mathrm{W} \rightarrow \mu \nu$ analysis. The last is used to allow the correlations between the $\mathrm{Z} / \gamma^{*} \rightarrow \mu^{+} \mu^{-}$and $\mathrm{W} \rightarrow \mu \nu$ cross sections in the measurement of their ratio. PMCS outputs both the loose and medium efficiencies as Boolean outputs for each muon. The user can choose whether to use the measurement of the medium efficiency or the medium efficiency with respect to the loose efficiency. The latter allows comparisons 
to be made more accurately between the loose and medium efficiencies, but has a higher statistical uncertainty associated with it.

The efficiencies are modelled in the same way as the tracking efficiencies. Firstly each efficiency is measured as a function of detector coordinates chosen to minimise any bias [36] and stored as look up tables. Then, using the method described in Section 4.3 .1 a random number is used to decide whether the muon is reconstructed or not. The muon trigger efficiencies are also included, and are discussed in more detail in Section 8.2.3.

The loose muon efficiency is measured separately in the FAMUS and the WAMUS. Within these regions the efficiency is added as look up tables, in $\eta_{A}$, in three different sub-regions. These sub-regions are defined by proximity to the octant boundaries in the muon chambers. The division was done by examining a plot of the muon identification efficiency versus position in the octant. The exact definitions, as given in Appendix B, are slightly arbitrary.

The medium efficiency is added as a two dimensional look up table in $\eta_{A}$ and $\phi_{A}$. The reason why this is a lot less elaborate than the loose efficiency is because it was intended for studies for the $\mathrm{W} \rightarrow \mu \nu$ analysis. Therefore, unlike in the $\mathrm{Z} / \gamma^{*} \rightarrow \mu^{+} \mu^{-}$ analysis, there is no need to worry about correlations between the muons. The medium efficiency with respect to loose is added using the same co-ordinates as the medium efficiency.

\subsubsection{Trigger efficiencies}

There are only three triggers simulated by PMCS at present. These are the dimuon trigger and single muon triggers used by the $\mathrm{Z} / \gamma^{*} \rightarrow \mu^{+} \mu^{-}$and $\mathrm{W} \rightarrow \mu \nu$ cross section measurements as described in Section 6.1.

The two analyses have different approaches, because there is required to be only 
one muon in the $\mathrm{W} \rightarrow \mu \nu$ analysis. This implies that all of the requirements of the single muon trigger have to be met by that one muon. The efficiency measured is a single efficiency, for the muon to be reconstructed as medium and to meet all the requirements of the single muon trigger, except the track trigger efficiency. This reduces the systematic uncertainty caused by limited statistics in the $\mathrm{Z} \rightarrow \mu^{+} \mu^{-}$ samples used to determine the efficiencies.

In the $\mathrm{Z} / \gamma^{*} \rightarrow \mu^{+} \mu^{-}$analysis, because of the presence of two muons, more care is required due to possible biases caused by correlations between the muons. The approach taken here is to measure the efficiency of all trigger objects, as defined in Section 3.3, and to evaluate whether these conditions have been met. The trigger condition is then assembled from these combination of trigger objects to see whether the overall trigger has been met. As they were intended for the $\mathrm{Z} / \gamma^{*} \rightarrow \mu^{+} \mu^{-}$analysis they are only evaluated for muons that meet at least the loose muon identification requirements. The two triggers modelled by the $\mathrm{Z} / \gamma^{*} \rightarrow \mu^{+} \mu^{-}$analysis are the di-muon trigger and the single muon trigger.

The di-muon trigger requires:

1. At least two muons to meet the tight scintillator requirements;

2. At least one muon to be identified by Level-2 as being of at least medium quality.

The single muon trigger requires:

1. At least one muon passes both the tight scintillator and loose wires conditions.

2. At least one muon to be identified by Level-2 as being of at least medium quality and with $p_{t}>3 \mathrm{GeV}$.

3. At least one muon is associated with a track, with $p_{t}>10 \mathrm{GeV}$, at Level-3. 
In order to model these efficiencies the following trigger objects are included.

- The 'L1scint' efficiency

This is the probability that the muon meets the tight scintillator requirement. It is measured with respect to the loose efficiency in a grid of bins of $\eta_{A}$ and $\phi_{A}$. The efficiencies are measured as one-dimensional projections in $\eta_{A}$ and $\phi_{A}$, which are multiplied together to produce the two-dimensional grid. To avoid double counting the efficiency the $\phi_{A}$ projection is normalised so that the total efficiency for $\mathrm{Z} / \gamma^{*} \rightarrow \mu^{+} \mu^{-}$events, when the efficiency is included only as a function of $\phi_{A}$, is unity. One dimensional projections were used due to limited statistics, especially at high $\eta_{A}$.

- The 'L1wires' efficiency.

This is the probability that a muon, which meets the tight scintillator requirements, meets the loose wire requirements. It is measured with respect to the 'L1scint' efficiency. In the WAMUs the efficiency is measured as twodimensional projections, like the 'L1scint' efficiency. In the FAMUs the efficiency is split into octant boundary regions, like the loose muon identification efficiency.

- The 'L2M0' efficiency

This is the probability that a muon, which meets the tight scintillator requirements, is identified by Level-2 as being of at least medium quality. The efficiency is measured using the same co-ordinates as the 'L1wires' efficiency.

- The 'L2M3' efficiency.

This is the probability that a muon, which meets the tight scintillator requirements, is identified by Level-2 as being of at least medium quality and with $p_{t}>3 \mathrm{GeV}$. It is evaluated for two cases; where the muon meets the 'L1wires' and 'L1scint' requirements and for cases where the muon fails one of these 
requirements. For the case where the muon meets these requirements the efficiency is modelled as a function of $\eta_{A}$ and $\phi_{A}$ in the wAMUS and $\eta_{A}$ alone in the FAMUS. For the case where the muon fails to meet these requirements the efficiency is measured using the same co-ordinates as the 'L1wires' efficiency.

- The 'L3TK' efficiency.

This is the probability that the muon is associated with a track, of at least $10 \mathrm{GeV}$, by Level-3. The 'L3TK' efficiency is measured with respect to the tracking efficiency. There are two sets of efficiencies, one with respect to the default efficiency and the other with respect to the tighter track quality requirements made by the $\mathrm{W} \rightarrow \mu \nu$ analysis. Both are added in terms of $\eta_{c f t}$ of the track. Which set of track trigger efficiencies employed is determined by the choice of tracking efficiency above.

The overall trigger efficiency is then assembled from these trigger objects. The modelling of the trigger efficiency for the $\mathrm{Z} / \gamma^{*} \rightarrow \mu^{+} \mu^{-}$analysis is thus a complicated procedure. As a cross-check all events that pass the $\mathrm{Z} / \gamma^{*} \rightarrow \mu^{+} \mu^{-}$event selection are broken down into three categories:

- Events that pass both the single muon and di-muon triggers;

- Events that pass only the single muon trigger and fail the di-muon trigger;

- Events that pass only the di-muon trigger and fail the single muon trigger.

Figure 8.7 shows $\eta_{A}$ of the muons for events in these three categories. The top plot is filled with muons from the first category, the middle plot with events from the second and the lower plot with events from the third. All plots use the same normalisation which is calculated from the ratio of the total number of events passing all the selection criteria. The good agreement between both the shapes and the relative normalisations demonstrates that the simulation of the trigger efficiency is reliable. 

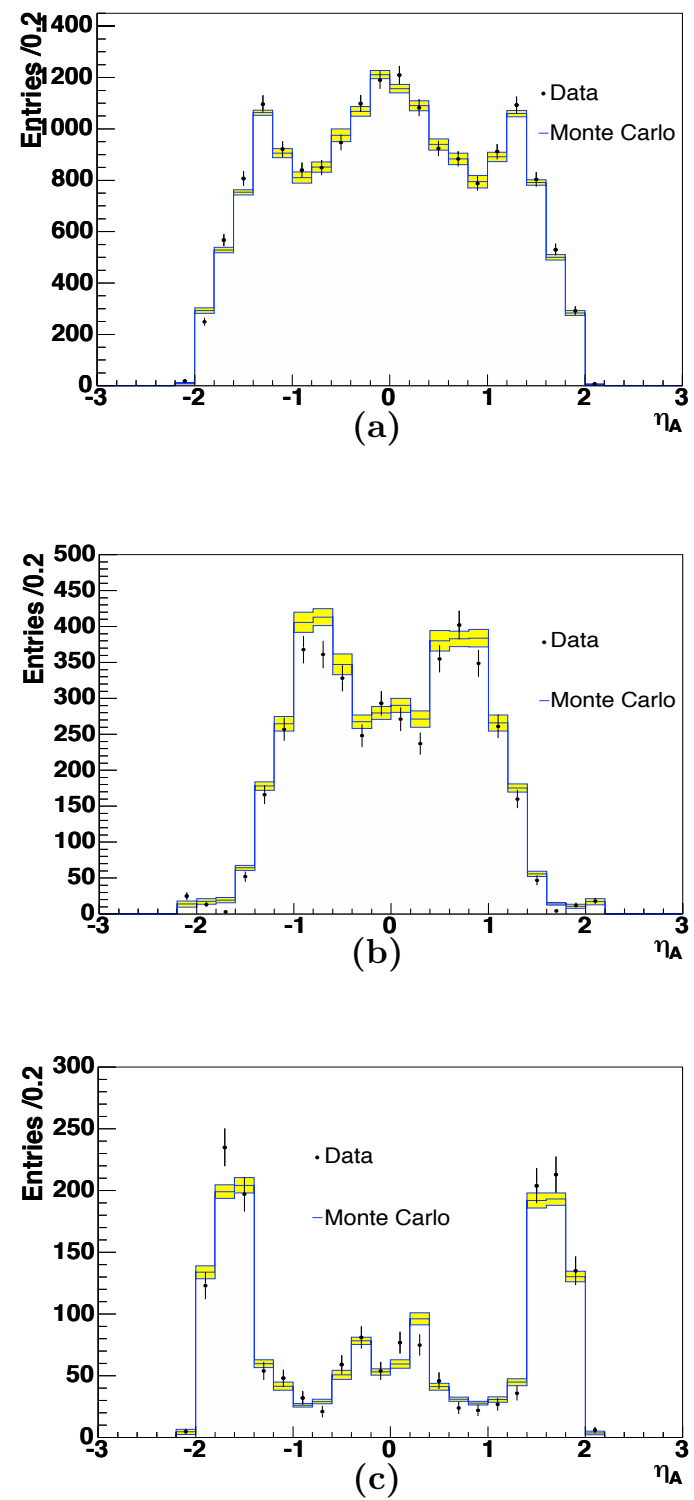

Figure 8.7: The distribution of the muon $\eta_{A}$ for the three different trigger combinations. The data is shown as the points with error bars. The PMCS prediction is shown as blue histograms. 


\subsubsection{Uncertainty on Observables}

The tracking, muon identification and trigger efficiencies, as measured using the tag and probe method, have an uncertainty associated with them. This is due to limited statistics in the samples of $\mathrm{Z} / \gamma^{*} \rightarrow \mu^{+} \mu^{-}$used to determine the efficiencies. This section describes a method used to propagate this uncertainty to a systematic uncertainty on an observable, such as an acceptance. This uncertainty is often denoted the systematic statistical, or syst-stat, uncertainty.

Each efficiency in the look up tables has an uncertainty associated with it. When the syst-stat uncertainty is evaluated each of these efficiencies are fluctuated. This fluctuation is performed according to a series of Gaussian distributions, one for each value in the tables. The mean of each Gaussian is at the central value of each efficiency. The width of each Gaussian is equal to the uncertainty of each efficiency. The observable is then calculated using this new, fluctuated look up table. This process is repeated many times and the standard deviation on the observable calculated. The syst-stat uncertainty is taken to be equal to this standard deviation.

This method has been used in both the $\mathrm{W} \rightarrow \mu \nu$ and $\mathrm{Z} / \gamma^{*} \rightarrow \mu^{+} \mu^{-}$analyses and has been adopted by the $\mathrm{W} \rightarrow e \nu$ and $\mathrm{Z} \rightarrow e^{+} e^{-}$analyses amongst others [39]. Figure 8.8 shows the spread in the value of $R$ as used to calculate the syst-stat uncertainty in Section 9.2.1.

\subsection{Applications of PMCS}

This section describes measurements made using PMCS. Section 8.3.1 gives a detailed description of $\epsilon_{\mathrm{MC}}$, the efficiency of the $\mathrm{Z} / \gamma^{*} \rightarrow \mu^{+} \mu^{-}$event selection. Section 8.3.2 gives brief overviews of other analyses using PMCS. These examples are chosen to illuminate the various uses of electroweak bosons as described in Chapter 5. 


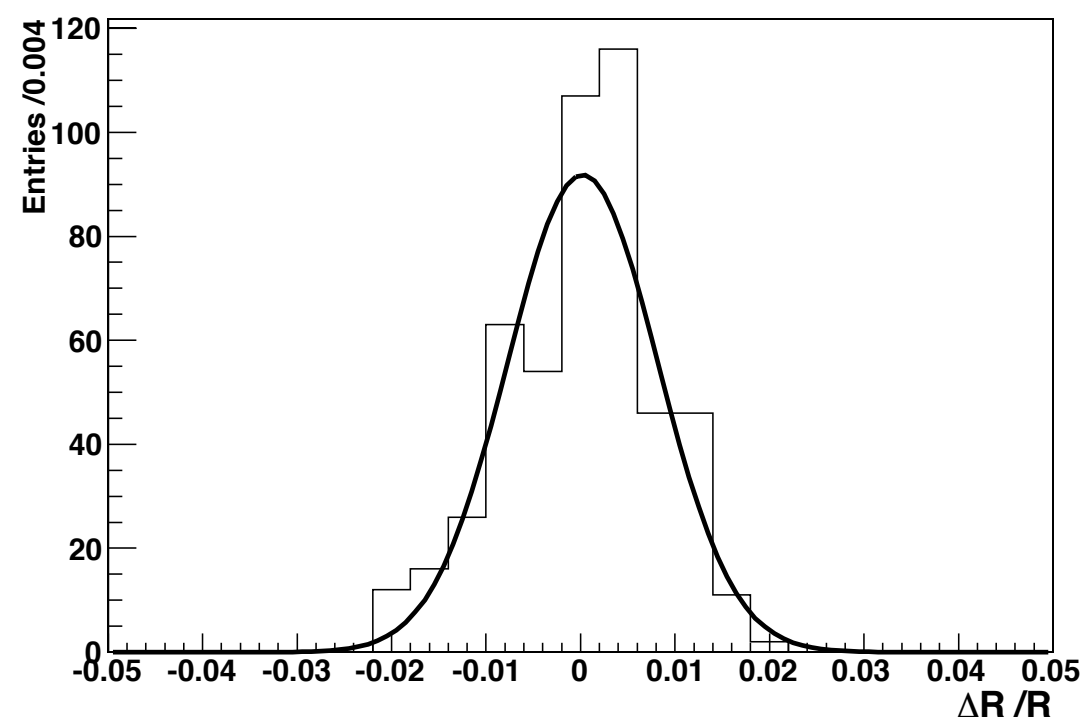

Figure 8.8: The spread in values of $R$ as evaluated in Section 9.2.1.

\subsubsection{Efficiency of Z $/ \gamma^{*} \rightarrow \mu^{+} \mu^{-}$Event Selection}

This section describes the evaluation of the efficiency of the $\mathrm{Z} / \gamma^{*} \rightarrow \mu^{+} \mu^{-}$event selection, $\epsilon_{\mathrm{MC}}$. The central value is the same as the number used in [35] although some of the systematic uncertainties have been updated for this analysis. This number is used in the calculation of the $\mathrm{Z} / \gamma^{*} \rightarrow \mu^{+} \mu^{-}$cross section in Chapter 6 .

The efficiency $\epsilon_{\mathrm{MC}}$ was calculated using a series of events, generated with the PYTHIA generator and the CTEQ 6.1 NLO PDF sets and passed through the PMCS simulation. The values of the parameters used to describe the parton shower in PYTHIA were tuned to get reasonable agreement between the boson $p_{t}$ distribution in data and PMCS [59]. The parameters used to describe the underlying soft interaction are those of "tune A" [60].

Although the $\mathrm{Z} / \gamma^{*} \rightarrow \mu^{+} \mu^{-}$cross section is measured only for di-muon masses greater than $40 \mathrm{GeV}$ events are generated from $30 \mathrm{GeV}$ upwards. This is to account 


\begin{tabular}{|l|c|}
\hline Period & $\epsilon_{\mathrm{MC}}$ \\
\hline 1 & 0.239 \\
2 & 0.268 \\
3 & 0.322 \\
\hline \hline
\end{tabular}

Table 8.3: $\epsilon_{\mathrm{MC}}$ as evaluated for the three data taking periods of the $\mathrm{Z} / \gamma^{*} \rightarrow \mu^{+} \mu^{-}$ analysis. The statistical uncertainty is negligible. The systematic uncertainty is evaluated in detail in Section 8.3.1

for events that have generated di-muon mass less than $40 \mathrm{GeV}$, but which are reconstructed as having a di-muon mass greater than $40 \mathrm{GeV}$. The efficiency, $\epsilon_{\mathrm{MC}}$, is defined to be the number of events selected divided by the number of events generated with mass greater than $40 \mathrm{GeV}$. This efficiency was determined separately for the three data taking periods, summarised in Table 8.3.

Figures 8.9 to 8.13 show a series of data PMCS comparison plots, to demonstrate that the PMCS simulation reproduces data well. Figure 8.9 shows the invariant mass distribution for all data taking periods combined. The contribution from background is shown as the filled red histogram, which tends to be concentrated at lower masses. It can be observed that the data and PMCS distributions agree well in this low mass region, demonstrating that the background is well understood.

Figure 8.10 shows the $p_{t}$ distribution of the muons for all selected events to demonstrate that the $p_{t}$ resolution of the tracking system is well modelled. The $p_{t}$ distribution for the muon with the highest $p_{t}$ in the event and the muon with the lowest $p_{t}$ in an event are displayed separately. For these plots only one of the muons is required to pass the $p_{t}$ cut, to allow the region near the $p_{t}$ cut of $15 \mathrm{GeV}$ to be examined. It can be observed that the data and PMCS simulation agree well in this range. The discrepancy at low $p_{t}$ values in Figure $8.10(\mathrm{~b})$ is due to contamination from background processes. 


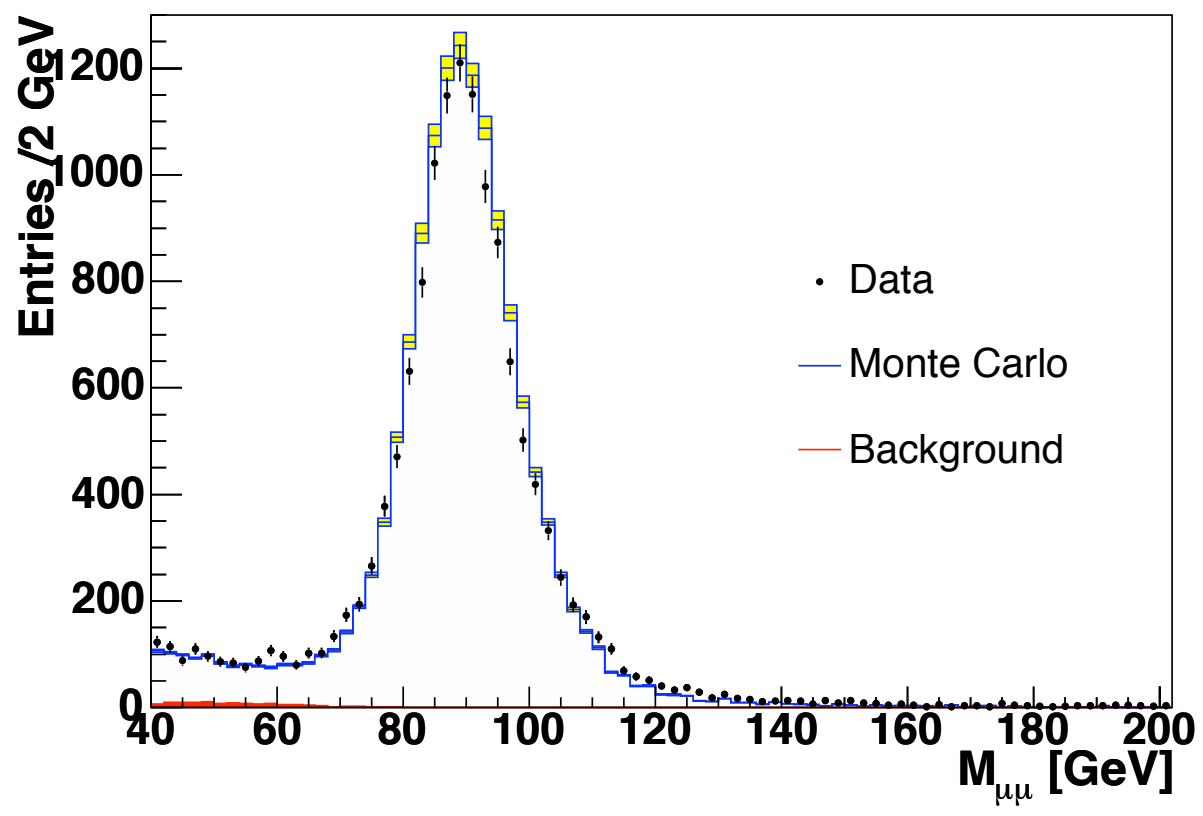

Figure 8.9: Di-muon invariant mass distribution for all three data taking periods combined. The data is the points with error bars. The blue histogram is the PMCS prediction with yellow bands denoting the syst-stat uncertainty. The contribution from backgrounds is shown as the filled red histogram. 

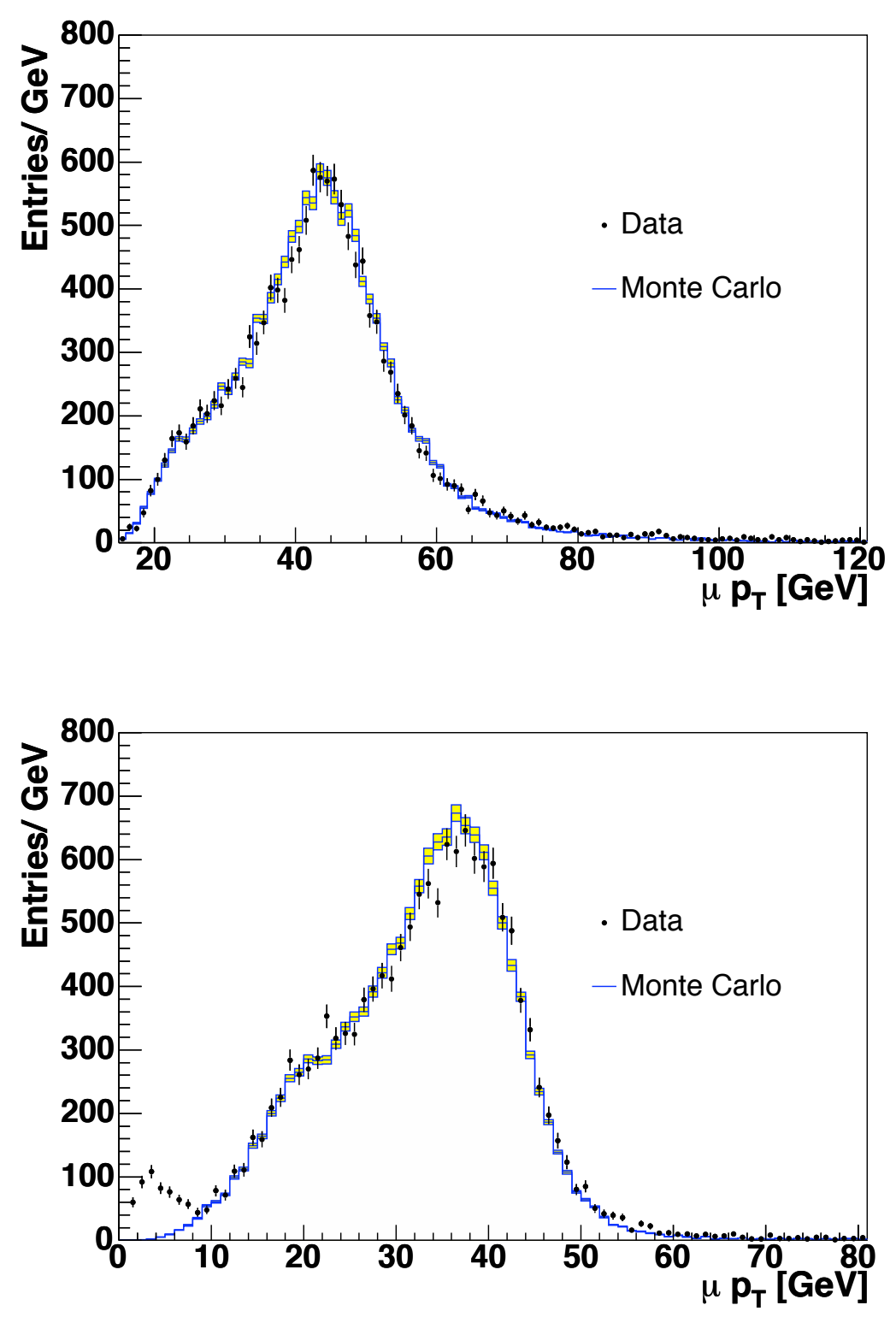

Figure 8.10: The distribution of the muon $p_{t}$ with all selection cuts except the $p_{t}$ cut on the muon with the lower amount of $p_{t}$ in the event. The data is shown as the points with error bars. The PMCS prediction is shown as blue histograms with yellow bands for the syst-stat uncertainty. The upper histogram shows this for the muon in the event with the higher $p_{t}$. The lower plot shows this for the muon in the event with the lower $p_{t}$. 
Figures 8.11 and 8.12 are included to demonstrate that the angular dependence of the tracking, muon identification and trigger efficiencies are well described. Figure 8.11 shows the $\eta_{A}$ distribution for selected events in the first and last data taking periods. The increase in efficiency at $\left|\eta_{A}\right|$ around one resulting from selecting events that meet the single muon trigger requirements can be seen in Figure 8.11(b). Figure 8.12 shows the $\phi_{A}$ distribution for selected events in the first and last data taking periods. The compromised region of the muon chambers can be observed between $\phi_{A}=4.25$ and 5.15 rads. The correlations, in $\phi$, between the two muons produces the drop in efficiency $\pi$ rads from this region.

Figure 8.13 shows the rapidity distribution of the $Z / \gamma^{*}$ for all selected events. In addition to being sensitive to the angular modelling of efficiencies, this distribution is affected by the PDF used. The good agreement shown in Figure 8.13 demonstrates that both these factors are modelled well.

The default set of parameters used by PYTHIA are known to give poor agreement between the $p_{t}$ of the $\mathrm{Z}$ boson in data and Monte Carlo [59]. Figure 8.14 shows the

$p_{t}$ distribution of the $Z / \gamma^{*}$ for all selected events, demonstrating that the set of parameters used in this thesis provides good agreement between data and Monte Carlo.

\section{Evaluation of Systematic Uncertainties on $\epsilon_{\mathrm{MC}}$}

This section describes the evaluation of the uncertainty on $\epsilon_{\mathrm{MC}}$. The sources of systematic uncertainty are summarised in Table 8.4. The uncertainty due to choice of PDF was evaluated, using the DØ prescription as described in Chapter 7, to be $+1.2 \% /-1.1 . \%$. The syst-stat uncertainty was evaluated following the prescription described in Section 8.2.4. This uncertainty was determined to be $2.5 \%$ in the first data taking period, $0.9 \%$ in the second and $0.6 \%$ in the third.

The next most significant source of uncertainty was obtained by evaluating the 

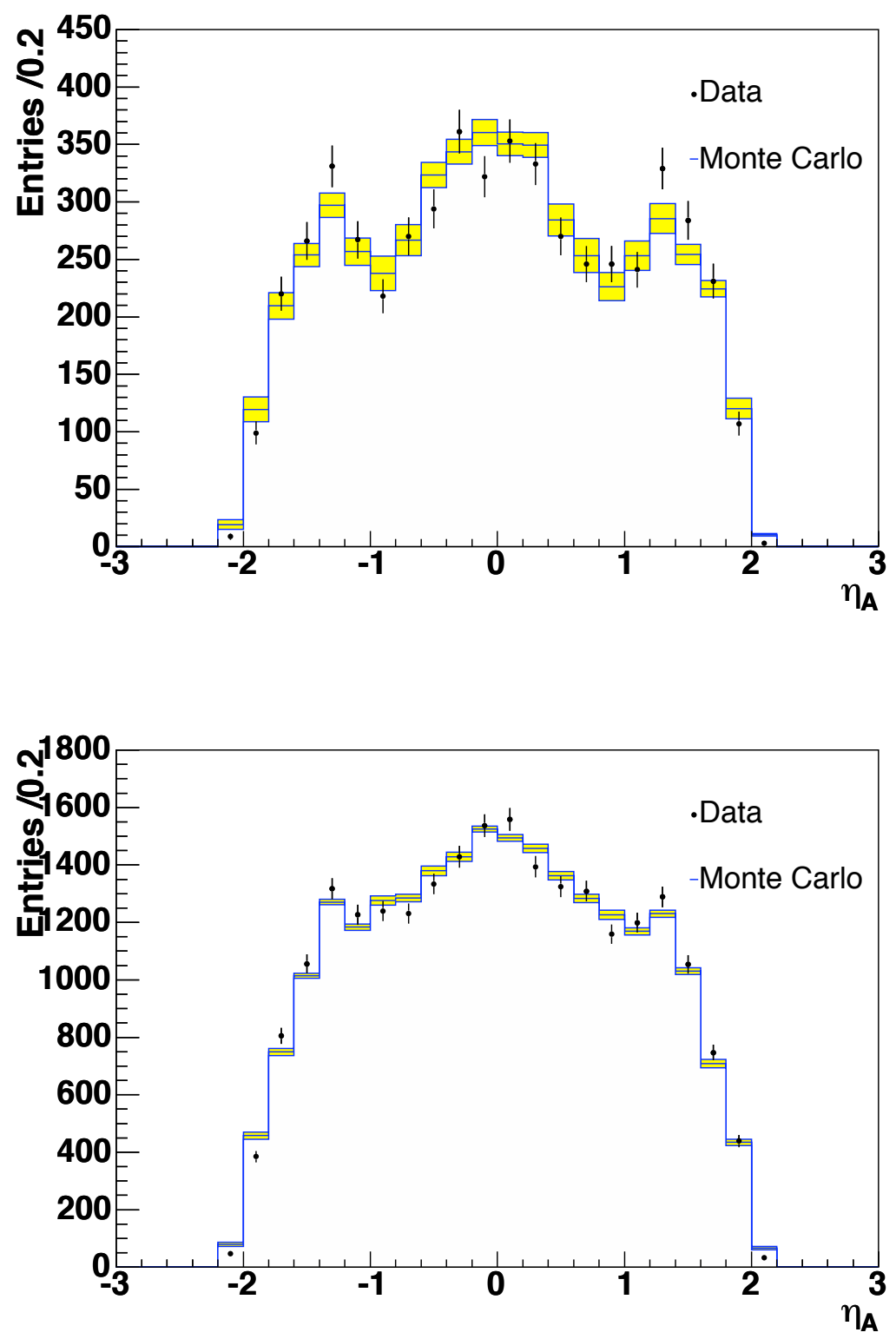

Figure 8.11: The distribution of the muon $\eta_{A}$. The data is shown as the points with error bars. The PMCS prediction is shown as blue histograms with yellow bands for the syst-stat uncertainty. The upper histogram shows this for the first data taking period, the lower for the last data taking period. 

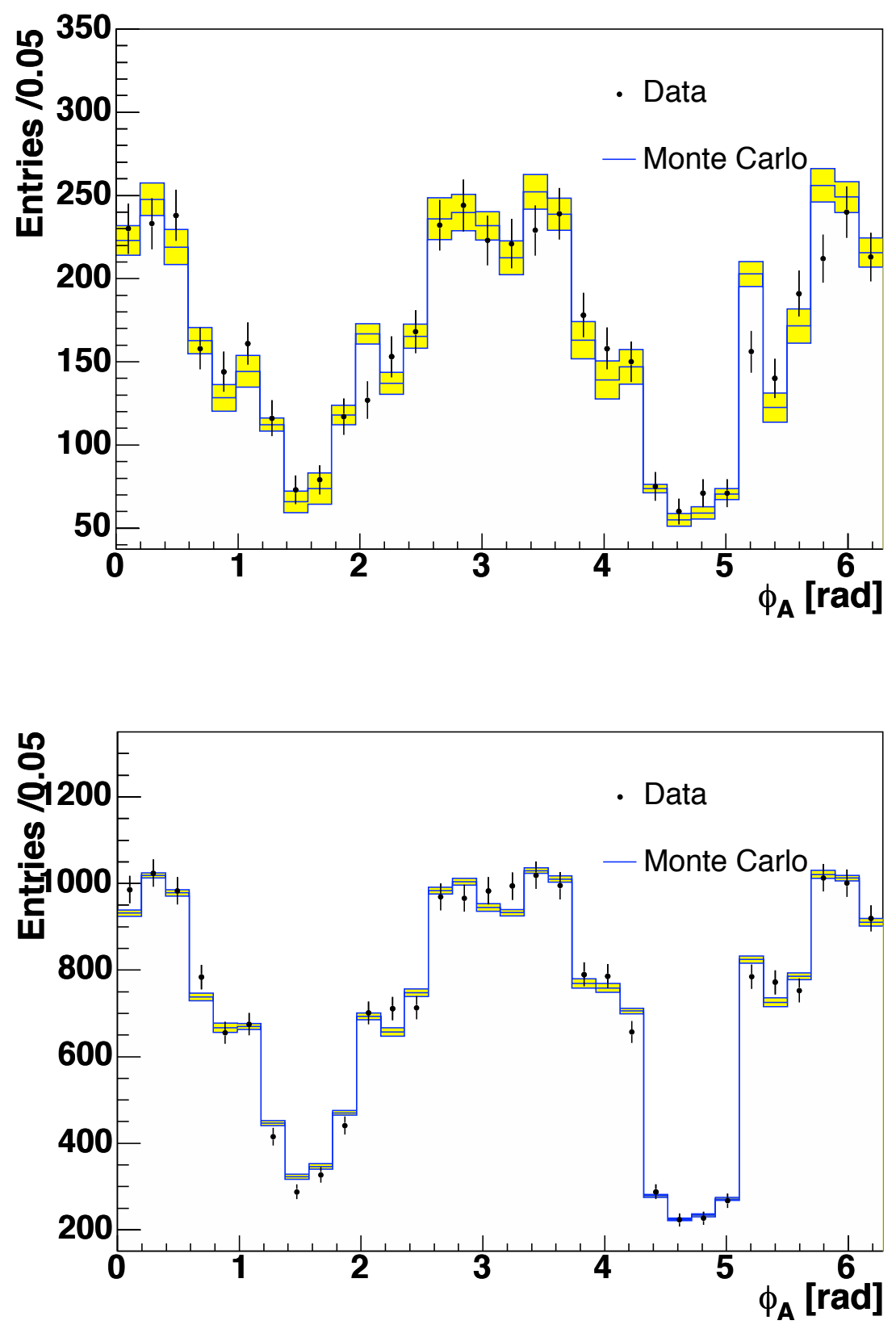

Figure 8.12: The distribution of the muon $\phi_{A}$. The data is shown as the points with error bars. The PMCS prediction is shown as blue histograms with yellow bands for the syst-stat uncertainty. The upper histogram shows this for the first data taking period, the lower for the last data taking period. 

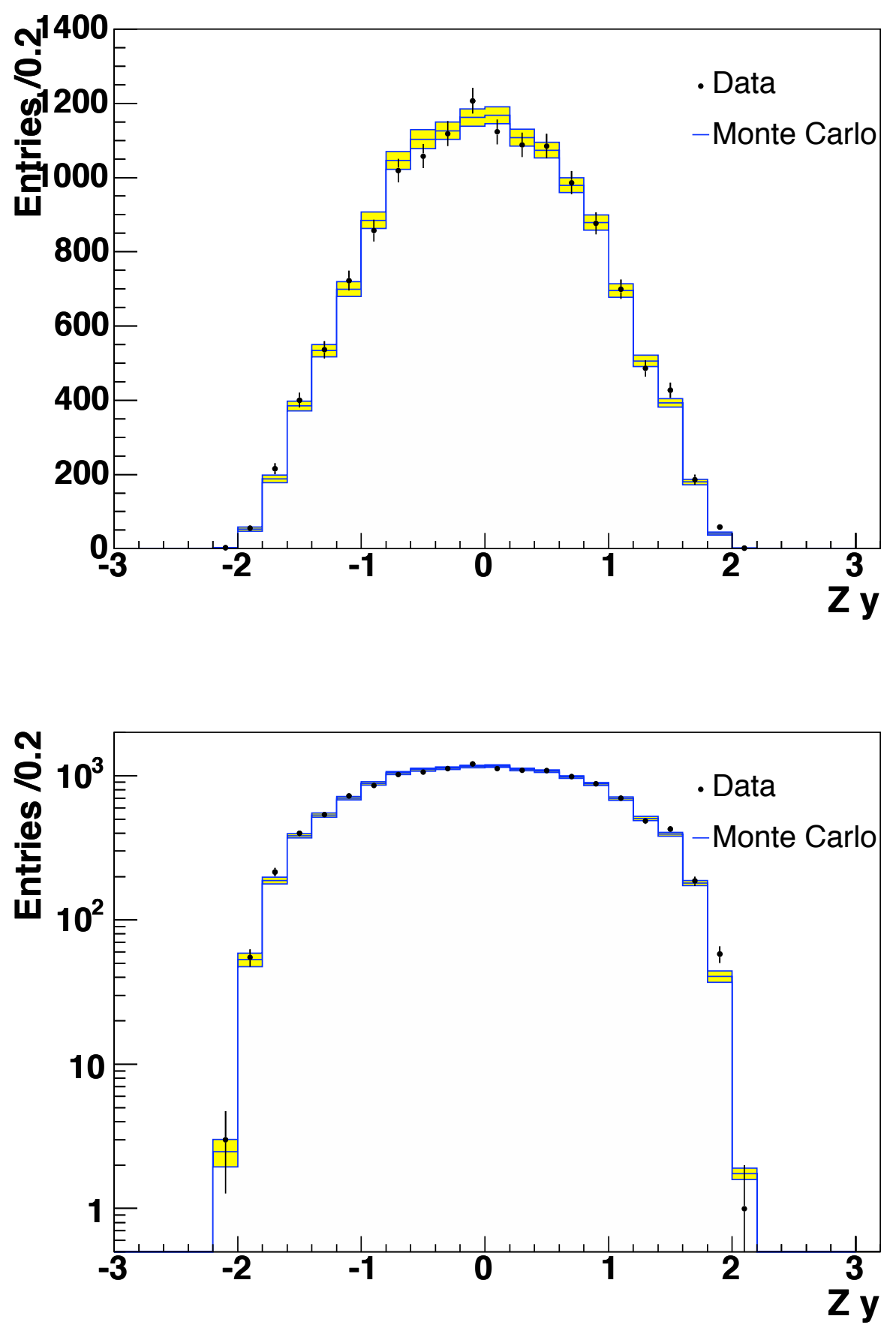

Figure 8.13: The distribution of the $\mathrm{Z}$ boson $p_{t}$. The data is shown as the points with error bars. The PMCS prediction is shown as blue histograms with yellow bands for the syst-stat uncertainty. The upper histogram shows this on a standard scale, the lower on a logarithmic scale. 

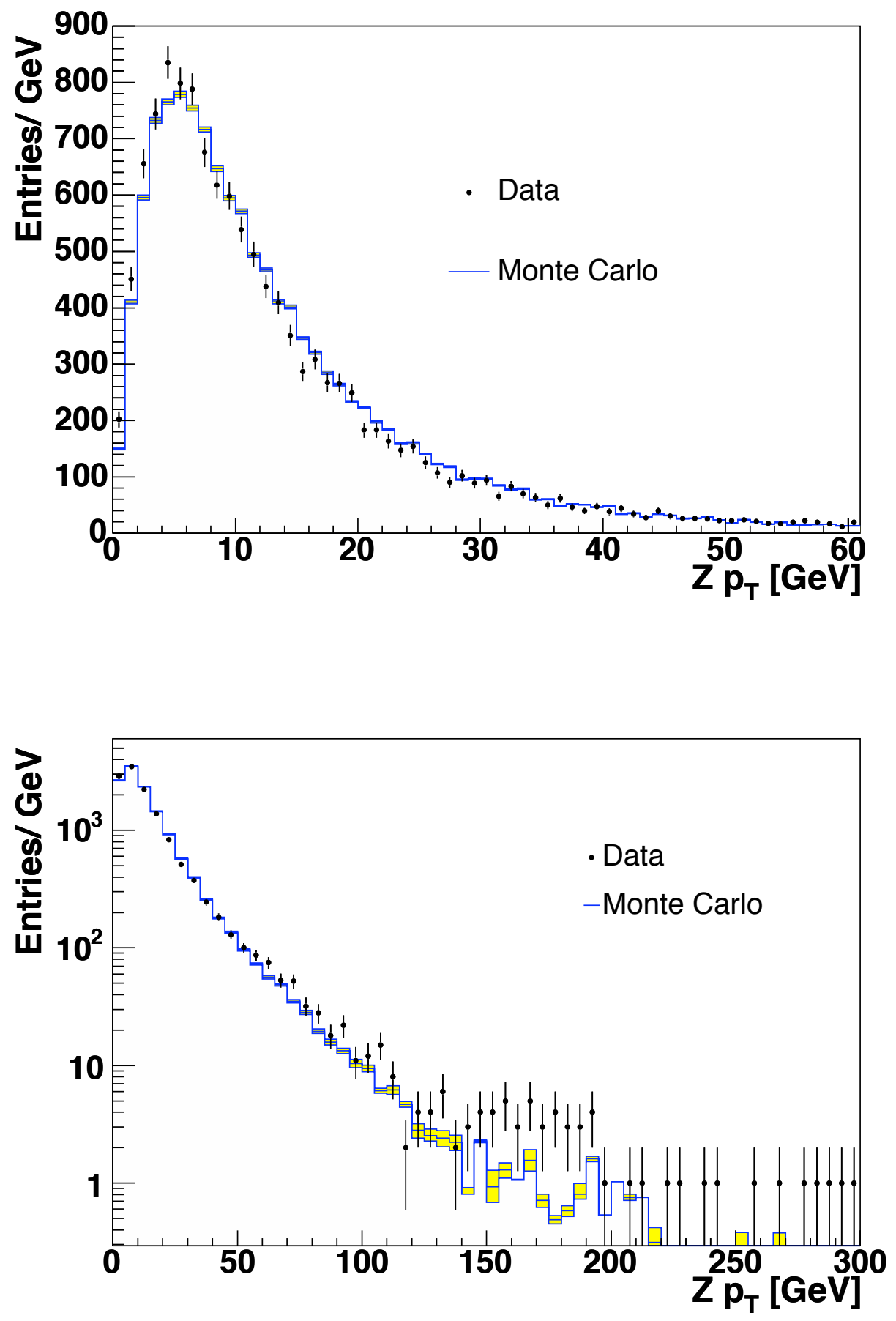

Figure 8.14: The distribution of the $\mathrm{Z}$ boson $p_{t}$. The data is shown as the points with error bars. The PMCS prediction is shown as blue histograms with yellow bands for the syst-stat uncertainty. The upper histogram shows this on a standard scale, the lower on a logarithmic scale. 
efficiency using the DMCS simulation and comparing the result with that obtained by PMCS. As the tracking and muon identification efficiencies are modelled poorly by the DMCS simulation a correction factor has to be applied. This resulting efficiency differs by $1.1 \%$ from the efficiency found by PMCS [36]. This difference could relate to the manner in which the correction factor is introduced, which make no attempt to model the shape of the efficiency correctly. But as this could also be related to some correlation not modelled by PMCS, it is quoted as a systematic uncertainty.

A further source of uncertainty arises from the modelling of the octant boundaries in the loose muon identification efficiencies. As stated in Section 8.2.2 the definition of these boundaries is somewhat arbitrary. To make some account of this the definitions are changed by halving the size of all the boundaries. This led to a $1.0 \%$ change in the acceptance which is quoted as a systematic uncertainty.

When assessing $\epsilon_{\mathrm{MC}}$ one of the inputs is the beamspot parameterisation, as defined in Section 3.1, which is only known to a finite degree of accuracy. The central value, a Gaussian with a width of $26.7 \mathrm{~cm}$, was taken from studies performed for the $\mathrm{W} \rightarrow \mu \nu$ analysis [42]. This model slightly underestimated the number of events at high values of $|z|$, as can be seen in Figure 8.15. This difference is assumed to arise from the fact that, especially at high values of $|z|$, the beamspot distribution is not just a Gaussian [61,62]. A small correction is applied to increase the number of events at high $|z|$ and good agreement is obtained.

To estimate the systematic uncertainty caused by the lack of knowledge of the beamspot distribution different parameterisations of the beamspot are used. The first model used was the single Gaussian with width $26.7 \mathrm{~cm}$, without the corrections at high $|z|$, in which the acceptance changed by a negligible amount. The second used a single Gaussian, with width $28 \mathrm{~cm}$. This gives slightly worse agreement, but not poor enough to be excluded. The acceptance decreased by $0.6 \%$ with this model and this difference is quoted as a systematic uncertainty. The two different models are compared to the data in Figure 8.16. 


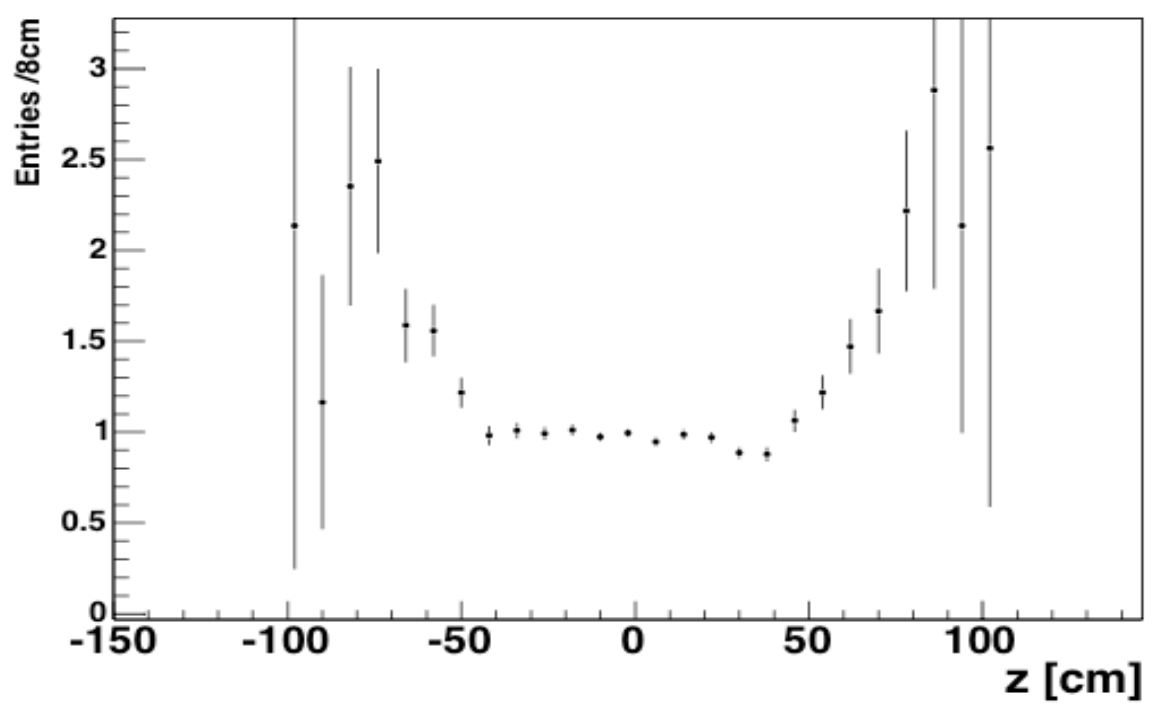

Figure 8.15: Ratio of number of events in data and PMCS, as a function of $z$. The PMCS sample is generated using a single Gaussian, with width of $26.7 \mathrm{~cm}$, and no corrections applied. The total number of events in PMCS was normalised to the total number of events in data.

The presence of backgrounds in the samples used to calculate the muon identification and trigger efficiencies gave rise to a systematic uncertainty of $0.4 \%$ [36]. Some of the trigger efficiencies are modelled in PMCS as functions of $\eta_{A}$ and $\phi_{A}$, as discussed in Section 8.2. This is done by measuring these efficiencies as one dimensional projections in $\eta_{A}$ and $\phi_{A}$ and multiplying them together to produce a two dimensional grid. To estimate a systematic uncertainty caused in this method these efficiencies are measured as single grids in $\eta_{A}$ and $\phi_{A}$ of efficiencies, which resulted in a $0.2 \%$ decrease in $\epsilon_{\mathrm{MC}}$. This difference is quoted as a systematic uncertainty.

To evaluate a systematic uncertainty caused by uncertainty on the PMCS parameters the value of $\epsilon_{\mathrm{MC}}$ was evaluated for $\mathrm{Z} / \gamma^{*} \rightarrow \mu^{+} \mu^{-}$events using samples of PMCS where the parameters had been varied. For each parameter two samples were generated; one where the value of the parameter had been increased by its uncer- 

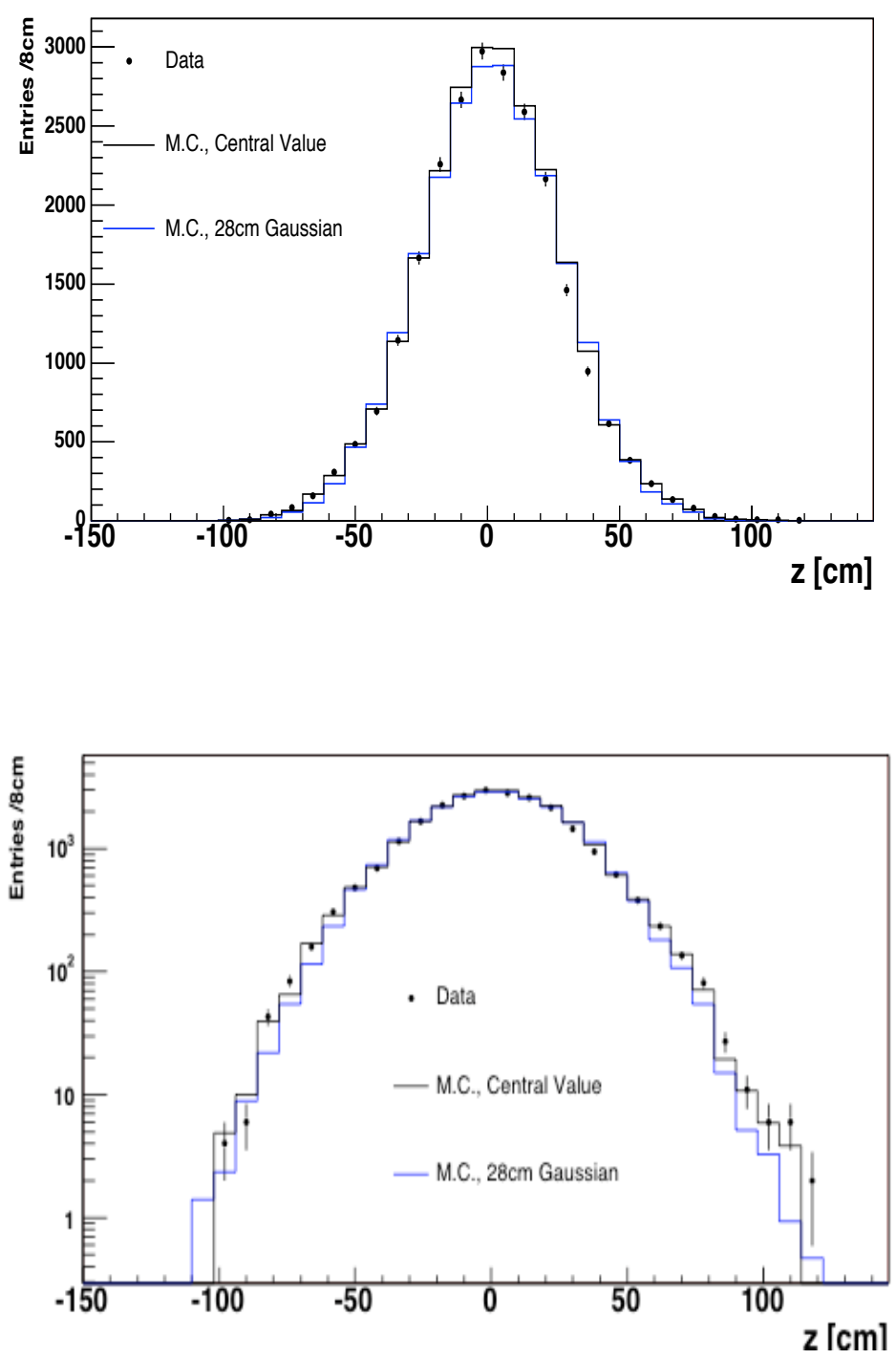

Figure 8.16: Distribution of the $z$ vertex of the tracks passing the events selection. The top plot shows this on a standard scale, the bottom on a log scale. The data is shown as the points with error bars. The PMCS predictions are shown using a black histogram for the central value and a blue histogram for the Gaussian, width $28 \mathrm{~cm}$. 


\begin{tabular}{|c|c|c|c|}
\hline \multirow[t]{2}{*}{ Systematic } & \multicolumn{3}{|c|}{ Value } \\
\hline & Period 1 & Period 2 & Period 3 \\
\hline Choice of PDF & \multicolumn{3}{|c|}{$+1.2 \% /-1.1 \%$} \\
\hline Syst-Stat & $2.5 \%$ & $0.9 \%$ & $0.6 \%$ \\
\hline PMCS/DMCS discrepancy & \multicolumn{3}{|c|}{$1.1 \%$} \\
\hline PMCS muon-ID efficiency model & \multicolumn{3}{|c|}{$1.0 \%$} \\
\hline Beamspot Parameterisation & \multicolumn{3}{|c|}{$0.6 \%$} \\
\hline Backgrounds in trigger efficiencies & \multicolumn{3}{|c|}{$0.5 \%$} \\
\hline Backgrounds in muon-ID efficiency & \multicolumn{3}{|c|}{$0.4 \%$} \\
\hline PMCS trigger efficiency models & \multicolumn{3}{|c|}{$0.2 \%$} \\
\hline PMCS parameters & \multicolumn{3}{|c|}{$0.2 \%$} \\
\hline PYTHIA parameters & \multicolumn{3}{|c|}{$0.2 \%$} \\
\hline
\end{tabular}

Table 8.4: Summary of systematic uncertainties on $\epsilon_{\mathrm{MC}}$

tainty and one where it had been decreased by its uncertainty. The only parameter which $\epsilon_{\mathrm{MC}}$ was sensitive to was the $p_{t}$ scale, which produced a change of $\pm 0.2 \%$, which is quoted as a systematic. The sensitivity of $\epsilon_{\mathrm{MC}}$ to the PYTHIA parameters used was investigated by determining $\epsilon_{\mathrm{MC}}$ with the default set of parameters, instead of the tuned set. This resulted in an increase of $\epsilon_{\mathrm{MC}}$ by $0.2 \%$, which is quoted as a systematic uncertainty.

\section{Cross-checks on $\varepsilon_{M C}$}

A series of cross-checks are included to check various aspects of the Monte Carlo simulation. The first was to evaluate the acceptance using the HERWIG generator [28]. This acceptance was compared to the acceptance using PYTHIA using the CTEQ4 PDF sets [63] and with final state photon radiation turned off, because, in the version of HERWIG used, the CTEQ4 PDF is the most recent PDF available and final 


\begin{tabular}{|l|c|c|}
\hline & HERWIG & PYTHIA \\
\hline \hline Cuts on $x_{A}$ and $y_{A}$ & $0.568 \pm 0.002$ & $0.567 \pm 0.002$ \\
Exclude calorimeter support region & $0.801 \pm 0.002$ & $0.797 \pm 0.002$ \\
Cuts on $p_{t}$ and $M_{\mu \mu}$ & $0.899 \pm 0.002$ & $0.896 \pm 0.002$ \\
\hline \hline
\end{tabular}

Table 8.5: Breakdown of the acceptance measured using HERWIG and PYTHIA. The numbers are consecutive so that the bottom hole efficiency is for those events that pass the cuts in $x_{A}$ and $y_{A}$. The cuts are defined in Section 6.1.

state photon radiation is not modelled. The geometric and kinematic acceptances are listed in Table 8.5. As can be seen they are in good agreement with each other.

The efficiencies measured in data using the tag and probe method were checked for biases using the DMCS simulation [36]. The efficiencies were measured on samples of $\mathrm{Z} / \gamma^{*} \rightarrow \mu^{+} \mu^{-}$DMCS events and measured using the tag and probe method and using the Monte Carlo truth information. Within statistical uncertainties no disagreement was found.

The trigger efficiency was compared between PMCS and an independent method. This independent method involved selecting a series of $\mathrm{Z} / \gamma^{*} \rightarrow \mu^{+} \mu^{-}$events that were identified using a series of independent, electromagnetic and jet, triggers [36]. The trigger efficiency is then the fraction of these events that meet the requirements of the muon trigger of interest.

As events with enough activity in the calorimeter to be identified by electromagnetic or jet triggers might be expected to have different kinematics, the efficiency in PMCS is only calculated using events with a jet with $E_{T}$ greater than $30 \mathrm{GeV}$ or a photon with $p_{t}$ greater than $15 \mathrm{GeV}$. Table 8.6 summarises the di-muon and single muon trigger efficiencies for the two data taking periods. As can be seen the trigger efficiency agrees between the two methods, within the statistical uncertainties.

The $\mathrm{Z} \rightarrow \mu^{+} \mu^{-}$cross section times branching ratio $\left(\sigma_{Z \rightarrow \mu^{+} \mu^{-}}\right)$was evaluated 


\begin{tabular}{|l|c|c|c|}
\hline Trigger & Period & Independent Trigger Method & PMCS \\
\hline \hline Di-muon trigger & 1 & $0.67 \pm 0.03$ & 0.67 \\
Di-muon trigger & 3 & $0.75 \pm 0.02$ & 0.73 \\
Single muon trigger & 3 & $0.86 \pm 0.01$ & 0.85 \\
\hline \hline
\end{tabular}

Table 8.6: Trigger efficiencies measured using the independent trigger method and using PMCS.

separately using different regions of the muon system. The only two quantities that are assumed to change are $\epsilon_{\mathrm{MC}}$ and the number of candidates.

- The value of $\sigma_{Z \rightarrow \mu^{+} \mu^{-}}$using only events where both muons lay in the WAMUS was determined to be $287.9 \pm 4.3$ (stat) $\mathrm{pb}^{-1}$.

- The value of $\sigma_{Z \rightarrow \mu^{+} \mu^{-}}$using only events where both muons lay in the FAMUS was determined to be $286.3 \pm 7.5$ (stat) $\mathrm{pb}^{-1}$.

- The value of $\sigma_{Z \rightarrow \mu^{+} \mu^{-}}$using only events where one muon lay in the WAMUS and the other in the FAMUS was determined to be $292.7 \pm 4.1$ (stat) $\mathrm{pb}^{-1}$.

As can be seen all three agree within the statistical uncertainty. The final cross check was to vary the mass cut, from $40 \mathrm{GeV}$ to $60 \mathrm{GeV}$, and see how the $\mathrm{Z} \rightarrow \mu^{+} \mu^{-}$ cross section varies. This led to changes in the number of candidate events, in $\epsilon_{\mathrm{MC}}$ and in the fraction of events attributed to background events. In addition the theoretical correction factor used to obtain the $\mathrm{Z} \rightarrow \mu^{+} \mu^{-}$cross section from the $\mathrm{Z} / \gamma^{*} \rightarrow \mu^{+} \mu^{-}$cross section, as described in Section 9.1, changes.

The cross section changes by $0.4 \%$ and the statistical uncertainty on the change in the candidate events is $0.3 \%$. As there are other sources of uncorrelated uncertainty this was assumed to be in agreement. 


\subsubsection{Other Analyses using PMCS}

These three analyses all use some of the authors work on the PMCS simulation and are chosen to demonstrate the wide use and good performance of the simulation. They also happen to illustrate three main types of studies that are performed with electroweak bosons.

\section{Study of Production of Electroweak Bosons in Association with Photons.}

The first of the other analyses discussed is the study of events where an electroweak boson is produced with a high $E_{T}$ photon $[64,65]$. This measurement of these cross sections is another test of Standard Model predictions. In addition the study of the kinematics of these events can be used to measure the coupling between the electroweak bosons and the photon, providing a further probe of the Standard Model.

These measurements used the PMCS simulation to generate a series of events to calculate the acceptances. Figure 8.3.2 shows a comparison of the transverse mass of the $W \gamma$ system between the data and the PMCs Monte Carlo simulation. This figure includes data from both the muon and electron channels.

\section{Measurement of the Diffractive $Z \rightarrow \mu^{+} \mu^{-}$Cross Section}

The second analysis discussed is a measurement of the diffractive $\mathrm{Z} / \gamma^{*} \rightarrow \mu^{+} \mu^{-}$ cross section. This measurement starts off from the sample of $\mathrm{Z} / \gamma^{*} \rightarrow \mu^{+} \mu^{-}$events selected in Section 6.1.1 and looks what fraction of these can be attributed to diffractive production [66]. Diffractive events are where one or both of the incoming hadrons remain intact after the hard scatter. Events of $\mathrm{Z} / \gamma^{*} \rightarrow \mu^{+} \mu^{-}$are used as they provide a clean tag, with little activity in the calorimeter, to study diffractive processes. 


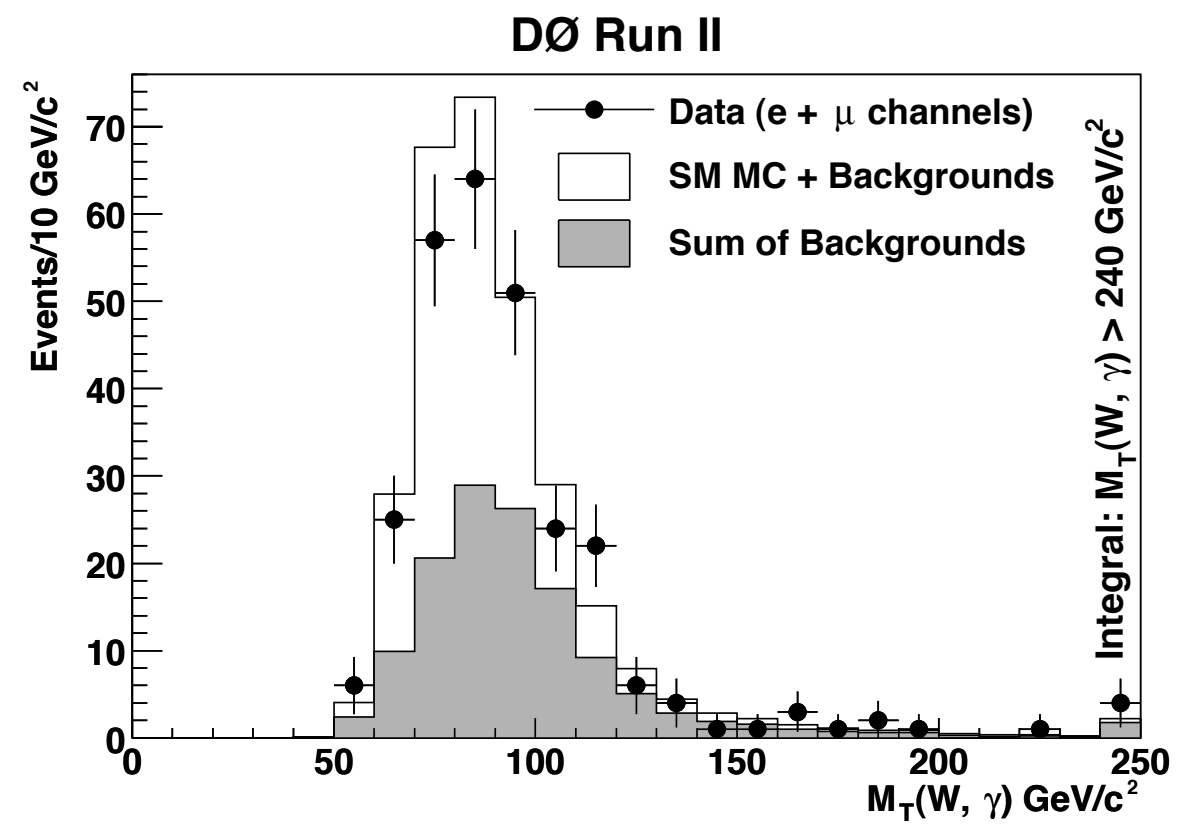

Figure 8.17: Comparison between of the transverse mass distribution of $W \gamma$ pairs in data (points) and PMCS(black line). The contribution from background processes is represented by the grey histogram [64]

The efficiency of this analysis was determined in the same way that $\epsilon_{\mathrm{MC}}$ was calculated in Section 8.3.1. The only difference was that this analysis used the diffractive Monte Carlo generator, POMWIG [67], to generate events instead of PYTHIA. Comparisons between distributions in the Monte Carlo samples and data were made, such as that shown in Figure 8.18.

\section{Search for Large Extra Spatial Dimensions in Di-muon Production}

The final analysis is one of many that look for BSM physics by looking for new resonant states. This analysis looks for the cases where these resonances decay in the di-muon channel [68]. These resonant states are associated with gravitons propagating through new extra dimensions. The model reduces the Planck scale to 


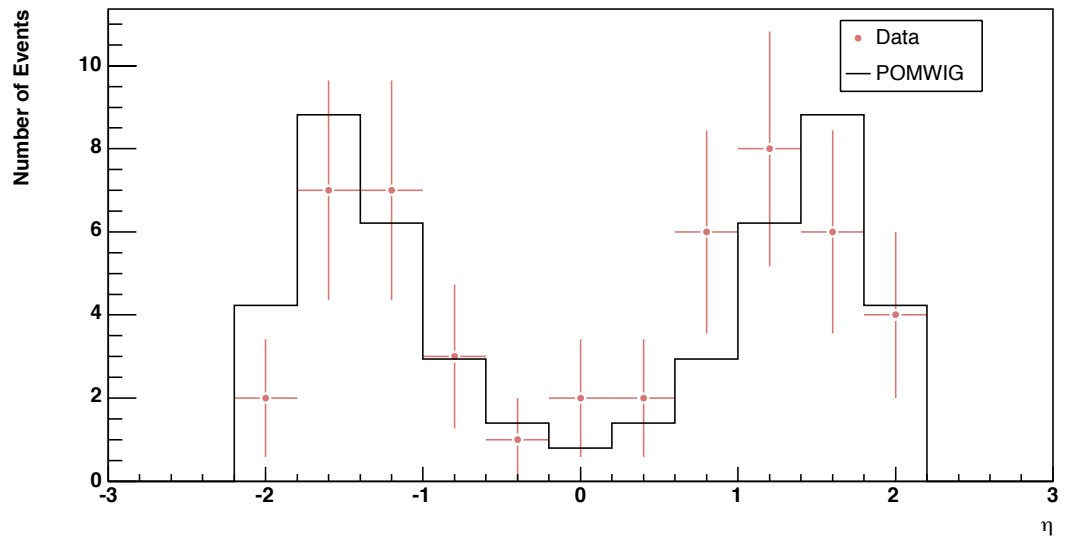

Figure 8.18: Comparison of $\eta$ distribution in data (red points) and PMCS (black line) for events attributed to diffractive $\mathrm{Z} \rightarrow \mu^{+} \mu^{-}$production.

much lower energies, which can provide an explanation of such features of the Standard Model as the hierarchy problem. The analysis uses the $\mathrm{Z} / \gamma^{*} \rightarrow \mu^{+} \mu^{-}$peak as a calibration tool, tuning the parameterisation and efficiencies. The parameterisation used is that described in Section 4.3.1, although some of the parameters are retuned to reflect the different track quality requirements in this analysis. 


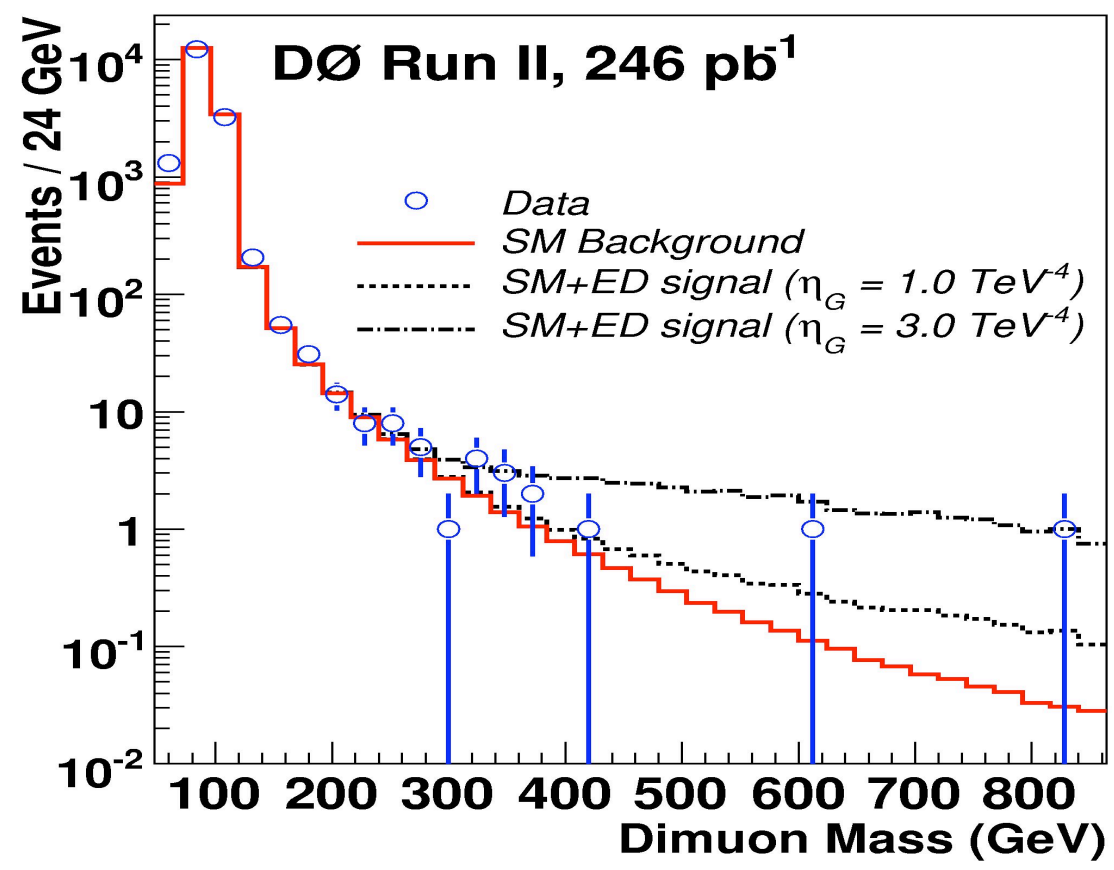

Figure 8.19: Comparison between data and parameterised Monte Carlo. The blue circles are data, the red line is $\mathrm{Z} / \gamma^{*} \rightarrow \mu^{+} \mu^{-}$Monte Carlo. The signal expected to be observed for different Planck scales are shown [68]. 


\section{Chapter 9}

\section{Indirect Measurement of the Width of the W Boson}

To calculate the width of the $\mathrm{W}$ boson using Equation 5.8 the ratio of the $\mathrm{W} \rightarrow \mu \nu$ to $\mathrm{Z} \rightarrow \mu^{+} \mu^{-}$cross sections, $R$, needs to be obtained from the results given in Section 6.5. The first step is to obtain the $\mathrm{Z} \rightarrow \mu^{+} \mu^{-}$cross section from the $\mathrm{Z} / \gamma^{*} \rightarrow \mu^{+} \mu^{-}$cross section. This is done using a theoretical relationship, known as the Drell-Yan correction, as described in Section 9.1. Then the results are combined to calculate $R$ in Section 9.2. In Section 9.3 the $\mathrm{W}$ boson width is obtained from $R$ and compared to other results in Section 9.4.

\subsection{The Drell-Yan Correction}

This section describes the theoretical correction used to obtain the $\mathrm{Z} \rightarrow \mu^{+} \mu^{-}$cross section from the $\mathrm{Z} / \gamma^{*} \rightarrow \mu^{+} \mu^{-}$cross section. The correction factor was derived to replace the previous form which is included for comparison in Appendix C. The new method is more efficient, removing the need to generate any Monte Carlo events. 


\subsubsection{Derivation of the Drell-Yan Correction.}

The Drell-Yan correction assumes the following relationship:

$$
\frac{\sigma_{\mathrm{Z} \rightarrow \mu^{+} \mu^{-}}}{\sigma_{\mathrm{Z} / \gamma^{*} \rightarrow \mu^{+} \mu^{-}}}(\text {theory })=\frac{\sigma_{\mathrm{Z} \rightarrow \mu^{+} \mu^{-}}}{\sigma_{\mathrm{Z} / \gamma^{*} \rightarrow \mu^{+} \mu^{-}}}(\operatorname{expt})
$$

where the left hand side of equation 9.1 are theoretical predictions of the $\mathrm{Z} \rightarrow \mu^{+} \mu^{-}$ and $\mathrm{Z} / \gamma^{*} \rightarrow \mu^{+} \mu^{-}$cross sections. On the right hand side is the $\mathrm{Z} / \gamma^{*} \rightarrow \mu^{+} \mu^{-}$cross section, as measured in Chapter 6 , and the unknown $\mathrm{Z} \rightarrow \mu^{+} \mu^{-}$cross section. Equation 9.1 can be rearranged to

$$
\sigma_{\mathrm{Z} \rightarrow \mu^{+} \mu^{-}}(\text {expt })=\frac{\sigma_{\mathrm{Z}} \rightarrow \mu^{+} \mu^{-}}{\sigma_{\mathrm{Z} / \gamma^{*} \rightarrow \mu^{+} \mu^{-}}}(\text {theory }) \sigma_{\mathrm{Z} / \gamma^{*} \rightarrow \mu^{+} \mu^{-}}(\text {expt })
$$

This can be expressed as

$$
\sigma_{\mathrm{Z} \rightarrow \mu^{+} \mu^{-}}(\operatorname{expt})=R_{\sigma} \sigma_{\mathrm{Z} / \gamma^{*} \rightarrow \mu^{+} \mu^{-}}(\operatorname{expt})
$$

where $R_{\sigma}$ is the Drell-Yan correction, defined as

$$
R_{\sigma} \equiv \frac{\sigma_{\mathrm{Z} \rightarrow \mu^{+} \mu^{-}}}{\sigma_{\mathrm{Z} / \gamma^{*} \rightarrow \mu^{+} \mu^{-}}}(\text {theory })
$$

The values of these cross sections can be calculated using Monte Carlo programs such as PYTHIA [27] or MC@NLO [29].

\subsubsection{Calculation of the Drell Yan Correction}

The MC@NLO program is chosen to evaluate $R_{\sigma}$ using the CTEQ6.1 PDF sets [54]. As a cross check the value is also calculated using PYTHIA. The values of the $\mathrm{Z} \rightarrow \mu^{+} \mu^{-}$ 


\begin{tabular}{|l|c|c|}
\hline & PYTHIA & MC@NLO \\
\hline$\sigma_{\mathrm{Z} \rightarrow \mu^{+} \mu^{-}[\mathrm{pb}]}$ & $197.6 \pm 0.3$ & 236.4 \\
$\sigma_{\mathrm{Z} / \gamma^{*} \rightarrow \mu^{+} \mu^{-}[\mathrm{pb}]}$ & $222.9 \pm 0.3$ & 267.2 \\
\hline$R_{\sigma}$ & $0.886 \pm 0.002$ & 0.885 \\
\hline \hline
\end{tabular}

Table 9.1: Values of the cross sections used to calculate the Drell Yan correction.

and $\mathrm{Z} / \gamma^{*} \rightarrow \mu^{+} \mu^{-}$cross sections are summarised in table 9.1. The uncertainty on the PYTHIA cross sections is due to statistical limitations. The statistical uncertainty on the MC@NLO figures is negligible.

The difference between the cross sections arise from the fact that PYTHIA is a LO generator as opposed to MC@NLO which is a NLO generator. However the fact that the Drell-Yan correction is the same for the two generators would indicates that it is insensitive to whether it is calculated to LO or NLO. The uncertainty due to choice of PDF is evaluated, using the prescriptions described in Section 7, to be $\pm 0.2 \%$.

\subsection{Measurement of $R$}

This section describes the measurement of the ratio of the $\mathrm{W} \rightarrow \mu \nu$ to $\mathrm{Z} \rightarrow \mu^{+} \mu^{-}$ cross sections, $R$. The $\mathrm{Z} / \gamma^{*} \rightarrow \mu^{+} \mu^{-}$and $\mathrm{W} \rightarrow \mu \nu$ cross sections are each evaluated using Equation 6.1. Only the third data taking period in the $\mathrm{Z} / \gamma^{*} \rightarrow \mu^{+} \mu^{-}$ analysis is used, which corresponds to the same data taking period as used by the $\mathrm{W} \rightarrow \mu \nu$ analysis. Restricting the data set used by the $\mathrm{Z} / \gamma^{*} \rightarrow \mu^{+} \mu^{-}$analysis leads to a negligible increase in the overall uncertainty, but makes the evaluation of the correlations between the $\mathrm{Z} / \gamma^{*} \rightarrow \mu^{+} \mu^{-}$and $\mathrm{W} \rightarrow \mu \nu$ cross sections much simpler. The $\mathrm{Z} \rightarrow \mu^{+} \mu^{-}$cross section is obtained from the $\mathrm{Z} / \gamma^{*} \rightarrow \mu^{+} \mu^{-}$cross section using the Drell Yan correction as described in Section 9.1.

Both trigger configurations from the $\mathrm{W} \rightarrow \mu \nu$ analysis are used. The $\mathrm{Z} / \gamma^{*} \rightarrow \mu^{+} \mu^{-}$ 
analysis only used the 'wide' configuration of the single muon trigger, which was always available, even during the periods when the $\mathrm{W} \rightarrow \mu \nu$ analysis used the 'all' configuration. The $\mathrm{W} \rightarrow \mu \nu$ cross section is evaluated separately for these two configurations and the $\mathrm{Z} / \gamma^{*} \rightarrow \mu^{+} \mu^{-}$is evaluated once, with all quantities averaged over the two configurations. The value of $R$ is determined separately for the two configurations, using the separate $\mathrm{W} \rightarrow \mu \nu$ cross sections and the averaged $\mathrm{Z} / \gamma^{*} \rightarrow \mu^{+} \mu^{-}$ cross section. Averaging the $\mathrm{Z} / \gamma^{*} \rightarrow \mu^{+} \mu^{-}$cross section over the two configurations is acceptable because the $\mathrm{Z} / \gamma^{*} \rightarrow \mu^{+} \mu^{-}$cross section exhibits no time dependence.

Section 9.2.1 describes an evaluation of the correlations between the $\mathrm{W} \rightarrow \mu \nu$ and $\mathrm{Z} / \gamma^{*} \rightarrow \mu^{+} \mu^{-}$cross sections. After these correlations have been accounted for the ratio itself is calculated in Section 9.2.2. This section describes the evaluation of the ratio for the two different trigger configurations and the combination of these two results into the final one.

\subsubsection{Correlated Uncertainties}

This section describes the evaluation of correlations between the systematic uncertainties in the $\mathrm{W} \rightarrow \mu \nu$ and $\mathrm{Z} \rightarrow \mu^{+} \mu^{-}$analyses. The only quantities that need evaluating are the efficiencies of the event selection because the systematic uncertainties on the integrated luminosity are completely correlated and the uncertainties on the background fractions and number of candidate events are completely uncorrelated.

A detailed description of all the uncertainties on $\epsilon_{\mathrm{MC}}$ for the $\mathrm{Z} / \gamma^{*} \rightarrow \mu^{+} \mu^{-}$analysis is given in Section 8.3.1. A detailed description of most of the uncertainties on $\epsilon_{\mathrm{MC}}$ for the $\mathrm{W} \rightarrow \mu \nu$ analysis is given in [42], although a few new sources of systematic are evaluated for consistency with the $\mathrm{Z} / \gamma^{*} \rightarrow \mu^{+} \mu^{-}$analysis. The approach taken to evaluating the correlations is to examine every systematic uncertainty on both acceptances and determine the degree of correlation, commencing with that 


\begin{tabular}{|l|c|c|c|}
\hline & 'All' & 'Wide' & Covariance \\
\hline \hline TevEWWG & $+0.7 \% /-1.4 \%$ & $+0.8 \% /-1.3 \%$ & $+0.7 \% /-1.3 \%$ \\
DØ & $+0.7 \% /-1.5 \%$ & $+0.8 \% /-1.4 \%$ & $+0.7 \% /-1.4 \%$ \\
'old' CTEQ & $\pm 0.8 \%$ & $\pm 0.9 \%$ & $\pm 0.8 \%$ \\
\hline
\end{tabular}

Table 9.2: Summary of PDF Uncertainties using three different prescriptions.

due to choice of PDF.

\section{The PDF Uncertainty}

The PDF Uncertainty is evaluated using the CTEQ 6.1 PDF sets and the prescriptions derived in Section 7. All the quantities used in evaluating $R$ that are sensitive to the choice of PDF are combined to produce an observable, $X$ :

$$
X \equiv \frac{R_{\sigma} \varepsilon_{W \rightarrow \mu \nu}}{\varepsilon_{Z \rightarrow \mu^{+} \mu^{-}}} .
$$

The fractional uncertainty due to choice of PDF on $R$ is equal to the fractional uncertainty due to choice of PDF on $X$, because the other quantities used to evaluate $R$ are insensitive to the choice of PDF.

The procedure adopted was to generate 92 million events for both the $\mathrm{W} \rightarrow \mu \nu$ and $\mathrm{Z} / \gamma^{*} \rightarrow \mu^{+} \mu^{-}$analyses; 12 million of these for the central value and 2 million for each of the error PDFs. These events were passed through the PMCs simulation and used to determine the acceptances for the $\mathrm{W} \rightarrow \mu \nu$ and $\mathrm{Z} / \gamma^{*} \rightarrow \mu^{+} \mu^{-}$analyses. The uncertainty was then determined using the three prescriptions described in Section 7. These are summarised in Table 9.2. As expected, the old CTEQ prescription underestimates the uncertainty. The D $\varnothing$ and TeVEWWG prescriptions give very similar answers. Both are quoted here, though the answer that gives the largest uncertainty is chosen. 
The central value is evaluated using the CTEQ6.1 NLO PDF sets and the PYTHIA generator. The acceptances are also evaluated using the CTEQ6 LO PDF sets and the difference quoted as a systematic uncertainty. This leads to a systematic uncertainty of $1.0 \%$ for the 'wide' data taking period and $1.8 \%$ for the 'all' data taking period. This uncertainty can also be thought of as an indication of a source of systematic uncertainty due to theoretical errors in the PDF fitting which are not fully covered by the PDF uncertainty above. All other sources of theoretical uncertainty, such as that on $\alpha_{s}$, were treated as being negligible.

\section{The Syst-stat Uncertainty}

The syst-stat uncertainty was evaluated using the technique described in Section 8.2.4. It was determined for the $\mathrm{W} \rightarrow \mu \nu$ and $\mathrm{Z} / \gamma^{*} \rightarrow \mu^{+} \mu^{-}$analyses to be $0.9 \%$ and $0.6 \%$ respectively. Adding these two uncertainties in quadrature yields a syst-stat uncertainty of $1.1 \%$. The spread of the value of $R$ when evaluating the uncertainty is shown in Figure 8.8. This ignores the fact that the syst-stat uncertainty on the $\mathrm{Z} / \gamma^{*} \rightarrow \mu^{+} \mu^{-}$and $\mathrm{W} \rightarrow \mu \nu$ are correlated.

This correlation results from the use of the same set of $\mathrm{Z} / \gamma^{*} \rightarrow \mu^{+} \mu^{-}$events to determine the tracking and muon identification efficiencies. The tighter quality requirements in the $\mathrm{W} \rightarrow \mu \nu$ analysis made on tracks, and muons results in efficiencies that differ between the two analyses. To evaluate the correlations between the two analyses the tracking and muon identification efficiencies in the $\mathrm{W} \rightarrow \mu \nu$ analysis were each replaced by two efficiencies. In the $\mathrm{W} \rightarrow \mu \nu$ analysis the tracking efficiency was replaced by the efficiency for a track to be reconstructed, i.e. the tracking efficiency as used by the $\mathrm{Z} / \gamma^{*} \rightarrow \mu^{+} \mu^{-}$analysis, and then the efficiency for a reconstructed track to meet the track quality requirements used by the $\mathrm{W} \rightarrow \mu \nu$ analysis. Similarly the efficiency for a muon to pass the medium quality cuts was replaced by the efficiency for a muon to pass the loose quality cuts and the probability for a muon passing the loose quality cuts to pass the medium quality cuts. 


\begin{tabular}{|l|c|c|c|}
\hline & 'All' & 'Wide' & Covariance \\
\hline \hline Correlations modelled & $1.1 \%$ & $1.2 \%$ & $0.6 \%$ \\
Correlations ignored & $1.1 \%$ & $1.2 \%$ & $0.6 \%$ \\
\hline
\end{tabular}

Table 9.3: Summary of syst-stat Uncertainties with correlations properly accounted for and ignored.

No attempt was made to model the correlations in the trigger efficiencies, due to the complexity of the model used. This leads to a small overestimation of the overall uncertainty. The effect cannot be large as the syst-stat uncertainty in the $\mathrm{Z} / \gamma^{*} \rightarrow \mu^{+} \mu^{-}$analysis is dominated by the tracking and muon identification efficiencies.

The syst-stat uncertainty was then recalculated by fluctuating the tracking and loose muon efficiencies coherently in the two analyses. The results are summarised in Table 9.3. The two different rows show the systematic for the case where the correlations are modelled and the case where the correlations are ignored. The uncertainty on $R$ is the same for both methods, which is not what would be naively expected. This is due to the fact that the data samples used to measure the efficiencies for the $\mathrm{W} \rightarrow \mu \nu$ and $\mathrm{Z} \rightarrow \mu^{+} \mu^{-}$analyses are slightly different, due to the tighter data quality requirements made by the $\mathrm{W} \rightarrow \mu \nu$ analysis. This leads to statistical fluctuations diluting some of the correlations.

\section{PMCS-DMCS Discrepancy}

In the $\mathrm{Z} / \gamma^{*} \rightarrow \mu^{+} \mu^{-}$analysis the value of the efficiency of the selection cuts was evaluated using the DMCS simulation. The difference between the efficiency as evaluated using DMCS and using PMCS is quoted as a systematic, as described in Section 8.3.1. No such cross-check was made in the $\mathrm{W} \rightarrow \mu \nu$ analysis. The fractional systematic uncertainty on the $\mathrm{Z} / \gamma^{*} \rightarrow \mu^{+} \mu^{-}$cross section was taken as the fractional systematic 
uncertainty on $R$. This is an overestimate as the effect of any discrepancy between the two simulations would affect both analyses in a correlated fashion.

\section{Boundary Variations}

The $\mathrm{W} \rightarrow \mu \nu$ analysis was found to be insensitive to the definition of the octant boundaries, even when the loose efficiency is used. Therefore the systematic uncertainty caused by the definitions of the octant boundaries is the same as the systematic uncertainty on the $\mathrm{Z} / \gamma^{*} \rightarrow \mu^{+} \mu^{-}$analysis, namely $1.0 \%$.

\section{Backgrounds in the Efficiency Samples}

In the $\mathrm{Z} / \gamma^{*} \rightarrow \mu^{+} \mu^{-}$analysis a systematic uncertainty was calculated to account for backgrounds in the data samples used to determine the muon identification, tracking and triggering efficiencies. No such assessment was made in the $\mathrm{W} \rightarrow \mu \nu$ analysis. The fractional systematic uncertainty on the $\mathrm{Z} / \gamma^{*} \rightarrow \mu^{+} \mu^{-}$cross section was taken as the fractional systematic uncertainty on $R$. This is probably an overestimate as the effect of backgrounds in the efficiency samples are correlated leading to cancellation on the ratio.

\section{PMCS parameterisation}

There are several uncertainties caused by the parameterisation of the detector simulation. Some of these, such as the $E_{T}$ parameterisation, leading to an uncertainty of $0.6 \%$, are restricted to only the $\mathrm{W} \rightarrow \mu \nu$ analyses. Others such as the $p_{t}$ tuning and the tuning of the vertex distribution affect both analyses.

The largest uncertainty in both analyses caused by the PMCS parameterisation was due to the modelling of the $z$ distribution of the beamspot. Both analyses used the same model for the beamspot distribution, a Gaussian of width $26.7 \mathrm{~cm}$, 
which was obtained by tuning to $\mathrm{W} \rightarrow \mu \nu$ data. However the two analyses adopted different approaches to evaluating systematic uncertainties.

The $\mathrm{W} \rightarrow \mu \nu$ analysis reevaluated the acceptance using the upper and lower bounds of the beamspot tune [42] to produce a change in the acceptance of $0.6 \%$ in the 'all' region and $0.8 \%$ in the 'wide' region. When this approach is used for the $\mathrm{Z} / \gamma^{*} \rightarrow \mu^{+} \mu^{-}$analysis the resulting uncertainty is $0.2 \%$. The $\mathrm{Z} / \gamma^{*} \rightarrow \mu^{+} \mu^{-}$ analysis adopted another, more conservative approach, as described in Section 8.3.1 resulting in an uncertainty of $0.6 \%$. The approach taken in the ratio is to treat $0.2 \%$ as being correlated between the two analyses and retain the rest, resulting in an error of $0.8 \%$ in the all region and $1.0 \%$ in the wide region.

The only other component of the parameterisation that could be correlated is the $p_{t}$ tuning. It was difficult to assess what fraction is correlated and as the systematic was only small the uncertainties are treated as being uncorrelated.

\section{Choice of PYTHIA parameters.}

An estimate of the sensitivity of $R$ to the choice of PYTHIA parameters was made by re-evaluating the acceptances using the default set of PYTHIA parameters. This resulted in a $0.2 \%$ increase in the $\mathrm{Z} / \gamma^{*} \rightarrow \mu^{+} \mu^{-}$acceptance and a $0.4 \%$ increase in the $\mathrm{W} \rightarrow \mu \nu$ acceptances. This results in a $0.2 \%$ decrease in $R$, which is quoted as a systematic uncertainty.

\section{Other Systematic Uncertainties}

This section lists the remaining systematic uncertainties on the efficiencies.

- Time Variation of the Efficiencies

Only the $\mathrm{W} \rightarrow \mu \nu$ analysis was found to be sensitive to time dependence of the 
efficiencies, due to the requirement of hits in the SMT [42]. The Z/ $\gamma^{*} \rightarrow \mu^{+} \mu^{-}$ analysis was tested for time dependence but found to be insensitive [36].

- Cosmic and Isolation Cut Efficiencies

Both analyses have uncertainties assigned to both these efficiencies, but they can both be taken as completely uncorrelated. This is because the manner in which these cuts are applied and the method by which their efficiencies are determined are different.

- Opposite Sign Charge Cut

As this cut is only present in the $\mathrm{Z} / \gamma^{*} \rightarrow \mu^{+} \mu^{-}$analysis, there is no correlation in this systematic between the analyses.

\subsubsection{Determination of $R$.}

In this section the measurements of the $\mathrm{Z} / \gamma^{*} \rightarrow \mu^{+} \mu^{-}$and $\mathrm{W} \rightarrow \mu \nu$ cross sections are combined to determine $R$. The values of $R$ for the two trigger configurations are combined to produce the final value which is then used to determine the $\mathrm{W}$ width. Table 9.4 lists all the quantities used to determine $R$ for the two data taking periods. It concludes with a calculation of $R$ for the two trigger configurations.

These two values of $R$ are then combined according to their uncorrelated uncertainties. To do this all the uncertainties are summed up in Tables 9.5 to 9.7, noting in particular the covariance between the two different trigger configurations. They are split into different tables for uncertainty caused by limited statistics in the number of candidate events (Table 9.5), the uncertainty caused by uncertainties on the backgrounds (Table 9.6) and the uncertainty caused by uncertainties on the efficiencies (Table 9.7). The analysis is dominated by systematic uncertainties, particularly by those on the efficiencies.

Combining all the sources of uncertainty $R$ is determined to be 


\begin{tabular}{|l|c|c|}
\hline & 'All' & 'Wide' \\
\hline \hline$R_{\sigma}$ & \multicolumn{2}{|c|}{0.885} \\
\hline$N_{e v}\left(\mathrm{Z} / \gamma^{*} \rightarrow \mu^{+} \mu^{-}\right)$ & \multicolumn{2}{|c|}{11556} \\
$C_{b k}\left(\mathrm{Z} / \gamma^{*} \rightarrow \mu^{+} \mu^{-}\right)$ & \multicolumn{2}{|c|}{0.986} \\
$\varepsilon\left(\mathrm{Z} / \gamma^{*} \rightarrow \mu^{+} \mu^{-}\right)$ & \multicolumn{2}{|c|}{0.317} \\
$\int \mathcal{L} \mathrm{d} t\left(\mathrm{Z} / \gamma^{*} \rightarrow \mu^{+} \mu^{-}\right)\left(p b^{-1}\right)$ & \multicolumn{2}{|c|}{109.4} \\
\hline$N_{e v}(\mathrm{~W} \rightarrow \mu \nu)$ & 29159 & 33126 \\
$C_{b k}(\mathrm{~W} \rightarrow \mu \nu)$ & 0.918 & 0.926 \\
$\varepsilon(\mathrm{W} \rightarrow \mu \nu)$ & 0.211 & 0.186 \\
$\int \mathcal{L} \mathrm{d} t(\mathrm{~W} \rightarrow \mu \nu)\left(p b^{-1}\right)$ & 41.9 & 53.7 \\
\hline$R$ & 10.41 & 10.56 \\
\hline
\end{tabular}

Table 9.4: Summary of the numbers used to calculate $R$.

\begin{tabular}{|l|c|c|c|}
\hline & 'All' & 'Wide' & Covariance \\
\hline \hline $\mathrm{Z} / \gamma^{*} \rightarrow \mu^{+} \mu^{-}$ & $0.9 \%$ & $0.9 \%$ & $0.9 \%$ \\
$\mathrm{~W} \rightarrow \mu \nu$ & $0.6 \%$ & $0.6 \%$ & 0 \\
\hline
\end{tabular}

Table 9.5: Summary of the uncertainties due to the statistical limitations on the number of candidate events.

$$
R=10.50 \pm 0.11(\text { stat }) \pm 0.30(\text { syst })_{-0.15}^{+0.08}(\mathrm{pdf})
$$

\subsection{Width of the W Boson from $R$}

Rearranging Equation 5.8 the width of the $\mathrm{W}$ boson can be obtained using

$$
\Gamma_{W}=\frac{1}{R} \frac{\sigma_{W}}{\sigma_{Z}}\left(\frac{\Gamma_{Z}}{\Gamma_{\mathrm{Z} \rightarrow \mu^{+} \mu^{-}}}\right) \Gamma_{\mathrm{W}} \rightarrow \mu \nu
$$




\begin{tabular}{|l|c|c|c|}
\hline & 'All' & 'Wide' & Covariance \\
\hline \hline QCD Backgrounds & $0.8 \%$ & $0.8 \%$ & $0.8 \%$ \\
Electroweak Backgrounds & $0.4 \%$ & $0.4 \%$ & $0.4 \%$ \\
Cosmic Backgrounds & $0.2 \%$ & $0.2 \%$ & $0.2 \%$ \\
\hline
\end{tabular}

Table 9.6: Summary of the uncertainties on the backgrounds.

\begin{tabular}{|l|c|c|c|}
\hline & 'All' & 'Wide' & Correlated \\
\hline \hline PDF & $+0.7 \% /-1.5 \%$ & $+0.8 \% /-1.4 \%$ & $+0.7 \% /-1.4 \%$ \\
PDF (theory) & $1.8 \%$ & $1.0 \%$ & $1.0 \%$ \\
PMCS-DMCS Discrepancy & $1.1 \%$ & $1.1 \%$ & $1.1 \%$ \\
Syst-Stat & $1.2 \%$ & $1.2 \%$ & $0.6 \%$ \\
Octant Boundaries & $1.0 \%$ & $1.0 \%$ & $1.0 \%$ \\
PMCS parameterisation & $1.0 \%$ & $1.2 \%$ & $1.0 \%$ \\
Backgrounds in Efficiency Samples & $0.6 \%$ & $0.6 \%$ & $0.6 \%$ \\
Modelling of ISR and FSR & & & \\
Isolation Efficiencies & $0.9 \%$ & $0.9 \%$ & $0.1 \%$ \\
Cosmic Cuts (Z/ $\left.\gamma^{*} \rightarrow \mu^{+} \mu^{-}\right)$ & $0.6 \%$ & $0.6 \%$ & $0.6 \%$ \\
Opposite Sign Efficiency $\left(\mathrm{Z} / \gamma^{*} \rightarrow \mu^{+} \mu^{-}\right)$ & $0.1 \%$ & $0.1 \%$ & $0.1 \%$ \\
Modelling of Veto & $1.5 \%$ & $1.5 \%$ & $1.5 \%$ \\
Time Variation of Efficiencies & $0.6 \%$ & $0.6 \%$ & $0.6 \%$ \\
Biases in efficiency measurements & $0.4 \%$ & $0.4 \%$ & $0.4 \%$ \\
\hline
\end{tabular}

Table 9.7: Summary of the uncertainties due to uncertainties on the acceptances. 
The ratio, $\sigma_{W} / \sigma_{Z}$, is calculated at NNLO level using the CTEQ 6.1 PDF sets, to be $3.369 \pm 0.003$ (scale) $\pm 0.014(\mathrm{PDF})[55,56,69]$. This is in good agreement to the value used in [70] which calculates $\sigma_{W} / \sigma_{Z}=3.361 \pm 0.016 \mathrm{GeV}$.

The ratio, $\Gamma_{Z} / \Gamma_{Z \rightarrow \mu^{+} \mu^{-}}$, is taken from experimental measurements made at LEP to be $0.033658 \pm 0.000023[2]$. The partial width, $\Gamma_{\mathrm{W}} \rightarrow \mu \nu$, is measured to be $226.4 \pm 0.3 \mathrm{MeV}[2]$.

These values are combined with the value of $R$ as measured in Section 9.2. The uncertainty due to choice of PDF is reevaluated, to account for correlations between $R$ and $\sigma_{W} / \sigma_{Z}$, to be $+0.8 \% /-1.2 \%^{1}$. Thus the width of the $\mathrm{W}$ boson is determined to be

$$
\Gamma_{W}=2168 \pm 22(\text { stat }) \pm 62(\text { syst })_{-16}^{+24}(\text { pdf }) \pm 4(\text { other }) \mathrm{MeV}
$$

where the stat uncertainty is due to limited statistics in the number of $\mathrm{Z} / \gamma^{*} \rightarrow \mu^{+} \mu^{-}$ and $\mathrm{W} \rightarrow \mu \nu$ candidates. The syst uncertainty is a summary of most systematic uncertainties. The remaining uncertainties are due to theoretical factors; the PDF uncertainty is due to the choice of PDF and other covers theoretical uncertainties in the extraction of the $\mathrm{W}$ width from $R$.

\subsection{Discussion}

The Standard Model prediction for the width of the $\mathrm{W}$ boson is $\Gamma_{W}=2.0921 \pm$ $0.0025 \mathrm{GeV}[2]$. The Current World average of experimental measurements of the $\mathrm{W}$ width is $\Gamma_{W}=2.138 \pm 0.044 \mathrm{GeV}$ [2]. This world average includes indirect measurements of the width, from UA1, UA2, CDF Run I and D $\varnothing$ Run I, and direct

\footnotetext{
${ }^{1}$ This is evaluated using the $\mathrm{D} \varnothing$ prescription which provides slightly larger $(0.1 \%)$ uncertainties than the TevEWWG prescription.
} 
measurements of the width from CDF Run I, Dø Run I, Aleph, Delphi, L3 and Opal [2].

Since the compilation of this world average, further measurements of the $\mathrm{W}$ width have been made by DØ and CDF Run II and LEP have updated their measurement. As well as this measurement D $\varnothing$ has measured $R$ in the electron channel. Using an integrated luminosity of $177 \mathrm{pb}^{-1}$, the width of the $\mathrm{W}$ Boson was found to be $\Gamma_{W}$ $=2.080+0.054 /-0.052 \mathrm{GeV}[71]$.

CDF has also measured the width, using this indirect method. The first measurement is performed with $72 \mathrm{pb}^{-1}$ of data using both the muon and electron channels. This determined that the width was $\Gamma_{W}=2.079 \pm 0.041 \mathrm{GeV}$ [72]. This measurement has been updated in the muon channel with $200 \mathrm{pb}^{-1}$ providing a width of $\Gamma_{W}=2.056+0.044 /-0.048 \mathrm{GeV}$ [73]. DØ has also directly measured the width by looking at the shape of the $m_{T}$ distribution in $\mathrm{W} \rightarrow e \nu$ events. This measurement is made with the same data sample as the indirect measurement and determines the width to be $\Gamma_{W}=2.01 \pm 0.14 \mathrm{GeV}[26]$. The updated LEP result is $\Gamma_{W}=2.196 \pm 0.083 \mathrm{GeV}[74]$, although this is highly correlated with the previous LEP result used in the PDG average.

Figure 9.1 displays the result obtained in this thesis and compares it to the Standard Model prediction and the other experimental measurements. The experimental results are displayed as points with error bars and the Standard Model prediction is displayed as a solid line.

It can be observed that the measurement is in good agreement with both the Standard Model prediction and other experimental results. The uncertainty is slightly larger than the other results from the Tevatron using the ratio method. This is a result of the smaller data set used by this analysis.

When this measurement is repeated using a larger data set a reduction in the overall uncertainty will be observed. Work has already commenced to use the larger 


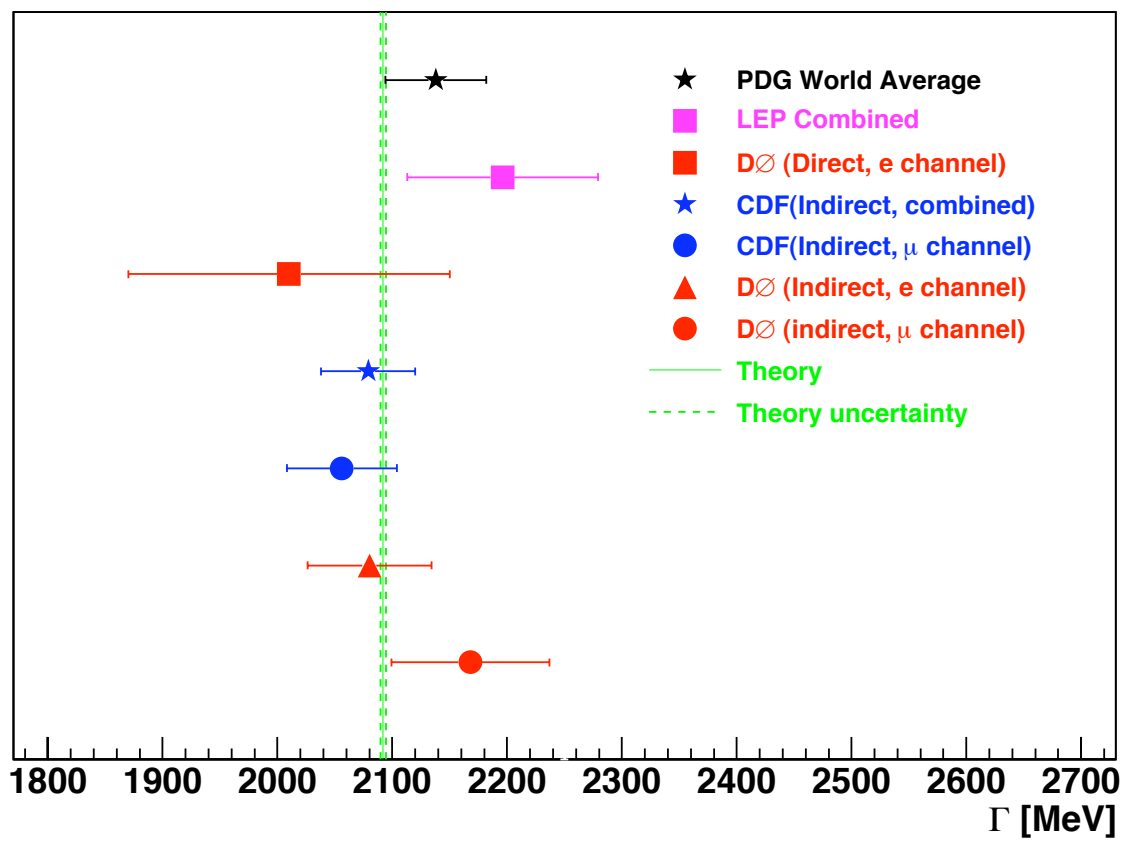

Figure 9.1: The Standard Model Prediction for the width of the W boson. The experimental measurements from DØ and CDF Run II are shown as points with error bars. The PDG average of all other experimental results is shown as a point with an error bar.

amounts of data to refine the detector simulation [75]. In addition to the improved statistical power there will be improvement in understanding of the systematic errors, such as a better understanding of the octant boundary structure, and improved theoretical tools, such as a replacement for the PYTHIA generator. 


\section{Chapter 10}

\section{Conclusions}

In this thesis the width of the $\mathrm{W}$ boson is determined using the indirect, ratio, method to be

$$
\Gamma_{W}=2168 \pm 22(\text { stat }) \pm 62(\text { syst })_{-16}^{+24}(\text { pdf }) \pm 4 \text { (other) } \mathrm{MeV}
$$

in agreement with the theoretical prediction [2] and other experimental measurements $[2,26,71-73]$. This provides another confirmation of the success of the Standard Model in describing the properties of fundamental particles.

The indirect method, described in Section 5.3, uses the ratio of the $\mathrm{W} \rightarrow \mu \nu$ to $\mathrm{Z} / \gamma^{*} \rightarrow \mu^{+} \mu^{-}$cross sections ${ }^{1}$. The determination of these cross sections are described in Chapter 6 and are interesting measurements in their own right. The cross sections have been calculated to NLO $[55,56]$ with small uncertainties, so they have been used as benchmarks, testing the performance of the $D \varnothing$ experiment and cross checking the determination of the luminosity. They have also been vital in understanding the detector, in particular the parts used to detect muons. One such example is the effect of the octant structure of the muon detectors, which was first studied in this analysis.

\footnotetext{
${ }^{1}$ The ratio actually uses the $\mathrm{Z} \rightarrow \mu^{+} \mu^{-}$cross section, which is obtained from the $\mathrm{Z} / \gamma^{*} \rightarrow \mu^{+} \mu^{-}$ cross section by a theoretical correction.
} 
The work performed to understand the detector has been used to improve the simulation of the $\mathrm{D} \varnothing$ detector. One example of this is given in Chapter 8, which describes the work done on the parameterised Monte Carlo simulation of the D $\varnothing$ detector, PMCS. This used $\mathrm{Z} / \gamma^{*} \rightarrow \mu^{+} \mu^{-}$events to model the resolution and efficiency of the central tracking system and the muon detectors.

The PMCs simulation has been used in physics analyses, some of which are described in Section 8.3. In particular there is a description of the determination of the efficiency of the $\mathrm{Z} / \gamma^{*} \rightarrow \mu^{+} \mu^{-}$event selection. As well as describing new techniques in modelling the detector resolution and efficiencies this thesis describes new procedures developed to estimate the uncertainty due to the choice of PDF in Chapter 7.

The new techniques will be used to repeat the measurements described in the thesis with the larger amounts of data now available. These new measurements will build upon the work performed in this thesis, which has resulted in the first measurement of the width of the $\mathrm{W}$ boson at DØ Run II using the indirect method in the muon channel. 


\section{Bibliography}

[1] F. Halzen and A.D. Martin, "Quarks and Leptons," Wiley (1984)

[2] S Eidelman et al, "Review of Particle Physics," Phys. Lett. B 592 1(2004)

[3] M.E.Peskin and D.V. Schroeder, "An Introduction to Quantum Field Theory," Perseus (1995)

[4] S. Gasiorowicz, "Quantum Physics (Second Edition)," Wiley (1996)

[5] J. Sedgbeer (editor), "Proceedings of the School for Young High Energy Physicists," RAL-TR-2003-07 (2003)

[6] J. Kroll, "Selected Results from UA1 at the CERN Sp̄̄S Collider," Presentation to UTEV (2004)

(http://www.fnal.gov/orgs/utev/talk-slides/2004_07_08_Joe_Kroll_UA1.pdf)

[7] S. Myers and E. Picasso, "The design, construction and commisioning of the CERN Large Electron Positron collider," Contemp. Phys 31387 (1990)

W. Schnell, "Design Study of a large electron-positron colliding beam-machine: LEP,” IEEE Trans. Nucl. Sci. 263130 (1979)

[8] N. Phinney, "SLC Final Performance and Lessons," http://arxiv.org/abs/physics/0010008 (2000) 
[9] G. Abbiendi et al [ALEPH, DELPHI, L3, OPAL and SLD Collaborations], "Precision Electroweak Measurements on the Z Resonance," Phys. Rept. 427 pp257-454 (2006)

[10] M.W. Gruenewald et al, "A Combination of Preliminary Electroweak Measurements and Constraints on the Standard Model," CERN-PH-EP/2004-069 (2004)

[11] G. Abbiendi et al [ALEPH, DELPHI, L3 and OPAL Collaborations], "Search for the Standard Model Higgs Boson at LEP," Phys. Lett. B 565 pp61-75 (2003)

[12] J. Thompson, "Introduction to colliding beams at Fermilab," FERMILAB-TM1909

[13] G. Jackson, "The Fermilab recycler ring technical design report. Rev. 1.2," FERMILAB-TM-1991

[14] S.Abachi et al [D $\varnothing$ Collaboration], "Observation of the Top Quark," Phys. Rev. Lett. 742632 (1995)

[15] F. Abe et al [CDF Collaboration], "Observation of Top Quark Production in p anti-p Collisions with the Collider Detector at Fermilab," Phys. Rev. Lett. 74, $2626(1995)$

[16] V. Abazov et al [CDF and DØ Collaborations], "Combination of CDF and D0 Results on W Boson Mass and Width," Phys.Rev. D70 092008 (2004)

[17] O. Bruning (editor), "LHC design report, Vol 1: The LHC main ring" CERN2004-003 (2004)

[18] O.B. Abdinov et al, "ATLAS Detector and Physics Performance Technical Design Report," CERN-LHCC-99-015 (1999)

[19] W. de Boer, "Grand Unified Theories and Supersymmetry in Particle Physics and Cosmology," Prog.Part.Nucl.Phys. 33 pp201-302 (1994) 
[20] R. D. McKeown and P. Vogel, "Neutrino Masses and Oscillations: Triumphs and Challenges," Phys.Rept. 394 pp315-356 (2004)

[21] V.M. Abazov et al [DØ Collaboration], "The Upgraded D $\varnothing$ Detector," Submitted to Nucl. Instrum. Methods A. (2005)

[22] V. D. Barger and R. J. N. Philips, "Collider Physics," Addison-Wesley Publishing (1987)

[23] G. Hesketh, "A Measurement of the Z Boson Production Cross Section Times

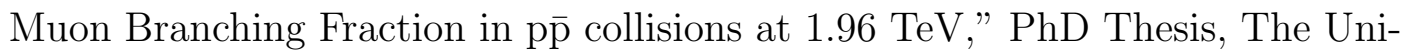
versity of Manchester (2003)

[24] C. Clément et al, "MuonID certification for p14," DØ Note 4350 (2004)

[25] D. Chapin et al, "Measurement of $\mathrm{Z} \rightarrow e^{+} e^{-}$and $\mathrm{W} \rightarrow e \nu$ Production Cross Sections Using One Tight Central Electron,” DØ Note 4897 (2005)

[26] J. Zhu, "Direct Measurement of the W Boson decay width in proton-antiproton collisions at $\sqrt{s}=1.96 \mathrm{TeV}, " \mathrm{PhD}$ Thesis, University of Maryland (2004)

[27] T. Sjöstrand et al, "Pythia v6.2 Physics and Manual," Computer Physics Commun. 135238 (2000)

[28] G. Corcella et al, "HERWIG 6.5: an event generator for Hadron Emission Reactions With Interfering Gluons (including supersymmetric processes)," JHEP 101238 (2001)

[29] S. Frixione and B.R. Webber, "Matching NLO QCD computations and parton shower simulations," JHEP 206029 (2002)

S. Frixione, P. Nason and B.R. Webber, "Matching NLO QCD and parton showers in heavy flavour production," JHEP 308007 (2003)

[30] Y. Fisyak and J Womersley, "D0gstar DØ GEANT Simulation of the Total Apparatus Response," DØ Note 3191 (1997) 
[31] CERn Application Software Group, “GEANT, Detector Description and Simulation Tool," CERN Program Library Long Writeups Q 123 (1993)

[32] G. Graham et al, "Status of the Fast Simulation, PMCS, December 2002," DØ Note 4059 (2002)

S. Eno et al, "Status of the Fast Simulation PMCS, v01-96-00," DØ Note 4097 (2003)

S. Eno and M. Verzocchi, "Status of the Fast Simulation Program PMCS, v0198-00," DØ Note 4165 (2003)

[33] P. Telford et al, "Retuning of pmcs_chprt to P13 and P14 data," DØ Note 4297 (2004)

[34] B. Zhou et al, "Parameterized MC Simulation (PMCS) muon package," D Note 4065 (2002)

[35] E. Nurse and P. Telford, "Measurement of the Cross section for Inclusive $Z$ Production in Di-muon Final States at $\sqrt{s}=1.96 \mathrm{TeV}, " \mathrm{D} \emptyset$ Note 4689 (2005)

[36] E. Nurse, "A Measurement of the Inclusive $\mathrm{Z} / \gamma^{*} \rightarrow \mu^{+} \mu^{-}$Cross Section and a Study of $\mathrm{W}$ and $\mathrm{Z}$ Events in $\mathrm{p} \overline{\mathrm{p}}$ Collisions at ", PhD Thesis, University of Manchester (2005)

[37] J.M. Butler, "Local Muon Momentum Resolution," DØ Note 4002 (2002)

[38] A. Askew, "Parametrizing the Local Muon Resolution in p13 Data for PMCS," DØ Note 4314 (2003)

[39] D. Chapin et al, " Measurement of $\mathrm{Z} \rightarrow e^{+} e^{-}$and $\mathrm{W} \rightarrow e \nu$ Production Cross Sections with $|\eta|<2.3$," DØ Note 4403 (2004)

[40] S.D. Ellis et al, "On Building Better Cone Jet Algorithms," eConf C010630 p513 (2001) 
[41] L. Feligioni and M. Narain, "Calibration of PMCS using fully simulated Technicolor signal in the $\rho_{T} \rightarrow W(\rightarrow e \nu)+\pi_{T}(\rightarrow b \bar{b}(\bar{c}))$ production channel for version p14 of DØ software," DØ Note 4966 (2005)

[42] P. Telford et al, "Measurement of $\sigma(\mathrm{p} \overline{\mathrm{p}} \rightarrow W \mathrm{X}) \times \operatorname{Br}(\mathrm{W} \rightarrow \mu \nu)$ at $\sqrt{s}=$ $1.96 \mathrm{TeV}, " \mathrm{D} \varnothing$ Note 4750 (2005)

[43] B. Abbott et al [DØ Collaboration], "Determination of the Mass of the W Boson Using the DØ Detector at the Tevatron," Phys. Rev. D 5812002 (1998)

[44] B. Tuchming, Private Communication (2006)

[45] J. Elmsheuser, "Search for the Higgs boson in $H \rightarrow W W^{(*)} \rightarrow \mu^{+} \nu_{\mu} \mu^{-} \overline{\nu_{\mu}}$ at DØ in Run II," DØ Note 4386 p16 (2004)

[46] T. Edwards et al, "Luminosity Constant for DØ Run II," DØ Note 4496 (2004)

[47] F. Abe et al [CDF Collaboration], "Measurement of the antiproton-proton total cross section at $\sqrt{s}=546$ and $1800 \mathrm{GeV}$," Phys. Rev. D 505550 (1994)

[48] C. Avila et al [E811 Collaboration], "A Measurement of the Proton-Antiproton Total Cross-Section at $\sqrt{s}=1.8$ TeV," Phys.Lett. B 445 pp419-422 (1999)

[49] S.Klimenko et al, "Averaging of the inelastic cross sections measured by the CDF and E811 experiments," Fermilab Note 0741 (2003)

[50] Y. L. Dokshitzer, "Calculation of the Structure Functions for Deep Inelastic Scattering and $e^{+}$and $e^{-}$Annihilation by Perturbation Theory in Quantum ChromoDynamics," Sov Phys. JETP 46 p641 (1977)

V. N. Gribov and L. N. Lipatov, "Deep Inelastic ep Scattering in Perturbation Theory," Sov. J. Nucl. Phys. 15 pp438, 675 (1972)

G. Altarelli and G. Parisi, "Asymptotic Freedom in Parton Language," Nucl. Phys. B 126298 (1977) 
[51] H.L. Lai et al, "Global QCD Analysis of Parton Structure of the Nucleon: CTEQ5 Parton Distributions," Eur.Phys. J. C 12 pp375-392 (2000)

[52] J. Pumplin et al, "New Generation of Parton Distributions with Uncertainties from Global QCD Analysis," JHEP 0207012 (2002)

[53] A.D. Martin et al, "MRST2001: partons and alpha_S from precise deep inelastic scattering and Tevatron jet data," Eur.Phys.J. C23 pp73-87 (2002)

[54] D. Stump et al, "Inclusive Jet Production, Parton Distributions, and the Search for New Physics," JHEP 0310 p046 (2003)

[55] T. Nunnemann, "NNLO Cross-Sections for Drell-Yan, $Z$ and $W$ Production using Modern Parton Distribution Functions," DØ Note 4476 (2004)

[56] R. Hamberg et al, "A Complete Calculation of the Order Alpha-S**2 Correction to the Drell-Yan K Factor," Nucl. Phys. B 359 pp343-405 (1991), Erratum-ibid. B 644 pp403-404 (2002)

[57] P. Telford, "The Pmcs_chprt Package," DØ Note 4923 (2005)

[58] P. Telford, "The Pmcs_muon Package," DØ Note 4935 (2005)

[59] U. Yang and Y. Kim, "ISR Studies on Drell-Yan," Fermilab MC Workshop (2003) (http://cepa.fnal.gov/patriot/mc4run2/MCTuning/031204/unki.pdf)

[60] R. Field, "Pythia Tune Set A," (2002) (http://www.phys.ufl.edu/r̃field/cdf/tunes/py_tuneA.html)

[61] B. Casey, Private Communication (2005)

[62] H. Schellman, "The longitudinal shape of the luminous region at D $\varnothing$," DØ Note $\mathbf{5 1 4 2}$ (2006) 
[63] H. L. Lai et al, "Improved Parton Distributions from Global Analysis of Recent Deep Inelastic Scattering and Inclusive Jet Data," Phys.Rev. D55 1280-1296 (1997)

[64] V. Abazov et al [DØ Collaboration], "Measurement of the $\mathrm{p} \overline{\mathrm{p}} \rightarrow W \gamma^{*}+\mathrm{X}$ cross section at sqrt(s) $=1.96 \mathrm{TeV}$ and $W W \gamma^{*}$ anomalous coupling limits," Phys.Rev. D 71091108 (2005)

[65] V. Abazov et al [DØ Collaboration], "Study of $Z \gamma$ events and limits on anomalous $Z Z \gamma$ and $Z \gamma \gamma$ couplings in ppbar collisions at $\operatorname{sqrt}(\mathrm{s})=1.96 \mathrm{TeV}$," Phys.Rev.Lett. 95051802 (2005)

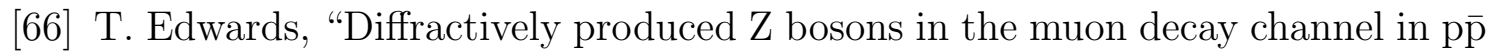
collisions at $\sqrt{s}=1.96 \mathrm{TeV}$, and the measurement of the D0 Run II Luminosity Monitor," PhD Thesis, University of Manchester (2006)

[67] B. E. Cox and J. R. Forshaw, "POMWIG : HERWIG for diffractive interactions," Comput.Phys.Commun. 144 104-110 (2002)

[68] V. Abazov et al [DØ Collaboration], " Search for Large Extra Spatial Dimensions in Dimuon Production with the D0 Detector," Phys. Rev. Lett. 95161602 (2005)

[69] T. Nunnemann, Private Communication (2006)

[70] W.K. Sakumoto, "W/Z Cross section Predictions for $\sqrt{s}=1.96 \mathrm{TeV}$," CDF Note 6341 (2003)

[71] J. Gardner, "Measurement of the W and Z Cross Sections in the Electron Channel and Extraction of the W Total Width from the Ratio," PhD Thesis, University of Kansas (2005) 
[72] D. Acosta et al [CDF collaboration], "First Measurements of Inclusive W and Z Cross Sections from Run II of the Fermilab Tevatron Collider," Phys. Rev. Lett. 94, 091803 (2005)

[73] A.V. Varganov, "The Production Cross Sections of the Weak Vector Bosons in Proton Antiproton Collisions at $\sqrt{s}=1.96-\mathrm{TeV}$ and a Measurement of the $\mathrm{W}$ Boson Decay Width," PhD Thesis, University of Michigan (2004)

[74] M. Grünewald et al, "The LEP Electroweak Working Group," lepewwg.web.cern.ch (2006)

[75] C. Jarvis, "p17 PMCS update for loose MUID and tracking," DØ Note 5076 (206)

[76] E. Nurse and P. Telford, "Measurement of the Cross section for Inclusive $Z$ Production in Di-muon Final States at $\sqrt{s}=1.96 \mathrm{TeV}$," DØ Note 4114 (2003) 


\section{Appendix A}

\section{Normalising Backgrounds}

A background can be normalised in two ways, the first is with respect to the signal and the second is with respect to the number of candidate events. The first method is used if the background, and its normalisation with respect to the signal, is understood. For instance this method is used in both analyses to determine the $\mathrm{Z} / \gamma^{*} \rightarrow \tau^{+} \tau^{-}$background. The second method is used when less is known about the background. For instance this method is used in both analyses to determine the background from cosmic rays.

\section{A.1 Normalising to the Signal}

Here it is known that the number of background events, $B$, is equivalent to some fraction, $f$, of the number of signal events, $S$. Therefore the total number of events, $N$, will be given by

$$
N=B+S
$$




$$
\begin{gathered}
N=f S+S \\
N=S(1+f) .
\end{gathered}
$$

Inverting this to get the number of signal events from the total number of events gives

$$
S=\frac{N}{1+f} .
$$

\section{A.2 Normalising to the Total Number of Events}

In this case it is known that the background represents a certain fraction of the total number of events. Thus

$$
N=f N+S,
$$

which can be rearranged to give the signal as a fraction of the total number of events

$$
S=N(1-f) .
$$




\section{Appendix B}

\section{Definition of the Octant Boundaries}

The muon chambers are divided into octants as can be seen in Figure 3.7. The definition of the octant boundaries is different in the FAmUS and the WAmUS. There are three regions

- In the octant boundary (region=2).

- On the edge of an octant boundary (region=1).

- Away from the octant boundary (region=0).

\section{B.1 The Central Region (The wamus)}

The octant boundaries in the central region are defined solely in terms of $\phi$. The size of the octant boundaries is treated separately for the 'diagonal' boundaries ${ }^{1}$

\footnotetext{
${ }^{1}$ These boundaries appear as diagonal lines when looked at in the $x y$ plane.
} 


\begin{tabular}{||l|l|l|}
\hline Region & 2 & 1 \\
\hline \hline Diagonal & 0.01 & 0.03 \\
Other & 0.01 & 0.02 \\
\hline \hline
\end{tabular}

Table B.1: Octant Boundaries in the Central Region. The table lists the distance, in $\phi$, from the edge of a given region to the centre of the octant boundary.

$\left(\phi_{\text {oct }}=\frac{\pi}{4}, \frac{3 \pi}{4}, \frac{5 \pi}{4}, \frac{7 \pi}{4}\right)$ and the other boundaries $\left(\phi_{\text {oct }}=0, \frac{\pi}{2}, \pi, \frac{3 \pi}{2}\right)$. Here $\phi_{\text {oct }}$ is the $\phi$ value at the centre of an octant boundary.

The diagonal boundaries are treated separately because the gaps between the muon chambers are larger in the diagonal boundaries. Table B.1 lists the size of the different regions in the central region. There are two rows, one for diagonal boundaries, one for non-diagonal ones.

The numbers describe the distance in $\phi$ between the centre of the octant boundary and the edge of the region. The first column gives these numbers for the first region, the second for the second region.

\section{B.2 The Forward Region (The FAmus)}

Again the diagonal and non diagonal boundaries are treated separately. This description will begin with the non diagonal boundaries. A muon is considered to lie within the horizontal boundary region (region $=2$ ) if $y$ is less than $3 \mathrm{~cm}$ and on the edge of the octant boundary region (region $=1$ ) if $y$ is between 3 and $5 \mathrm{~cm}$. A muon is considered to lie within the vertical boundary region (region $=2$ ) if $x$ is less than $3 \mathrm{~cm}$ and on the edge of the octant boundary region (region $=1$ ) if $x$ is between 3 and $5 \mathrm{~cm}$. The diagonal boundary regions are defined as diagonal strips by the following equations. 


$$
\begin{gathered}
\left(y-Y_{\text {width }}\right)<x<\left(y+Y_{\text {width }}\right) \\
\left(-y-Y_{\text {width }}\right)<x<\left(-y+Y_{\text {width }}\right)
\end{gathered}
$$

The in boundary region (region 2) has $Y_{\text {width }}=10 \mathrm{~cm}$ and the edge of the boundary region (region 1) has $Y_{\text {width }}=20 \mathrm{~cm}$. 


\section{Appendix $\mathrm{C}$}

\section{Old Form of the Drell Yan \\ Correction}

Section 9.1 describes the theoretical correction to obtain the $\mathrm{Z} \rightarrow \mu^{+} \mu^{-}$cross section from the $\mathrm{Z} / \gamma^{*} \rightarrow \mu^{+} \mu^{-}$cross section. The form of this correction was derived by the author to replace the old form which is described below.

The method of calculating the $\mathrm{Z} \rightarrow \mu^{+} \mu^{-}$cross section, used in Run I and early Run II results [76], was to evaluate the acceptance using $\mathrm{Z} \rightarrow \mu^{+} \mu^{-}$Monte Carlo and treat the photon exchange and interference terms purely as background. This is illustrated by

$$
\sigma_{\mathrm{Z} \rightarrow \mu^{+} \mu^{-}} \propto \frac{C_{D Y}}{\epsilon_{\mathrm{Z} \rightarrow \mu^{+} \mu^{-}}}
$$

where $\epsilon_{Z \rightarrow \mu^{+} \mu^{-}}$is the acceptance for $\mathrm{Z} \rightarrow \mu^{+} \mu^{-}$and $C_{D Y}$ is the fraction of the events, in the kinematic range chosen by the analysis, attributed to $\mathrm{Z} \rightarrow \mu^{+} \mu^{-}$. The factor $C_{D Y}$ is evaluated as follows 


$$
C_{D Y}=\frac{\sigma_{\mathrm{Z}} \rightarrow \mu^{+} \mu^{-} \epsilon_{\mathrm{Z}} \rightarrow \mu^{+} \mu^{-}}{\sigma_{\mathrm{Z} / \gamma^{*} \rightarrow \mu^{+} \mu^{-}} \mathrm{Z} / \gamma^{*} \rightarrow \mu^{+} \mu^{-}},
$$

where $\epsilon_{i}$ is the acceptance for process $i$ and $\sigma_{i}$ is a theoretical prediction for process $i$. Here process $i$ can be either $\mathrm{Z} \rightarrow \mu^{+} \mu^{-}$or $\mathrm{Z} / \gamma^{*} \rightarrow \mu^{+} \mu^{-}$. It can be seen that $C_{D Y}$ can be expressed as

$$
C_{D Y}=R_{\sigma} \frac{{ }^{\epsilon} \mathrm{Z} \rightarrow \mu^{+} \mu^{-}}{\epsilon_{\mathrm{Z} / \gamma^{*} \rightarrow \mu^{+} \mu^{-}}} .
$$

Substituting this into equation C.1 $\epsilon_{\mathrm{Z} \rightarrow \mu^{+} \mu^{-}}$cancels, leaving

$$
\sigma_{\mathrm{Z} \rightarrow \mu^{+} \mu^{-}} \propto \frac{R_{\sigma}}{\epsilon_{\mathrm{Z} / \gamma^{*} \rightarrow \mu^{+} \mu^{-}}}
$$

as in Section 9.1.

This method is no longer used as it requires the evaluation of the acceptance using both $\mathrm{Z} / \gamma^{*} \rightarrow \mu^{+} \mu^{-}$and $\mathrm{Z} \rightarrow \mu^{+} \mu^{-}$Monte Carlo and therefore requires the generation of twice as many events. The new approach has superseded this one as the standard form of correcting from the physical to $\mathrm{Z} \rightarrow \mu^{+} \mu^{-}$cross section at D $\varnothing$. 